\title{
\#26
}

APPRENDRE À GÉRER

DES COLLECTIONS

PATRIMONIALES

EN BIBLIOTHĖQUE

sous la direction de Dominique coq 


\section{Apprendre à gérer des collections patrimoniales en bibliothèque}

\section{Dominique Coq (dir.)}

DOI : 10.4000/books.pressesenssib. 643

Éditeur : Presses de l'enssib

Année d'édition : 2012

Date de mise en ligne : 4 avril 2017

Collection : La Boîte à outils

EAN électronique : 9782375460375

\section{QboOoks}

http://books.openedition.org

\section{Édition imprimée}

EAN (Édition imprimée) : 9791091281010

Nombre de pages : 180

\section{Référence électronique}

$\mathrm{COQ}$, Dominique (dir.). Apprendre à gérer des collections patrimoniales en bibliothèque. Nouvelle édition [en ligne]. Villeurbanne : Presses de l'enssib, 2012 (généré le 21 septembre 2021). Disponible sur Internet : <http://books.openedition.org/pressesenssib/643>. ISBN : 9782375460375 . DOI : https:// doi.org/10.4000/books.pressesenssib.643.

(c) Presses de l'enssib, 2012

Conditions d'utilisation :

http://www.openedition.org/6540 


$$
\text { \#20 }
$$

APPRENDRE À GÉRER DES COLLECTIONS PATRIMONIALES EN BIBLIOTHĖQUE

sous la direction de Dominique coq 


\section{APPRENDRE À GÉRER DES COLLECTIONS PATRIMONIALES EN BIBLIOTHĖQUE}


Titres disponibles

BA0 \#17 (2009)

\section{LA BÔ̂TE À OUTILS}

Favoriser la réussite des étudiants

sous la direction de Carine El Bekri-Dinoird

Les manuels de cette collection

BA0 \#18 (2009)

Mettre en œuvre un plan de classement

sous la direction de Bertrand Calenge

BA0 \#19 (2010)

Numériser et mettre en ligne

sous la direction de Thierry Claerr

et Isabelle Westeel

BA0 \#20 (2010)

Mettre en œuvre un service de questions-réponses en ligne

sous la direction de Claire Nguyen

BA0 \#21 (2010)

Communiquer! Les bibliothécaires,

les décideurs et les journalistes

sous la direction de Jean-Philippe Accart

BA0 \#22 (2011)

Mener l'enquête! Guide des études de publics

en bibliothèque

sous la direction de Christophe Evans

BAO \#23 (2011)

Créer des services innovants. Stratégies et

répertoire d'actions pour les bibliothèques

sous la direction de Marie-Christine Jacquinet

BAO \#24 (2011)

Mener un projet international: bibliothèques

françaises et coopération internationale

sous la direction de Raphaëlle Bats

BA0 \#25 (2012)

++++++++++++++++++++++

Développer la médiation documentaire numérique sous la direction de Xavier Galaup

PRESSES DE L'enssib

École nationale supérieure des

sciences de l'information et des

bibliothèques

17-21 boulevard du 11 novembre 1918

69623 Villeurbanne Cedex

A paraître

BA0 \#27 (2012)

Faire connaître et valoriser sa bibliothèque :

communiquer avec les publics

sous la direction de Jean-Marc Vidal 


\section{\#26}

\section{APPRENDRE À GÉRER DES COLLECTIONS PATRIMONIALES EN BIBLIOTHĖQUE}

Sous la direction de Dominique coq 
Ont contribué à cet ouvrage:

Noëlle Balley

Thierry Claerr

Gérard Cohen

Dominique Coq

Jocelyne Deschaux

Bernard Huchet
Carole Jacquet

Laure Jestaz

Patrick Lamotte

Fabienne Le Bars

Séverine Lepape

Florent Palluault

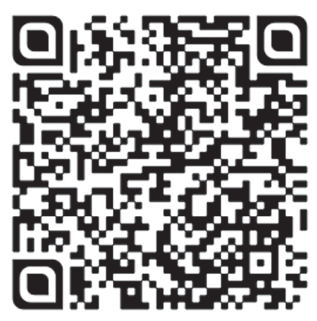

Une galerie d'images accompagne ce manuel. Pour y accéder, entrez l'URL suivante dans votre navigateur < http://www.enssib.fr/presses/galeriebao26 > ou scannez le QR code ci-contre avec votre mobile ou votre liseuse.

Apprendre à gérer des collections patrimoniales en bibliothèque [Texte imprimé]. / sous la direction de Dominique Coq. - Villeurbanne: Presses de l'enssib, cop. 2012. - 1 vol. (179 p.); 21 cm. - (La Boîte à outils; 26).

ISBN 979-10-91281-01-0. (br.): $22 €$

Dewey: 025.8

Rameau:

Bibliothèques - - Fonds spéciaux - - Livres rares - - Guides pratiques et mémentos

Patrimoine écrit - - Conservation et restauration - - France - - Guides pratiques et mémentos

Notice rédigée par la bibliothèque de l'enssib. 


\section{SOMMAIRE}

MODE D'EMPLOI

par Dominique Coq

p. 9

\section{PARTIE I. FACE À \\ UNE COLLECTION PATRIMONIALE}

p. 13

\section{LE DROIT DU PATRIMOINE}

\section{ÉCRIT : D'OŨ IL VIENT ?}

OU் IL VA?

par Gérard Cohen p. 14

LES FONDS PATRIMONIAUX : DES FONDS À HISTOIRES ... p. 14

UN DROIT COMPLIQUÉ POUR UNE HISTOIRE COMPLEXE . p. 17

LES CHARTES ÉTHIQUES

ET LES CODES

DÉONTOLOGIQUES

p. 23

$+++++++++++++++++++++++++++++++$

ENCADRÉS :

Quelques bibliothèques publiques avant 1789 p. 14

La Révolution et les bibliothèques

en 7 dates p. 15

De quoi l'État se mêle-t-il ? .... p. 19

Les fonds d'État p. 21
PATRIMOINE ET POLITIQUE DOCUMENTAIRE :

« LA BIBLIOTHĖQUE DANS SON ENTIER »

par Bernard Huchet p. 26

LE PATRIMOINE DES

BIBLIOTHĖQUES N'EST PAS

UNE TRADITION

PROFESSIONNELLE

p. 26

LE PATRIMOINE N'EST PAS UNE NOTION JURIDIQUE p. 27

LE PATRIMOINE DES

BIBLIOTHĖQUES NE

S'IDENTIFIE PAS

NÉCESSAIREMENT AUX

FONDS ANCIENS p. 28

LE PATRIMOINE EST D'ABORD AFFAIRE DE TRANSMISSION p. 30

LE PATRIMOINE DES BIBLIOTHĖQUES DOIT FAIRE L'OBJET D'UNE DÉCISION ... p. 31

LA POLITIQUE PATRIMONIALE REND NÉCESSAIRE LA COOPÉRATION p. 33

À QUOI SERT UNE RÉSERVE ET QU'Y METTRE ?

par Laure Jestaz p. 37

LA RÉSERVE EST UN LIEU . p. 37 
LA RÉSERVE, ESPACE MENTAL

DE CONSERVATION p. 39

CE QU'ON TROUVE DANS

UNE RÉSERVE

LES GRANDS PRINCIPES D'UNE RÉSERVE p. 43

$+++++++++++++++++++++++++++++++$

ENCADRÉ :

Les documents à mettre en réserve

p. 41

$+++++++++++++++++++++++++++++++$

COMMENT (BIEN) CONSERVER

LES COLLECTIONS

PATRIMONIALES ?

par Jocelyne Deschaux

LE PATRIMOINE ÉCRIT :

LES PRINCIPAUX MATÉRIAUX

ET LEUR CONSTITUTION .... p. 46

CONSERVATION DANS LES

MAGASINS

p. 47

LA CONSERVATION TOUT

AU LONG DE LA VIE EN

BIBLIOTHĖQUE

\section{ENCADRÉ :}

Les interventions en cas

d'inondation (et en phase

post-incendie)

p. 64
PARTIE II. DE LA COLLECTION À L'OBJET : IDENTIFIER ET SIGNALER LES DOCUMENTS

\section{LES LIVRES ANCIENS : FORMATS, CAHIERS, SIGNATURES, PAGE DE TITRE, FAUSSES ADRESSES, COLOPHON ET TOUTES CES SORTES DE CHOSES...} par Dominique Coq p. 70

IDENTIFIER L'ÉDITION p. 71

INDIVIDUALISER

L'EXEMPLAIRE p. 77

RELIURES FRANÇAISES SOIGNÉES ET COURANTES

(MI-XV ${ }^{\mathbf{e}}$ SIĖCLE - XIX ${ }^{\mathbf{e}}$

SIÈCLE) : ÉLÉMENTS

D'IDENTIFICATION

par Fabienne Le Bars p. 80

LEXIQUE p. 82

TYPOLOGIE DES RELIURES SOIGNÉES ET COURANTES p. 87

ESTAMPES : COMMENT IDENTIFIER LES TECHNIQUES ?

par Séverine Lepape p. 95

LES TROIS GRANDES

CATÉGORIES DE PROCÉDÉS p. 96 
$+++++++++++++++++++++++++++++++$

ENCADRÉ :

Données à signaler dans une notice d'estampe
INTEROPÉRABILITÉ p. 127

PRÉSENCE SUR LE WEB ET PERSPECTIVES D'ÉVOLUTION .... p. 129
COMMENT IDENTIFIER

LES PRINCIPALES TECHNIQUES PHOTOGRAPHIQUES?

par Patrick Lamotte

p. 107

TIRAGES POSITIFS PAPIER

LES PROCÉDÉS NÉGATIFS . p. 110

$+++++++++++++++++++++++++++++++$

ENCADRÉS :

Identification et support des négatifs monochromes p. 110

Principales altérations du support p. 114

\section{PARTIE III. GÉRER UN PROJET PATRIMONIAL}

p. 131

\section{METTRE À NIVEAU UNE BIBLIOTHĖQUE}

par Carole Jacquet p. 132

PAR OU் COMMENCER ?

DÉFINIR LES PHASES

DU CHANTIER

SIGNALER p. 133

REMETTRE EN ÉTAT p. 135

AMÉLIORER LES CONDITIONS DE CONSERVATION p. 138

VALORISER p. 139

\section{RÉVEILLER UN FONDS PATRIMONIAL}

par Noëlle Balley

p. 142

PERSPECTIVES

par Florent Palluault

QUELLES SONT LES OPTIONS

DE SIGNALEMENT ? p. 118

NORMALISATION DOCUMENTAIRE ET FORMATS INFORMATIQUES
UN FONDS CONSTRUIT PAR DES BIBLIOTHÉCAIRES ...... p. 142

REVISITER SON FONDS PATRIMONIAL p. 146

QUELQUES LEÇONS DICTÉES PAR L'EXPÉRIENCE p. 148 
INSCRIRE SON PROJET

DANS LES POLITIQUES

PATRIMONIALES NATIONALES

ET RÉGIONALES

par Thierry Claerr

p. 152

LES POLITIQUES

PATRIMONIALES AU

NIVEAU NATIONAL

p. 152

LES POLITIQUES

PATRIMONIALES AU NIVEAU

RÉGIONAL

QUELS SONT LES

PROGRAMMES NATIONAUX

DE SIGNALEMENT ?

QUELS SONT LES

PROGRAMMES NATIONAUX

DE NUMÉRISATION? p. 161

\section{MÉMENTO}

par Dominique Coq

p. 165

SIGLES ET ACRONYMES ... p. 169

GLOSSAIRE

p. 171

BIBLIOGRAPHIE

p. 175

LISTE DES

ILLUSTRATIONS

p. 177

LISTE DES AUTEURS

p. 179

+++++++++++++++++++++++++++++++++

ENCADRÉS :

Dispositifs spécifiques de

financements du signalement

des collections

Dispositifs spécifiques de

financements de la

numérisation

p. 164 


\section{MODE D'EMPLOI}

par Dominique Coq

«Vous venez d'être nommé-e responsable de la collection*1 patrimoniale d'une petite bibliothèque mais vous n'y connaissez pas grand-chose ou vos connaissances sont lointaines. D'où viennent ces vieux grimoires poussiéreux qui dorment sur les rayonnages? Qu'en faire ? A-t-on le droit de s'en débarrasser s'ils paraissent ne servir à personne ? Y en a-t-il qui sont rares et qu'il faudrait mettre en valeur? Quelques-uns sont catalogués mais c'est loin d'être le cas de tous, vous a dit votre prédécesseur. Comment faire? Et puis il y a de vieilles gravures dans des portefeuilles, des photographies anciennes ici et là. Comment savoir si elles ont quelque intérêt? Dans un coin des magasins, des moisissures sont brusquement apparues et semblent contaminer peu à peu les ouvrages. Comment s'en débarrasser ? Qui prévenir?

Si vous vous êtes mis-e dans cette situation délicate, ce manuel est pour vous. Vous y trouverez des conseils utiles, des recommandations de choses à faire et à ne pas faire, des listes de personnes ressources, d'opérations patrimoniales régionales et nationales qui pourraient vous aider à mettre en valeur votre fonds*, des témoignages de gens qui ont été dans votre cas et qui s'en sont pourtant sortis... »

Voilà ce que le prière d'insérer de ce volume pourrait dire de sa modeste ambition. Il ne s'agit pas, on l'aura compris, d'un nouvel ouvrage d'ensemble sur le patrimoine écrit, comme les remarquables traités dirigés par Raphaële Mouren ou Jean-Paul Oddos ${ }^{2}$ qui sont destinés à des personnes ayant une certaine familiarité avec le sujet. Ce manuel-ci se veut avant tout pratique, résumé, et s'adresse aux professionnels peu formés, confrontés pour la première fois à des collections patrimoniales dans de petites ou moyennes bibliothèques. Il veut leur donner quelques

1. Les termes suivis d'un astérisque (à leur première occurrence) sont définis dans le glossaire en fin d'ouvrage.

2. Raphaële Mouren (dir.), Manuel du patrimoine en bibliothèque, Paris, Éditions du Cercle de la Librairie, 2007. Jean-Paul Oddos (dir.), Le patrimoine, histoire, pratiques et perspectives, Paris, Éditions du Cercle de la Librairie, 1997. 
rudiments, quelques notions de base les aidant à appréhender ces collections, à les comprendre et à les gérer. Une sorte de guide pouvant être utilisé en accompagnement des stages de formation " afin de laisser plus de place aux travaux pratiques ", comme le préconisait très justement Hélène Richard dans un récent rapport de l'Inspection générale des bibliothèques ${ }^{3}(\mathrm{IGB})^{4}$.

\section{CE QUE VOUS TROUVEREל DANS CE MANUEL}

Les informations de ce manuel ont été organisées en trois étapes :

1. tout d'abord l'examen des collections patrimoniales en général et leur mise en perspective historique et juridique par Gérard Cohen qui précise leur statut, les règles domaniales qui les régissent et les devoirs qui s'imposent à vous en tant que responsable. S'agissant de la collection qui lui est confiée, c'est au responsable de décider, en concertation avec ses collègues de la bibliothèque, ce qui doit être conservé de manière définitive ; les réflexions de Bernard Huchet aideront à élaborer votre processus de "patrimonialisation ». Le même impératif de choix s'impose pour la constitution d'une réserve, trop souvent subie plutôt que choisie, comme le démontre Laure Jestaz. Enfin, la transmission d'une collection nécessite de la conserver dans de bonnes conditions ; les modes de préservation et de communication des documents patrimoniaux que recommande Jocelyne Deschaux sont issus d'une réflexion menée depuis de longues années par les professionnels, restaurateurs, conservateurs et historiens du livre ;

2. ensuite, un coup de zoom à l'intérieur de la collection fera découvrir divers types d'objets qui la composent : livres anciens, reliures, estampes, photographies. Une investigation

3. Hélène Richard, La formation aux questions patrimoniales dans les bibliothèques : rapport à $M$. le ministre de la Culture et de la Communication, Inspection générale des bibliothèques, septembre 2010 ( $n^{\circ}$ 2010-016), p. 44. [En ligne] < http://media.enseignementsup-recherche.gouv.fr/ file/2010/52/1/Formation_aux_questions_patrim_def_166521.pdf >.

4. Pour les sigles et acronymes, se reporter à la liste en fin d'ouvrage. 
archéologique, pour ainsi dire, au cours de laquelle divers spécialistes (Fabienne Le Bars, Séverine Lepape, Patrick Lamotte et moi-même) révéleront les procédés de fabrication de ces objets et les manières de les identifier pour les décrire. L'informatisation des notices descriptives nécessite des formes et des protocoles normalisés qui se sont imposés pour chaque type de document et dont il convient de suivre l'évolution. Ces enjeux et les perspectives futures du signalement sur le Web font l'objet de l'article de Florent Palluault ;

3. dans une dernière partie qui se voudrait plus " exemplative ", Carole Jacquet, du Centre culturel irlandais de Paris, et Noëlle Balley, de la Bibliothèque Cujas, feront part de leur expérience à propos d'opérations qu'elles ont menées récemment dans des bibliothèques, avec des moyens bien différents, pour réhabiliter et mettre à niveau des collections patrimoniales laissées depuis longtemps en sommeil. Chacune à sa manière, elles ont réussi à faire connaître leur fonds aux chercheurs de manière à ce qu'ils l'utilisent, ce qui est tout de même la fonction première d'une collection patrimoniale. Sans être parfaitement modélisables, ces retours d'expérience peuvent fournir quelques exemples à suivre, surtout si l'on sait utiliser les diverses aides scientifiques, techniques et financières qui existent au niveau régional et national, comme l'expose Thierry Claerr pour clore l'ouvrage.

\section{CE QU'IL NE FAUT PAS S'ATTENDRE À TROUVER DANS CE MANUEL}

Il n'a pas été question, ici, de traiter en abrégé l'ensemble des connaissances qu'il convient de bien posséder pour maîtriser ce domaine. Quantité d'aspects ne sont pas ou peu traités alors que certains trouveront que des sujets qui les intéressent moins font l'objet de trop longs développements.

Ainsi, on ne parlera guère d'acquisitions patrimoniales ni d'estimation pécuniaire de la collection ni de valeur d'assurance pour certaines pièces prêtées pour une exposition. Les problèmes posés par les dons et les legs 
dont pourra éventuellement bénéficier votre bibliothèque ne seront pas non plus évoqués. C'est de manière délibérée : ces questions viennent en second temps après que vous aurez pris la mesure de votre collection dans toutes ses composantes, même celles qui sont souvent négligées (je pense aux questions relatives à la "patrimonialisation ", ou au traitement des images fixes, par exemple, estampes et photographies).

D'une manière générale, ne vous attendez pas à trouver des solutions toutes faites pour chaque problème qui se posera à vous. Ce manuel est là pour vous donner un cadre, une aide pour vous orienter, et l'indication de quelques pistes, mais il ne remplacera pas la formation continue que vous devrez acquérir par vous-même.

Vous trouverez peu d'illustrations dans ce livre, comme c'est le cas dans tous les volumes de la collection "La Boîte à outils ». Cela peut être une gêne pour la compréhension des chapitres qui font appel à des reconnaissances visuelles comme l'identification des techniques des gravures, des matériaux de la reliure ou des procédés photographiques. Pour cela, reportez-vous à la galerie d'images en ligne ${ }^{5}$ qui accompagne ce manuel où vous trouverez un grand nombre d'illustrations. 


\section{PARTIE I}

\section{FACE À UNE \\ COLLECTION PATRIMONIALE}

1. LE DROIT DU PATRIMOINE ÉCRIT : D'OÙ IL VIENT ? OÙ IL VA?

par Gérard Cohen

$+++++++++++++++++++++++++++++++++++++++++++++$

2. PATRIMOINE ET POLITIQUE DOCUMENTAIRE :

« LA BIBLIOTHĖQUE DANS SON ENTIER »

par Bernard Huchet

3. À QUOI SERT UNE RÉSERVE ET QU'Y METTRE ?

par Laure Jestaz

++++++++++++++++++++++++++++++++++++++++++++++++++++

4. COMMENT (BIEN) CONSERVER LES COLLECTIONS PATRIMONIALES?

par Jocelyne Deschaux 
\begin{tabular}{|l|l|}
\hline LE DROIT DU PATRIMOINE ÉCRIT : & par Gérard Cohen \\
D'OU் IL VIENT ? OU் IL VA ? &
\end{tabular}

\section{LES FONDS PATRIMONIAUX : DES FONDS À HISTOIRES}

\section{L'ANCIEN RÉGIME}

Les fonds patrimoniaux des bibliothèques françaises reflètent l'histoire politique de notre pays. Jusqu'à la Révolution française ils se sont développés dans quatre domaines : le domaine royal, dont la Bibliothèque nationale de France $(\mathrm{BnF})$ est aujourd'hui l'héritière ; le domaine ecclésiastique, dont relevaient également les universités et les collèges ; le domaine municipal avec la constitution de bibliothèques publiques issues de dons ou de legs privés ; le domaine privé autour de grands patrimoines nobiliaires ou de riches particuliers ${ }^{1}$.

Dès le XvII ${ }^{\mathrm{e}}$ siècle, nombre d'entre elles sont ouvertes au public. L'origine de ces bibliothèques est diverse. Les bibliothèques municipales se constituent en général à partir du legs ou du don à la ville de la bibliothèque privée d'un particulier.

\section{ENCADRÉ 1 QUELQUES BIBLIOTHÈQUES PUBLIQUES AVANT 1789}

- Bibliothèques de Ville :

Abbeville (1643), Carpentras (1742), Chambéry (1783), Dijon (1764), Douai (1770), Dunkerque (1735), Grenoble (1772), Joigny (1746), La Rochelle (1783), Lyon (1731), Nemours (1754), Niort (1771), Oloron-Sainte-Marie (1764), Orléans (1715, propriété rétroactive en 1794 !), Paris, bibliothèque Moriau (1763), Reims (1764), Saint-Lô (1470), Salins-les-Bains (1593), Strasbourg (1765).

- Bibliothèques ecclésiastiques :

Annecy (1748), Besançon (1696), Castelnaudary (1774), Clermont-Ferrand (1742), Coutances (1751), Langres (1786), Meaux (ca. 1710), Mulhouse (1730), Nantes (1753), Perpignan (xviı ${ }^{\mathrm{e}}$ s.), Sens (1727), Toulouse (1772), Troyes (1651), Valence (1775).

1. Histoire des bibliothèques françaises, 4 vol., Paris, Promodis-Éd. du Cercle de la Librairie, 19881992. 
- Bibliothèques d'académies savantes :

Bordeaux (1740), Pau (1744).

- Bibliothèques princières :

Montbéliard (1765), Nancy (1750).

D’après : Patrimoine des bibliothèques de France, Paris, Payot, 1995.

\section{LA SAGA RÉVOLUTIONNAIRE}

La révolution de 1789 va, en plusieurs séquences, bouleverser ce paysage sans en faire totalement table rase puisque les grandes catégories antérieures se retrouvent in fine dans la nouvelle organisation : Bibliothèque nationale, bibliothèques municipales, bibliothèques savantes et d'université, bibliothèques privées.

\section{ENCADRÉ 2 LA RÉVOLUTION ET LES BIBLIOTHĖQUES EN 7 DATES}

- Loi du 2 novembre 1789 : confiscation des biens du clergé.

- Décret du 9 février 1792 : confiscation des biens des émigrés.

- Décret du 18 août 1792 : suppression des congrégations, universités, facultés, sociétés savantes.

- Décret du 8 mars 1793 : confiscation des biens des écoles, collèges, paroisses, communautés religieuses, universités.

- Décret du 27 janvier 1794 : création des bibliothèques de district (545) qui ont le droit exclusif de constituer leurs collections à partir des ouvrages confisqués, stockés dans les dépôts littéraires.
- Instruction du 7 mai 1796 : les écoles centrales, récemment créées dans les chefs-lieux de département, peuvent choisir pour leur bibliothèque les ouvrages utiles à l'enseignement dans les dépôts littéraires.

- Décret du 28 janvier 1803 : les bibliothèques des lycées, qui ont remplacé en 1802 les écoles centrales, sont « mises à la disposition et sous la surveillance » des municipalités où elles sont situées : c'est la naissance des bibliothèques municipales. 
On peut évaluer cet immense « butin » révolutionnaire à plusieurs millions de volumes, répartis à peu près pour moitié entre les bibliothèques municipales et les bibliothèques parisiennes, dont la Bibliothèque nationale. Mais en l'absence d'inventaires complets et agrégés, et compte tenu de notre méconnaissance des destructions opérées, tout chiffrage se doit de rester prudent.

\section{$\mathbf{X I X}^{\mathbf{e}}-\mathbf{x} \mathbf{x}^{\mathbf{e}}$ SIÈCLES : Le BOOM DES COLLECTIONS}

Les bibliothèques municipales vont désormais s'accroître de façon substantielle par achats, par libéralités des particuliers, par concessions et envois de l'État.

Les dons et legs des particuliers, surtout à partir des années 1830, sont tout à fait considérables : leur nombre avoisine celui des confiscations révolutionnaires en ordre de grandeur.

Les apports de l'État aux collections des bibliothèques sont tout aussi importants. Ils se font sous la forme de concessions ministérielles : l'État lance auprès de tel ou tel éditeur la commande d'une série d'ouvrages sur des sujets qui lui paraissent le mériter et en distribue les exemplaires aux bibliothèques. On estime, avec une relative précision, à 13 millions de volumes l'ensemble de ces concessions depuis 1809, date des premières d'entre elles, jusqu'au $x^{\mathrm{e}}$ siècle $^{2}$.

\section{UNE HISTOIRE À RÉPÉTITION : 1901-1905}

Une nouvelle vague de confiscations se produit au début $\mathrm{du} \mathrm{xx}^{\mathrm{e}}$ siècle : celles des bibliothèques des congrégations religieuses qui sont dissoutes par les lois de 1901-1905 sur la séparation de l'Église et de l'État ${ }^{3}$.

Seules les confiscations de 1901 méritent ce nom. Celles de 1905-1906 organisent principalement la récupération des confiscations révolutionnaires qui, par divers biais, étaient, tout au long du $\mathrm{XIX}^{\mathrm{e}}$ siècle, revenues à leurs propriétaires d'origine. Quelques bibliothèques de séminaires

2. Cf. Henri Comte, Les bibliothèques publiques en France, Paris, Presses de l'École nationale supérieure des bibliothèques, 1977, p. 72.

3. Isabelle Westeel, " Premiers jalons pour une histoire des confiscations de bibliothèques ecclésiastiques en 1905 », Revue française d'histoire du livre, n¹04-105, 1999, pp. 349-368. 
rejoignent néanmoins les collections publiques et trouvent le chemin de bibliothèques municipales, universitaires et d'archives départementales.

\section{UN DROIT COMPLIQUÉ POUR UNE HISTOIRE COMPLEXE}

\section{UNE FUSÉE JURIDIQUE À PLUSIEURS ÉTAGES}

Tout au long du XIX ${ }^{e}$ et du $\mathrm{XX}^{\mathrm{e}}$ siècle, un droit du patrimoine des bibliothèques se met en place et finit par entourer sa conservation et sa circulation. Un triple ensemble de lois et de règlements encadre ainsi la protection des fonds, repris dans divers codes.

\section{Les personnels}

Le premier ensemble organise l'affectation des personnels chargés de la conservation et de la mise en valeur des fonds patrimoniaux. Le statut général des fonctionnaires de l'État et des collectivités territoriales comporte quelques dispositions protectrices, notamment par l'obligation de contrôle des relations avec le secteur concurrentiel de droit privé (loi 84-16 du 11 janvier 1984, art. 72 et loi 93-122 du 29 janvier 1993) et en définissant les activités de droit privé incompatibles avec le statut des fonctionnaires en retraite ou en disponibilité, en l'occurrence conservateurs ou responsables de conservation (loi 84-16 du 11 janvier 1984, art. 72 et décret 95-168 du 17 février 1995). Les statuts des professions de conservation (conservateurs et bibliothécaires) leur attribuent une mission patrimoniale (décret 92-26 du 9 janvier 1992 et décret 91-841 du 2 septembre 1991) sans que ce patrimoine soit défini.

\section{Les collections}

Le deuxième ensemble fixe le cadre légal qui régit la conservation et la protection des fonds. Concernant les bibliothèques municipales, le Code du patrimoine, partie législative (L. 310-1 à 6) et réglementaire (R. 310-1 à 14) prévoit un certain nombre de dispositions protectrices, qui se rattachent principalement au mode de contrôle exercé par l'État en fonction du type de bibliothèque (la richesse des collections influant sur cette classification) et aux types de contrôles exercés par l'État sur les fonds 
patrimoniaux (contrôle technique, vols, échanges, transferts, restaurations, communications hors site, désaffectations).

\section{Le contrôle des biens culturels}

Le troisième ensemble s'attache au contrôle de la circulation et de la restitution des biens culturels, ensemble de biens patrimoniaux visés dans une nomenclature (Code du patrimoine, partie législative, L. 111-1 à 7 et réglementaire, art. R. 111-112). Ces dispositions encadrent l'exportation (hors des frontières douanières de l'Union européenne), que celle-ci soit définitive ou temporaire, la sortie (à l'intérieur des frontières douanières de l'Union européenne), que celle-ci encore soit temporaire ou définitive, la sortie et l'exportation illicites, enfin le statut de Trésor national de certains de ces biens. Elles fixent également les modalités de restitution en cas de délit et les instances chargées de les faire appliquer.

Ce droit s'applique aux fonds d'État comme à ceux qui sont propriété des collectivités. Il est erroné de croire que seuls les premiers seraient soumis à ces dispositions et que les autres en seraient exemptés. Encore aujourd'hui le préfet de département et le préfet de région interviennent dans leur gestion à des titres divers. Les lois de décentralisation de 1982 n'entraînent pas de modification du dispositif d'ensemble. En revanche, l'État relâche progressivement la pression directe que les textes lui autorisaient à exercer. Le contrôle technique rempli par l'IGB existe toujours et les conservateurs d'État, dont la mise à disposition auprès des communes est encadrée par des conventions ville-État depuis 2010, continuent de veiller à leur conservation et à leur mise en valeur. Mais l'antinomie née de l'autonomie des villes (et des bibliothèques qui en sont un des services) et de la tutelle résiduelle de l'État ouvre indéniablement sur une clarification à venir. 


\section{ENCADRÉ 3 DE QUOI L'ÉTAT SE MÊLE-T-IL ?}

- contrôle technique sur les bibliothèques (Inspection générale des bibliothèques) ;

- affectation de conservateurs d'État auprès des bibliothèques municipales classées par convention ville-État ;

- affectation des exemplaires de dépôt légal éditeur (2) à la $\mathrm{BnF}$ et imprimeur (1) aux bibliothèques attributaires ;

- contrôle de la circulation des biens culturels des bibliothèques par le Service du livre et de la lecture (SLL) à l'intérieur et à l'extérieur de l'Union européenne ;
- contrôle des opérations de restauration de documents et d'objets patrimoniaux par le préfet de région (Direction régionale des affaires culturelles, DRAC) sur avis du Comité technique restauration $(\mathrm{CTR})^{* 4}$;

- contrôle de la sûreté et des échanges de documents et d'objets patrimoniaux entre bibliothèques par le préfet de département.

4. Voir l'article de Jocelyne Deschaux : « Comment (bien) conserver les collections patrimoniales ? », particulièrement pp. 65-67.

\section{LE DÉPÔT LÉGAL : UNE OBLIGATION IRRÉGULIÈRE}

Le dépôt légal est actuellement régi par le titre III du livre $\mathrm{I}^{\mathrm{er}} \mathrm{du}$ Code du patrimoine. La BnF est affectataire au titre du dépôt légal d'éditeur de 2 exemplaires des imprimés (livres, périodiques, cartes, gravures), des médailles, des phonogrammes, des vidéogrammes et des « œuvres informatiques » (logiciels, bases de données, sites Internet.) Le Centre national du cinéma est affectataire du dépôt légal des œuvres cinématographiques. L'Institut national de l'audiovisuel reçoit celui des programmes de radio et de télévision. Le dépôt légal d'imprimeur en 1 exemplaire (imprimés seulement) est affecté à une liste de bibliothèques régionales attributaires par décret.

Le dépôt légal a considérablement étendu le champ du domaine patrimonial (au sens légal, c'est-à-dire de ce qui relève du patrimoine de la nation). Il a cependant été loin d'être toujours exhaustif. Il présente, tant à la $\mathrm{BnF}$ que dans les bibliothèques municipales attributaires, des lacunes considérables qu'il est nécessaire de combler afin de disposer de séries cohérentes. 


\section{LE DROIT DOMANIAL : UN MONARQUE MAINTES FOIS DÉPOSSÉDÉ 5}

Inaliénabilité, imprescriptibilité de la propriété publique (et des actions en recouvrement mais pas des délits qui leur sont liés !) : ces deux termes magiques surplombent le droit de propriété du patrimoine des bibliothèques en tant que relève du domaine public des personnes publiques qui le détiennent. Comme l'histoire le montre, ce droit a été long à s'établir, une fois établi il a été maintes fois violé ou contourné et il a récemment changé de face.

À la fin de l'Ancien Régime, le domaine public se restreint au domaine royal $^{6}$. Son inaliénabilité ne s'applique pas aux objets mobiliers dont les livres et objets de bibliothèque font partie. Les livres et manuscrits de la Bibliothèque royale sont volontiers prêtés, donnés, vendus ou échangés. La notion même de patrimoine culturel n'existe pas : elle apparaît, réduite au patrimoine monumental, autour des années 1830, au sein d'un mouvement romantique passionné d'histoire et révulsé par le vandalisme issu de la Révolution.

Les deux vagues de confiscation s'étant effectuées au profit de la nation, elle en détient la propriété et en exerce les droits dans le cadre d'un droit de la propriété publique qui se renforce tout au long des $\mathrm{XIX}^{\mathrm{e}}$ et $\mathrm{xx}^{\mathrm{e}}$ siècles. Néanmoins ces fonds, propriété de l'État, dont une grande partie se retrouve dans les bibliothèques municipales où ils ne sont qu'en dépôt, ne bénéficient pas d'une protection jugée suffisante jusqu'à ce que l'ordonnance royale de 1839 interdise l'aliénation des collections par les villes, fonds d'État compris. De la même façon, les concessions ministérielles, dont nous avons vu l'ampleur, ne reçoivent le statut définitif de

5. Henri Comte, op. cit., pp. 127-167. Jean-Gabriel Sorbara, " De quelques conséquences du Code général de la propriété des personnes publiques sur la gestion des collections », Bulletin des bibliothèques de France, 2009, t. 54, n 1, pp. 38-40. [En ligne] < http://bbf.enssib.fr/consulter/ bbf-2009-01-0038-005 >. Raphaële Mouren (dir.), Manuel du patrimoine en bibliothèque, Paris, Éditions du Cercle de la Librairie, 2007, pp. 31-37.

6. Albert Rigaudière, Pouvoir et institutions dans la France médiévale. II, des temps féodaux au temps de l'État, Paris, A. Colin, 1994. La première apparition de l'ancêtre du domaine public se retrouve dans une proclamation de l'assemblée de Vincennes (1329) puis, de manière plus formelle, dans l'édit de Moulins (février 1566). 
fonds d'État que par décret du $1^{\text {er }}$ juillet 1897, au terme d'une incertitude juridique qui aura duré tout le $\mathrm{xIX}^{\mathrm{e}}$ siècle ${ }^{7}$.

\section{ENCADRÉ 4 LES FONDS D'ÉTAT}

- les fonds issus des deux confiscations (Révolution et 1901-1905) et des prises de guerre ;

- des exemplaires du dépôt légal ;

- les concessions ministérielles ;
- les dations* reçues en paiement de certaines obligations fiscales;

- les acquisitions de l'État et de ses établissements publics, quel qu'en soit le mode.

Les collections de livres acquises par les communes relèvent de leur propriété. Néanmoins, l'État y exerce un droit de regard : en tant que dépendantes du domaine public mobilier de la nation, il en interdit alors l'aliénation, comme il interdit celle des fonds des bibliothèques municipales qui n'ont pas été confisqués en 1789. Là encore, les bibliothécaires, faisant fi du droit, ne se sont pas privés de désherber. La fragilité de ce droit prétendu régalien éclate encore dans la confusion qui règne dans les bibliothèques entre les fonds d'État qu'elles détiennent et leurs autres collections. Aucune marque de propriété par l'État ne figure sur les exemplaires et ce n'est que par leur ancienneté qu'on peut en déduire leur statut.

\section{PATRIMONIALITÉ : CHANGEMENT DE CAP DEPUIS 2006}

Les bibliothèques de l'État et des collectivités publiques relèvent actuellement du droit des personnes publiques. La domanialité des biens mobiliers que possèdent l'État et les collectivités et dont les documents de bibliothèques font partie, peut être soit publique soit privée. Jusqu'en 2006, elle a été dite publique lorsque le bien était affecté à l'usage d'un public ou à un service public. Elle était privée dans tous les autres cas. Relevaient

7. Nombre de textes, dont un arrêté du 25 juillet 1838, utilisaient le verbe « donner » pour qualifier ces concessions. 
ainsi de la domanialité publique* non seulement les fonds d'État mais les collections acquises par les collectivités publiques à l'usage du public. Les fonds patrimoniaux figurant dans les bibliothèques municipales constituées avant la Révolution mais qui n'ont fait l'objet d'aucune mesure de confiscation relevaient également du domaine public.

Les choses ont changé. En 2006, le législateur introduit à l'article 2112-1 du Code général de la propriété des personnes publiques (dit CG3P) une disposition tout à fait novatrice :

Font partie du domaine public mobilier de la personne publique qui en est propriétaire les biens présentant un intérêt public du point de vue de l'histoire, de l'art, de l'archéologie, de la science ou de la technique et, notamment, en ce qui concerne les bibliothèques :

- un exemplaire identifié de chacun des documents dont le dépôt est prescrit aux fins de constitution d'une mémoire nationale par l'article L. 131-2 du Code du patrimoine ;

- les objets mobiliers classés ou inscrits au titre du chapitre 2 du titre II du livre VI du Code du patrimoine ou situés dans un immeuble classé ou inscrit et concourant à la présentation au public de parties classées ou inscrites dudit immeuble (L. 2112-1, 6 ) ;

- les objets mobiliers autres que ceux mentionnés au $6^{\circ}$ cidessus, présentant un intérêt historique ou artistique, devenus ou demeurés propriété publique en application de la loi du 9 décembre 1905 concernant la séparation des Églises et de l'État (L. 2112-1, $7^{\circ}$ ) ;

- les collections de documents anciens, rares ou précieux des bibliothèques (L. 2112-1, 10).

Se pose néanmoins la question du statut juridique des nouveaux objets, numériques et incorporels, notamment réalisés à partir des fonds patrimoniaux, lesquels constituent un nouveau domaine public immatériel que le législateur n'a pas encore défini concernant les bibliothèques.

Il faut ainsi désormais qu'un document ou un objet de bibliothèque présente un intérêt public du point de vue de l'histoire, de l'art, de l'archéologie, de la science ou de la technique ou entre dans la nomenclature, d'ailleurs non exhaustive, figurant dans l'article. On est passé d'une 
patrimonialité subie à une patrimonialité active : en d'autres termes, c'est aux bibliothécaires de décider si un document ou un objet est ancien, rare, précieux ou non, et s'il présente un intérêt historique, artistique, archéologique, scientifique ou technique.

Pour que leur décision ne soit pas subjective ni fluctuante, deux conditions doivent être réunies. Elle doit d'abord être collective : une décision solitaire présente les plus grands risques. Elle doit ensuite être scientifique et reposer sur les consensus de la communauté professionnelle des bibliothécaires, éclairés, si besoin est, par ceux de la communauté des historiens, archéologues, scientifiques ou techniciens spécialistes du domaine sur lequel porte le document, l'objet ou le fonds. Dans les mêmes conditions, celui-ci peut être dépatrimonialisé, disposition qui ouvre la voie à un désherbage raisonné des fonds patrimoniaux.

\section{LES CHARTES ÉTHIQUES ET LES CODES DÉONTOLOGIQUES}

L'intensification des échanges de biens et d'informations entre les nations, l'abaissement des frontières, l'enchérissement des biens patrimoniaux, l'intrication de plus en plus étroite du secteur public et du secteur privé en matière culturelle, avec, en particulier, le développement d'industries culturelles puissantes, l'amplification des revendications mémorielles, bien d'autres facteurs encore, appellent d'autres réponses que la simple réponse réglementaire. C'est pourquoi l'éthique et la déontologie viennent en renfort des dispositifs classiques en impliquant directement la responsabilité professionnelle des individus et des institutions.

La déontologie, dans tous les métiers, prend aujourd'hui une importance croissante. Comme l'étymologie l'indique, elle désigne l'ensemble des devoirs qui incombent au praticien d'un métier ou d'une profession au regard de situations où un conflit, notamment d'intérêt, peut surgir entre sa mission et les conditions dans lesquelles il la remplit. « Devoir » n'a pas ici forcément un sens moral : c'est l'ensemble des bonnes pratiques qu'un métier ou une profession juge indispensables à la réalisation des standards qu'il s'est fixés. La notion de standard se distingue elle-même de celle de norme comme la déontologie se distingue de la législation : c'est 
une exigence qu'on se donne, souvent collectivement, non une obligation à laquelle on se soumet.

La déontologie est particulièrement adaptée au règlement des situations qui n'entrent pas dans le cadre prévu par la loi ou qui appellent une interprétation de celle-ci, souvent dans un sens plus rigoureux. Il est donc naturel que les responsables de fonds patrimoniaux de grande valeur, une valeur qui peut être vénale mais aussi scientifique, religieuse, idéologique, affective, identitaire, etc., éprouvent le besoin d'une déontologie professionnelle. Celle-ci n'a pas toujours reçu en France, dans les bibliothèques, l'accueil qui lui a été réservé dans d'autres pays. L' International Federation of Library Associations and Institutions (IFLA) recense néanmoins des codes déontologiques du bibliothécaire dans une trentaine de nations ${ }^{8}$. Comme cette approche est avant tout professionnelle, c'est aux associations professionnelles de s'en saisir. C'est pourquoi l'Association des bibliothécaires de France ( $\mathrm{ABF}$ ) et l'Association des professionnels de l'information et de la documentation (ADBS) se sont dotées d'un code de déontologie pour l'une en 2003, pour l'autre en adhérant au Code déontologique de l'European Council of Information Associations (ECIA) en 2005. Les institutions chargées de conserver des ensembles patrimoniaux sont confrontées aux mêmes dilemmes. D'où la multiplication des chartes éthiques qui fixent des engagements visant à impliquer aussi bien leur administration que leurs usagers et partenaires extérieurs. Dès la fin des années 1990, de grandes institutions patrimoniales se sont dotées de chartes éthiques, encadrant certaines de leurs activités particulièrement exposées.

Le musée du Louvre, précurseur dans le secteur patrimonial, a édicté ou signé plusieurs chartes éthiques : dans le domaine du mécénat (charte éthique du musée du Louvre en matière de mécénat, 2003) et des dons privés (charte éthique du musée du Louvre pour ses relations avec les donateurs individuels), dans le domaine du développement durable (charte Développement durable des Établissements publics et Entreprises publiques, 2010).

8. < http://www.ifla.org/en/faife/professional-codes-of-ethics-for-librarians >. 
La BnF à son tour a présenté une charte éthique pour ses relations avec ses mécènes en 2009. On peut citer aussi nombre de bibliothèques municipales et universitaires qui se sont dotées de chartes mais où l'accent est plutôt mis sur la constitution des collections que sur leur patrimonialité, malgré les contraintes spécifiques que celle-ci fait peser sur ceux qui en sont responsables comme sur les institutions qui les conservent.

Enfin, le Service du livre et de la lecture au ministère de la Culture et de la Communication (MCC) élabore actuellement une charte de la conservation destinée à fixer les bonnes pratiques en matière de conservation telles que la réflexion théorique en la matière ainsi que l'évolution des méthodes au niveau international les ont répandues depuis plusieurs années.

Il est important de bien distinguer les chartes éthiques et les codes déontologiques : les chartes éthiques énoncent en général ce que telle ou telle institution s'interdit de faire dans telle ou telle circonstance ; les codes déontologiques codifient un ensemble de bonnes pratiques correspondant aux standards professionnels et "vertueux » d'un métier. À la différence des chartes éthiques, les codes déontologiques peuvent comporter un processus juridictionnel et disciplinaire, et notamment être assortis de sanctions pour les contrevenants pouvant aller jusqu'à une exclusion temporaire ou définitive de la communauté professionnelle ${ }^{9}$.

Concluons brièvement. Le patrimoine de nos bibliothèques est exceptionnel. Aussi, chaque fois qu'un bibliothécaire rencontre une pièce de cet ensemble, doit-il être conscient qu'il ne doit pas agir sans connaître sa forme et son contenu, son histoire, le droit qui la protège, les obligations qu'il a vis-à-vis de l'institution qui la conserve et les devoirs personnels qu'elle exige de lui.

9. Voir le code déontologique des bibliothécaires britanniques : The Library Association: code of professionnal conduct, art. 2, k. < http://archive.ifla.org/faife/ethics/lacode.htm >. 


\section{2}

PATRIMOINE ET POLITIQUE DOCUMENTAIRE : « LA BIBLIOTHĖQUE DANS SON ENTIER 》

L'historique des collections des bibliothèques fait apparaître certains caractères de leur légitimité, tout en éclairant les motifs d'un statut juridique épineux ; mais il ne résout pas la question capitale qu'il faut aborder maintenant : puisqu'il est convenu que certaines bibliothèques au moins soient détentrices d'un "patrimoine », quel est véritablement son périmètre au sein de chaque établissement ? Comment lui donner une dynamique et l'articuler avec les autres notions qui structurent la politique documentaire, le souci d'analyse des publics par exemple, ou le respect des règles domaniales ? Pour diverses raisons qui tiennent à l'évolution de notre culture professionnelle, la notion même de « bibliothèque patrimoniale » et de patrimoine des bibliothèques prête à confusion : chacun donne à ce terme un sens qui fausse le débat, se fondant sur d'incertains critères d'histoire ou de réglementation. Quelques éclaircissements préalables sont donc nécessaires pour fonder la proposition qui va suivre.

\section{LE PATRIMOINE DES BIBLIOTHĖQUES N'EST PAS UNE TRADITION PROFESSIONNELLE}

Comme le rappelait Jean-Luc Gautier-Gentès en $2009^{1}$, le terme de « patrimoine " appliqué à certaines collections des bibliothèques publiques est l'héritage du rapport Desgraves, présenté au directeur du livre en 1982. C'est un choix sémantique seulement trentenaire, et dont l'objectif de circonstance était alors de faire bénéficier les bibliothèques de crédits spécifiques, après le rendez-vous manqué de l'Année du patrimoine en 1980.

1. Jean-Luc Gautier-Gentès, "Le patrimoine des bibliothèques : rapport à Monsieur le directeur du livre et de la lecture », Bulletin des bibliothèques de France, 2009, t. 54, n 3. [En ligne] < http://bbf.enssib.fr/consulter/bbf-2009-03-0027-010 >. 
Le rapide succès qu'a rencontré cette appellation dans le discours professionnel et dans les organigrammes des bibliothèques prouve néanmoins qu'elle y était nécessaire pour désigner, codifier et promouvoir une activité jusque-là pratiquée de manière empirique dans les établissements, dépassant par sa nature et ses ambitions les simples opérations de conservation matérielle des collections anciennes. Il s'agissait bien de nommer une politique prospective impliquant l'existence d'une théorie fondatrice et la définition d'objectifs à court ou moyen terme, de même que le besoin de progresser par la confrontation d'idées, l'action collective et l'évaluation régulière des travaux accomplis.

Le rapport Desgraves soulignait à bon droit dans son introduction que " traiter du patrimoine des bibliothèques, c'est traiter des bibliothèques dans leur entier ${ }^{2}$, et qu'une forme de vigilance patrimoniale devait s'exercer également sur les collections courantes. Malheureusement, la commission n'avait pu réellement développer ce point, recentrant son propos sur les « fonds anciens, rares et précieux » dont la mise en avant lui paraissait prioritaire, pour des raisons de sauvegarde notamment.

\section{LE PATRIMOINE DES BIBLIOTHĖQUES N'EST PAS UNE NOTION JURIDIQUE}

Il est notable qu'aucun des textes réglementaires qui régissent les bibliothèques ne cite explicitement leur patrimoine : le silence des lois ne saurait surprendre, puisque les professionnels se sont, en somme, emparés d'un terme que se réservaient jusque-là d'autres services de conservation, notamment ceux dont l'activité s'exerce en faveur du patrimoine monumental. On a vu ci-dessus que depuis 2006, la nouvelle définition du domaine public mentionne « les collections de documents anciens, rares ou précieux des bibliothèques ${ }^{3}$, mais non leurs collections patrimoniales, suivant la périphrase issue du rapport Desgraves et retenue en 1988 pour

2. Louis Desgraves, Le patrimoine des bibliothèques : rapport à Monsieur le Directeur du Livre et de la lecture, 1982.

3. Code général de la propriété des personnes publiques, article L.2112-1, $10^{\circ}$. Cf. ici même les commentaires qu'en donne Gérard Cohen, « Le droit du patrimoine écrit : d'où il vient ? Où il va ? », p. 22. 
définir les modalités du contrôle technique de l'État*. Certes, l'insertion ${ }^{4}$ dans le récent Code du patrimoine de ces dernières dispositions, qui relevaient jusque-là du Code général des collectivités territoriales, est à considérer comme une reconnaissance légale du rôle patrimonial de nos établissements : mais elle s'est effectuée à droit constant, et n'apporte aucune information nouvelle sur la définition d'un patrimoine qui leur serait spécifique.

Il en résulte que les bibliothécaires n'ont pas d'obligation légale ni réglementaire fondée sur la notion de patrimoine, puisque celle-ci n'est pas explicitement formulée par le législateur. Force nous est donc de considérer que le terme est vacant, c'est-à-dire qu'il peut être librement défini lors de l'élaboration d'une politique documentaire. À la différence du domaine public dont l'appartenance est intrinsèque aux documents qui le composent, la construction du patrimoine sera donc facilement soumise à des critères d'opportunité qui varient suivant les prescriptions documentaires de chaque établissement. Il convient toutefois d'examiner quels rapports peuvent ou doivent s'établir entre patrimoine et domaine public, pour déterminer le degré d'inaliénabilité des collections patrimoniales.

\section{LE PATRIMOINE DES BIBLIOTHĖQUES NE S'IDENTIFIE PAS NÉCESSAIREMENT AUX FONDS ANCIENS}

On ne saurait construire une dynamique du patrimoine sur un critère strictement chronologique, comme on croyait encore pouvoir le faire à la fin du $\mathrm{xx}^{\mathrm{e}}$ siècle : ainsi, les commentaires de l'article 8 de la Charte des bibliothèques ${ }^{5}$, publiée sans réelle portée réglementaire par le Conseil supérieur des bibliothèques en 1991, font entrer dans les collections patrimoniales " tout document de plus de cent ans d'âge ». Les inconvénients que provoque une telle formule sont assez connus : sacralisation abusive

4. Par le décret $n^{\circ}$ 2011-574 du 24 mai 2011. La circulaire d'application de ces mesures, produite par la Direction du livre et de la lecture en 1988, est aujourd'hui largement dépassée, mais n'a pas été remplacée par un texte officiel plus récent.

5. Document téléchargeable en ligne, par exemple sur le site bibliofrance.org : < http://www. bibliofrance.org/ressources_professionelles/index.php?option=com_jdownloads\&Itemid= 2\&task=view.download\&cid=18 > (consulté en mars 2012). 
de collections anciennes sans la moindre valeur scientifique, conservation coûteuse et défaillante, par défaut de moyens proportionnés à leur nombre, d'ouvrages sans rapport avec une quelconque orientation documentaire des fonds, difficultés à maîtriser dans sa globalité le spectre disciplinaire formé par leurs contenus. Il ne suffit évidemment pas qu'un ouvrage soit devenu centenaire pour garantir le besoin de sa conservation dans les fonds de l'établissement.

C'est en termes de cohérence globale dans la détermination d'une politique documentaire qu'il faut être particulièrement critique à l'égard d'une césure de ce type. Dans son ouvrage sur Les politiques d'acquisition, Bertrand Calenge recommande ainsi de procéder à des éliminations dans les collections " semi-patrimoniales " avant qu'elles aient franchi le seuil fatidique des cent ans ${ }^{6}$ : cette borne est à la fois trop lointaine pour éviter qu'entre-temps s'entassent dans les magasins des collections dépourvues d'intérêt qu'on laissera toujours le soin de classer à d'hypothétiques successeurs, et trop proche pour qu'on puisse vraiment se prévaloir d'un recul suffisant pour opérer dans leur masse des choix scientifiques pertinents. Il en résultera donc d'inévitables distorsions entre les positions de principe et leur application : procéder après-coup à une "patrimonialisation » des collections courantes, parce qu'on établit une limite arbitraire entre patrimoine et collections contemporaines, condamne à fonder le patrimoine de l'établissement sur le régime imparfait du constat, quand il faudrait pratiquer la prescription.

Par ailleurs, une vision du patrimoine qui s'articule de manière préférentielle, voire exclusive, sur les fonds anciens conduit à sous-estimer l'intérêt patrimonial à venir des autres collections, notamment contemporaines, alors qu'il est indispensable d'admettre que leur transmission, conjointement aux traces que nous ont laissées les époques antérieures, présente le même degré de nécessité. C'est bien ce qu'établissent par d'autres éléments de définition des collections patrimoniales, mais dans

6. Bertrand Calenge, Les politiques d'acquisition, Paris, Éditions du Cercle de la Librairie, 1994, p. 184. Sa notion même de collection " semi-patrimoniale ", qui rencontre imparfaitement la faveur des professionnels, est fondée sur un argument chronologique : " collection issue des fonds courants de la bibliothèque, ayant perdu sa fraîcheur immédiate... mais dont la bibliothèque ne peut ou ne veut se débarrasser » (ibid., p. 164). 
une formulation qu'on peut juger restrictive, ces mêmes commentaires de la Charte des bibliothèques.

\section{LE PATRIMOINE EST D'ABORD AFFAIRE DE TRANSMISSION}

La notion de patrimoine inclut nécessairement celle de transmission ; elle n'a de sens qu'au sein d'une communauté qui sert de cadre à cette transmission, qu'il s'agisse d'une famille, d'un groupement social, religieux ou géographique, ici naturellement les publics à desservir par l'établissement. Ce qu'une bibliothèque peut avoir la mission de transmettre, c'est évidemment le capital documentaire qu'elle a reçu des générations précédentes ; ce sont aussi les compléments qui viennent l'enrichir au fil du temps, quel que soit leur support, dès lors qu'ils attestent la permanence du service public et la continuité de son action.

Car le fonctionnement d'une bibliothèque se conçoit logiquement sur la durée : sans revenir sur les quelques fondations d'Ancien Régime encore actives de nos jours (voir plus haut encadré 1, p. 14), la plupart des bibliothèques patrimoniales peuvent se prévaloir de quelque 200 ans d'existence, et d'avoir vu passer de nombreuses pratiques documentaires, suivant les modes politiques, les types de publics, les consensus plus ou moins développés qui régissent pour un temps leur vie quotidienne. Ce sont les accidents d'une substance dont il faut dégager les caractéristiques pour affirmer son identité propre : même si les documents conservés dans une bibliothèque sont par essence multiples, on ne peut nier que chaque fonds possède son histoire et ses particularités, s'est accru par des effets conjoncturels, s'est donné sa propre physionomie qui distingue la bibliothèque de ses voisines, et qu'elle doit naturellement développer et transmettre.

Une première définition, presque tautologique, pourrait donc être celleci : le patrimoine d'une bibliothèque est l'ensemble des fonds qu'elle conserve, ou qu'elle acquiert, à des fins de transmission. Ce propos ne présume nullement qu'il se compose, pour tout ou partie, d'ouvrages anciens, rares ou précieux, comme on l'a vu pour le domaine public ; mais il associe la détermination du patrimoine à la communauté que dessert la bibliothèque, autant dire à des partenaires de statuts variés, susceptibles 
d'intervenir de manière active dans les choix documentaires de l'établissement.

\section{LE PATRIMOINE DES BIBLIOTHĖQUES DOIT FAIRE L'OBJET D'UNE DÉCISION}

Parmi les bibliothèques détentrices de collections considérées comme " patrimoniales », beaucoup subissent dans les faits la présence parfois massive de fonds qu'elles connaissent mal et dont la conservation, bien qu'absolument prescrite par un sommaire consensus, n'est pas assurée dans de bonnes conditions. Malgré le développement d'actions de coopération depuis le milieu des années 1980, le statut général de ce patrimoine automatique n'a guère avancé depuis l'époque du rapport Desgraves : ce qui doit progresser aujourd'hui pour faire évoluer les pratiques, c'est moins la mise en œuvre d'opérations matérielles de conservation que la réflexion politique sur la fonction patrimoniale des bibliothèques, et sa traduction dans les choix de pilotage de chaque établissement.

Autrement dit, l'existence et la gestion d'un patrimoine documentaire n'ont pas à relever d'un simple effet de circonstance qui placerait les bibliothécaires dans l'obligation d'assurer la transmission mécanique de fonds reçus d'une tradition complexe et parfois contradictoire : elle réclame de ces professionnels une véritable décision qui inscrive le patrimoine dans la conduite générale de la bibliothèque, et puisse durablement constituer la base de son identité. Cet engagement politique des bibliothécaires s'apparente à la responsabilité juridique évoquée plus haut ${ }^{7}$ par Gérard Cohen à propos de la définition du domaine public. Elle s'appuie sans doute sur certains préalables communs, mais elle doit en être différenciée puisqu'elle vise à d'autres fins. Nous pourrions cependant considérer, si nous avions à constituer aujourd'hui nos collections ex nihilo, que la détermination de ce patrimoine recouvre exactement le périmètre du domaine public.

Le souci du patrimoine devrait être en effet l'argument principal de la politique documentaire : on a bien compris que notre définition des

7. Voir l'article « Le droit du patrimoine écrit : d'où il vient ? Où il va ? », pp. 14-25. 
collections patrimoniales se fonde sur le contenu des documents, et sur son adéquation aux objectifs de l'établissement sur la durée. Cette approche doit conduire les professionnels à définir dans les collections des segments thématiques sans distinction d'âge ou de support, mais traités avec une exigence documentaire d'autant plus forte qu'elle s'appuiera sur des priorités locales : spécificités géophysiques, traditions économiques, personnalités représentatives, etc. Ces rubriques, plus ou moins étendues suivant la taille et les moyens de la bibliothèque, sont à considérer dans toute la profondeur historique de leur champ, de manière à constituer de véritables collections de référence qui spécialisent la politique patrimoniale ${ }^{8}$, sans préjudice de l'offre encyclopédique de premier niveau que doivent continuer d'assurer les bibliothèques de lecture publique.

Un travail de cette nature aura logiquement deux conséquences : l'examen critique des collections anciennes dont une partie seulement pourra s'inscrire dans ces priorités documentaires, et sera donc l'objet préférentiel des mesures de sauvegarde (conditionnement*, restauration), nonobstant d'éventuels désherbages dans les autres domaines ; et d'autre part, un niveau spécifique d'acquisitions, courantes ou rétrospectives, qui distingue à tout moment ces disciplines dans l'offre générale de la bibliothèque, et prépare les voies d'une transmission consistante et clairement assumée. On en finit de la sorte avec la " patrimonialisation » des collections courantes : par la manière dont la bibliothèque a délimité son patrimoine, et parce qu'elle en fait un argument de base de sa politique documentaire, les documents qu'elle détient à ce titre sont patrimoniaux depuis leur entrée dans les collections. Il faut préciser que leur conférer d'emblée ce statut n'empêche pas leur circulation, tout au moins pour un temps, ni leur prêt à domicile : c'est même l'occasion d'imaginer une communication

8. Pour une bonne part, les outils professionnels susceptibles d'évaluer la pertinence de collections thématiques dans toute la profondeur historique de leur champ doivent encore être inventés. On peut cependant, pour approfondir cette réflexion, critiquer la manière dont sont gérés aujourd'hui certains fonds locaux ou régionaux, visant plus ou moins à l'exhaustivité, et qui s'apparentent presque davantage à des entreprises de bibliophilie qu'à de la politique documentaire au sens strict. Bien au contraire, il s'agirait ici d'offrir, pour chaque discipline concernée, l'équivalent d'une bibliographie pluraliste et soutenue des productions contemporaines, et dans sa continuité, un échantillon riche et représentatif des grandes étapes historiques de son développement. C'est pourquoi cette politique, sauf dans le cas de collections anciennes d'une rare complétude, suppose également des acquisitions rétrospectives. 
particulière à leur sujet ${ }^{9}$, mettant en valeur auprès d'un public élargi les options patrimoniales de la bibliothèque et le sensibilisant aux exigences d'une entreprise de transmission dont il pourrait, sous diverses formes, être aussi l'un des acteurs.

\section{LA POLITIQUE PATRIMONIALE REND NÉCESSAIRE LA COOPÉRATION}

Le patrimoine ainsi déterminé se veut sélectif : il implique des choix documentaires qui spécialisent partiellement la bibliothèque dans sa politique d'acquisition, et totalement dans ses actions de conservation. Ce n'est pas une surprise : tous les professionnels sont aujourd'hui rompus à l'idée qu'on ne peut pas tout conserver, et qu'il n'est pas viable économiquement que chaque établissement sur son propre territoire joue le rôle d'une petite bibliothèque nationale, gardienne de tous les savoirs du monde. Mais la définition d'une telle politique est indissociable de l'ouverture de l'établissement vers l'extérieur, à la fois comme préalable et comme conséquence, auprès de partenaires dont l'intervention cadre et complète ses options préférentielles.

Comme le faisait observer Hélène Richard dans son rapport sur les formations patrimoniales présenté au ministre de la Culture en $2010^{10}$, la bibliothèque ne peut en effet déterminer de son propre mouvement, sans qu'ils soient admis et validés par des instances de concertation et de régulation, les axes prioritaires de sa politique patrimoniale : cette précaution garantit un double niveau de pertinence des choix opérés, au regard de la communauté que dessert directement la bibliothèque (rôle de la tutelle), mais aussi pour la préservation globale d'un patrimoine d'intérêt national

9. Les ouvrages des collections patrimoniales qui sont à même de circuler doivent être équipés de telle sorte qu'on puisse à peu près garantir leur conservation : reliure spécifique des livres ou périodiques, signalement particulier pour les documents audiovisuels, etc. Ces précautions pourront être complétées par un dispositif de contrôle régulier, pour procéder en cas de problème à des remplacements sans se voir opposer l'indisponibilité commerciale de l'ouvrage.

10. Hélène Richard, La formation aux questions patrimoniales dans les bibliothèques : rapport à M. le ministre de la Culture et de la Communication, Inspection générale des bibliothèques, septembre 2010 ( $\left.n^{\circ} 2010-016\right)$, consultable en ligne (mars 2012), p. 15, note 12. 
réparti sur l'ensemble du territoire, et dont il faut maintenir l'équilibre et la représentativité (rôle de la « communauté scientifique »).

C'est pourquoi la définition des collections patrimoniales doit être améliorée pour aboutir à la formule suivante, qui exprime la réflexion dans sa totalité : le patrimoine d'une bibliothèque est constitué de tous les documents, sans distinction d'âge, de nature ou de support, que ses missions l'amènent à conserver sans limitation de durée ${ }^{11}$.

Cet équilibre sera d'autre part atteint par le renforcement des entreprises de conservation partagée : les établissements d'un même territoire, par exemple d'une région, pourront conclure des partenariats documentaires sur la base de cette conception du patrimoine, afin de mettre en place un réseau dont chaque élément se définit par ses compétences disciplinaires. On cherchera bien sûr, sans en faire une condition nécessaire de l'exercice, à représenter le spectre encyclopédique dans sa totalité : s'il est fort peu probable qu'on y parvienne absolument, cet objectif pourrait au moins conduire à limiter certaines redondances, à définir plus rigoureusement, par des articulations concertées entre bibliothèques, la portée réelle de chaque choix disciplinaire.

Aux termes de cet accord, chaque bibliothèque participante s'engage à constituer des fonds de référence dans les domaines arbitrés en commun. Elle y fait porter une veille documentaire accrue, des efforts significatifs en matière d'acquisition et de conservation, et reçoit régulièrement des autres établissements du réseau, dans les conditions que prévoient les règlements en vigueur, des collections complémentaires qu'ils n'ont plus à conserver, puisque leurs options patrimoniales sont différentes. Réciproquement, elle transfère aux autres établissements, suivant le rôle qu'ils jouent dans le réseau, les collections dont elle n'entend plus assurer la conservation puisqu'elles ne représentent pas ses choix documentaires. Vis-à-vis de son public, elle est un point d'accès qui rend disponible une offre documentaire considérablement renforcée, puisqu'elle fédère les travaux parallèles d'autant de bibliothèques en voie d'enrichissement.

11. Bernard Huchet, "Le patrimoine ", in Yves Alix (dir.), Le métier de bibliothécaire, Paris, ABFÉditions du Cercle de la Librairie, 2010, p. 180. 
Le schéma qui s'esquisse de la sorte a pour intérêt de mettre en place entre les établissements concernés un partage intégral de leurs missions : l'objectif n'est plus de considérer la conservation comme une fin en soi, ou pire encore, de limiter les effets d'un accord à de simples mesures d'élimination concertée, comme il en est souvent des plans de conservation limités aux périodiques. Il revient à chaque bibliothèque d'affirmer sa personnalité par le développement conjoint de ses collections anciennes et modernes, mais aussi par l'approfondissement de ses compétences, et dans le respect d'une continuité qui correspond à l'évolution générale des connaissances : la politique du patrimoine cesse d'être l'attribut marginal de quelques spécialistes, confits dans leur aptitude à reconnaître les cuirs de reliure et les filigranes du Grand Siècle, pour intéresser vraiment « la bibliothèque dans son entier ».

Bien entendu, le recours massif aux nouvelles technologies conditionnera dès l'origine le fonctionnement de ce réseau : la constitution d'un portail bibliographique susceptible de moissonner les catalogues des bibliothèques, l'installation de moyens logistiques pour faire circuler à la demande les exemplaires d'un établissement à l'autre, qu'il s'agisse d'un exemplaire physique ou de numérisation à la volée, la mise en place d'outils de communication qui sachent mettre en valeur ces nouvelles formes de coopération figurent en bonne place dans les préalables du projet.

Les obstacles sont toutefois nombreux, qu'il ne faut pas sous-estimer : d'un point de vue réglementaire, les échanges de collections entre bibliothèques territoriales sont possibles, mais ils doivent être strictement cadrés ${ }^{12}$; le transfert des collections d'État peut également se voir prescrire par l'autorité préfectorale, que rien n'empêche de saisir dans le cadre d'un mouvement coopératif, mais selon des procédures parfois lentes et longues. Les règlements respectifs des établissements participants devront être harmonisés pour que l'accès du public régional aux ressources réparties soit assez équitable, etc. On fera valoir encore les difficultés rencontrées à vouloir concilier certaines tutelles, et le temps nécessaire à les

12. Quelques expériences de ce type sont en cours, par exemple dans la région Haute-Normandie les transferts de certains livres pour enfants dans le cadre du plan de conservation partagée des fonds pour la jeunesse, avec l'intermédiaire de l'agence régionale de coopération (ARL). 
convertir - bien des montagnes que la seule foi des professionnels ne suffira peut-être pas à déplacer.

Toutefois, l'enjeu professionnel est de taille : il revient à faire évoluer la conception traditionnelle du patrimoine pour en faire l'instrument d'une politique documentaire homogène. Même en l'absence du système coopératif qui lui donnera toute sa dimension, il s'agit d'introduire dans la bibliothèque la conviction que le patrimoine doit être choisi, et non subi et que ce choix s'opère dans la concertation ; qu'il articule au juste sentiment de l'histoire de l'établissement le souci légitime du service public, et qu'il est une garantie pour l'avenir des collections. 
3

\begin{tabular}{l|l} 
À QUOI SERT UNE RÉSERVE ET & par Laure Jestaz
\end{tabular} QU'Y METTRE ?

Avant de se demander à quoi sert une réserve, posons-nous la question de savoir ce qu'elle est, ce que sa présence signifie dans une bibliothèque, ce qu'elle représente ${ }^{1}$.

La réserve désigne le plus souvent un lieu particulier dédié à la conservation, espace circonscrit physiquement par des murs, un coffre, etc. Il peut également s'agir d'une réserve symbolique, seulement caractérisée au catalogue par des mentions particulières de cotation et de consultation. Néanmoins, de quelque ordre qu'elle soit, elle rassemble généralement des documents qualifiés de rares et de précieux, ces deux notions étant tout à la fois les clés de voûte assurant la cohérence et la pertinence de cet ensemble et les pierres d'achoppement auxquelles se heurtent les bibliothécaires lorsqu'il s'agit de les définir.

\section{LA RÉSERVE EST UN LIEU}

La réserve est le plus souvent une pièce particulière dans une bibliothèque, dont l'accès est restreint, fermé à clé, soumis à autorisation. Sanctuarisé en raison de ce qu'il contient, ce lieu n'a d'autre objectif que d'assurer aux documents qu'il recèle la plus grande protection contre toute dégradation, d'ordre humain, accidentel, ou constitutif de l'état du document (papiers de mauvaise qualité, dont la fabrication engendre une fragilité supplémentaire, feuillets* isolés ou d'un format inattendu, etc.). Il présente généralement (ou devrait présenter) des moyens de protection renforcés contre le vol, l'incendie ou l'eau, et être doté d'une surveillance hygrométrique particulière.

1. La définition la plus récente est due à Philippe Nieto : une réserve est $1^{\circ}$ « un ensemble de livres ou de documents [...] classés à part du fait de caractéristiques imposant des précautions de conservation particulières [...] et qui ne sont communiqués que sous certaines conditions "; $2^{\circ}$ " un local où est conservé un tel ensemble ». Cf. l'article " Réserve » dans le Dictionnaire encyclopédique du livre, Paris, Electre - Éditions du Cercle de la Librairie, 2011, III, pp. 552-554. 
Une réserve est donc un lieu dont on protège particulièrement le contenu :

- en sécurisant ces locaux contre le vol : mise sous clef des documents, qu'il s'agisse de pièces ou d'étagères grillagées, de coffres-forts ; obligation d'inscrire sur un registre chaque passage, qu'il s'agisse d'allers et venues liés à la communication des documents, de visites de personnalités, de groupes ; conservation des bulletins de communication des documents présents dans ces lieux ; interdiction d'y laisser seule une personne non habilitée ;

- en assurant dans ces locaux une veille climatique par le biais de capteurs hygrométriques régulièrement contrôlés. Un système de climatisation peut y être installé, avec toute la prudence requise puisque c'est le plus souvent par le biais de ces arrivées d'air en circuit fermé que se propagent les infestations lorsqu'elles surviennent ${ }^{2}$;

- en veillant à protéger ces lieux d'une trop forte luminosité susceptible d'abîmer les reliures et de jaunir les papiers : mise en place de volets, de filtres solaires, etc. ;

- en assurant un conditionnement propre à chaque type de document : boîtes de conservation sur mesure, acquisition de meubles à plans pour les documents de très grand format ou en feuillets..., et en entreprenant la restauration des pièces endommagées ;

- en s'assurant que le ménage y est fait par une personne sensibilisée à la conservation des documents, qui ne lavera pas le sol à grande eau, ne vaporisera pas sur les rayonnages des produits caustiques dommageables à la conservation, ne frottera pas les reliures poussiéreuses avec un chiffon empreint de produit chimique attaquant les matières organiques, etc. ;

2. Toute climatisation impose également une réflexion sur le choc thermique supporté par le document lorsqu'il doit sortir du lieu réfrigéré pour être consulté dans une salle de lecture à température ambiante. 
- en disposant sur place les instruments propres à la manipulation des documents : futons*, lutrins, sous-main, gants, crayons à papier...

On aurait tort de croire à cette lecture que la protection d'une réserve requiert de lourds moyens financiers. Certes, la mise en place de capteurs climatiques et l'installation de meubles de rangement adaptés sont des éléments d'un coût non négligeable. Mais la majeure partie des actions à mettre en place (ne pas y laisser seul un visiteur, ne pas travailler avec sa bouteille d'eau sur la table, ne pas entreposer des plaques de verre sur un coin de rayonnage dans l'attente de la boîte de conservation commandée...), requiert avant tout du bon sens, non de l'argent.

\section{LA RÉSERVE, ESPACE MENTAL DE CONSERVATION}

\section{DÉTERMINER UNE SEMI-RÉSERVE EN MAGASIN}

Mettre en place dans son institution un espace circonscrit physiquement et doté de protections maximales relève parfois de l'impossible. Le manque de place, de moyens financiers, de forces vives, peut l'interdire et reporter ce projet sine die. On peut alors déterminer une sorte de semi-réserve en magasin, qui dotera les documents concernés d'un statut particulier et suscitera pour eux des conditions sans doute minimales mais particulières de communication, voire de conservation, tout en les maintenant au milieu des autres documents de la bibliothèque.

Il sera bon de les distinguer alors au catalogue par une cote singulière qui alertera les lecteurs et les bibliothécaires sur leur fragilité, leur rareté, leur valeur, et induira des modes de rangement et de communication spécifiques, au sein des magasins et dans la salle de lecture :

- mise en boîte de conservation ou restauration si les budgets le permettent ;

- espace de consultation particulier en salle de lecture avec une surveillance accrue, utilisation de futons et de lutrins, interdiction du stylo à encre... ;

- interdiction d'emprunt par des particuliers ; 
- conditions spécifiques de reproduction (sans flash, sur un support adapté pour éviter de casser la reliure...).

\section{LA RÉSERVE, UN ENSEMBLE DE DOCUMENTS PARTICULIERS}

C'est par les documents qui y sont conservés que la réserve, espace de protection, acquiert son statut de sanctuaire ou de "saint des saints » (sanctum sanctorum, pour reprendre la terminologie de nos collègues anglais) se dotant parfois d'une majuscule et de qualificatif (notons que l'expression "Réserve précieuse " est encore une dénomination chère à nos institutions occidentales ${ }^{3}$ - signifiant par là qu'il existe bel et bien des réserves non précieuses).

Parfois bibliothèque à part entière au sein de l'établissement, dotée de règles propres, de son propre personnel, la réserve reste la partie la plus prestigieuse des bibliothèques ${ }^{4}$. Ce sont ses documents qui sont présentés aux tutelles et aux visiteurs de marque, ce sont eux qui assurent son rayonnement (par le biais d'expositions, d'études scientifiques...), c'est leur sauvegarde qui doit être privilégiée en cas de sinistre. Sur quels critères est donc constitué un ensemble d'une telle valeur?

Le plus souvent, on hérite d'une réserve déjà constituée. On pense à l'enrichir, on pense moins à l'évaluer au regard de l'intérêt des documents et de la politique documentaire de la bibliothèque ; on pense enfin très rarement à la désherber (on entend par là refouler dans le fonds général). La réserve est cependant le lieu de tous les amoncellements, là où ont été mis tout ensemble les unica (manuscrits, dessins...), les monuments typographiques universellement renommés (les incunables*, les grands livres $\mathrm{du} \mathrm{XVI}^{\mathrm{e}}$ siècle...), les photographies sans doute anciennes mais encore si souvent terra incognita des bibliothécaires qu'on ne sait trop les analyser, les objets improbables (buste d'une célébrité locale, médailles, boîtes de marqueterie, toiles encadrées...). La confusion entre réserve et cabinet de curiosités est encore fréquente, héritage vivace d'un passé qui n'a su que faire d'objets hétéroclites de provenances multiples.

3. C'est notamment le cas en Belgique à la Bibliothèque Royale, à l'Université libre de Bruxelles et à Mariemont, en Suisse à Lausanne...

4. Au contraire des musées où la réserve est un lieu où l'on stocke, souvent en grand nombre, les œuvres que l'on n'expose pas et qui sont généralement de moindre valeur. 


\section{CE QU'ON TROUVE DANS UNE RÉSERVE}

La volonté de mettre en réserve, c'est-à-dire à part, par précaution, les documents d'une valeur et d'un intérêt méconnus est aujourd'hui singulièrement freinée par le manque de place dans les bibliothèques, ou mise à mal par le réaménagement des lieux (notamment dans le cadre d'un déménagement). Les espaces de réserve, lieux de stockage de varia plus ou moins précieux, explosent. Les normes de conservation, toujours plus draconiennes et directives, impliquent souvent le desserrement des documents sur une étagère, de nouveaux modes de rangement requérant davantage de place... Quels documents doivent donc y être conservés, et quelle doit être au $\mathrm{xxI}^{\mathrm{e}}$ siècle la politique d'enrichissement d'un tel ensemble?

Quelques grandes lignes de force sur la constitution des réserves de bibliothèques peuvent être soulignées. L'encadré ci-dessous présente les documents le plus souvent conservés.

\section{ENCADRÉ LES DOCUMENTS À METTRE EN RÉSERVE}

- les manuscrits, toutes époques confondues, et plus généralement les unica : dessins, recueils d'aquarelles, relevés d'architecture, épreuves corrigées... Pièces impossibles à racheter en cas de perte, souvent fragiles, témoins irremplaçables d'une vie quotidienne, d'un travail en cours, dont l'unicité n'est pas toujours pour autant gage de grande valeur - mais la sélection, difficile, des manuscrits à préserver en priorité est rarement établie ;

- les documents témoignant de la naissance et de l'histoire de leur art : les incunables (du livre, des estampes, de la photographie...), les grands monuments typographiques et iconographiques ou audiovisuels - dont il serait pourtant judicieux de vérifier l'absence d'autres exemplaires au sein des ressources locales avant leur mise en réserve ;

- les livres interdits, à caractère raciste, érotique ou pornographique. Leur mise en réserve est en ce sens moins liée à un souci de conservation qu'à une vigilance particulière à apporter à leur consultation. Il peut s'agir au reste de documents assez rares car mal diffusés, poursuivis, etc. ;

- les documents précieux par rapport aux fonds de la bibliothèque, eu égard à :

- des particularités d'exemplaire (provenance illustre d'intérêt national ou local, exemplaire truffé, 
annoté, avec envoi, épreuves corrigées...), leur valeur étant liée à l'histoire du livre ou de la bibliothèque d'origine ;

- l'ancienneté des documents : de manière quasi générale, les imprimés des $\mathrm{XV}^{\mathrm{e}}-\mathrm{XvI} \mathrm{e}^{\mathrm{e}}$ siècles se retrouvent en réserve. Étendre ce critère aux siècles suivants dépend de la taille de l'institution et de la volumétrie des fonds concernés ;

- la rareté des documents. Très difficile à évaluer avant l'ère des catalogues informatisés, cette notion est aujourd'hui bien plus facile à appréhender mais doit être maniée avec prudence. On doit rester prudent par rapport aux informations accessibles : ces catalogues n'étant pas encore tous exhaustifs ni mis à jour, l'unicité d'un exemplaire ne peut être réellement assurée ;
- les reliures d'une qualité remarquable, eu égard aux matériaux employés, à la technique de fabrication, à la provenance locale du relieur... ;

- les livres particulièrement fragiles et qui requièrent une conservation particulière ou dont on sait qu'ils n'ont pas leur place au fonds général en raison de leur format (des minuscules qui se perdraient parmi d'autres imprimés, des panoramas enroulés, des recueils de gravures in-plano...). Une attention particulière doit être portée aux périodiques, que la mauvaise qualité $\mathrm{du}$ papier rend très fragiles et dont on croit, bien souvent à tort, qu'ils sont de diffusion courante et donc conservés ailleurs ;

- Les livres de bibliophilie à tirage restreint ou les livres d'artiste, exemplaires souvent uniques par l'insertion d'œuvres iconographiques originales, justifiés, etc.

\section{QUELLES QUESTIONS SE POSER ?}

La réflexion préalable doit porter sur une réserve idéale, qui ne serait pas contrainte par des problèmes d'espace, de budget, de personnel. Il s'agit dans un premier temps de définir, par type de document, les critères conduisant à une mise en réserve avant de confronter, dans un second temps, ses désirs à la réalité pratique et matérielle du moment. La réflexion sur la constitution d'une réserve et sur son accroissement doit également mener à s'interroger sur la place qu'occupe cet ensemble par rapport au fonds général de la bibliothèque, ou par rapport au fonds ancien s'il existe. 
- En quoi la réserve est-elle différente du fonds ancien ? Estelle assimilable au fonds patrimonial ? au fonds local ? au fonds dit de conservation ? Quelle est sa spécificité par rapport à ces derniers, quelle est son identité propre ?

- Quel type de document doit-on mettre dans cet espace ? Si la bibliothèque recèle des documents très différents (plaques de verre, microsillons, imprimés, films en nitrate, recueils de gravures...) nécessitant chacun des conditions de conservation différentes, doit-on créer plusieurs réserves ? Quels liens créer entre elles en ce cas, en termes d'espace, de communication, d'emprunt... ?

Il peut être intéressant de distinguer une réserve ancienne, historique, souvent encyclopédique en termes de contenu, dont l'un des critères principaux est la date de publication du document ; et une réserve dite moderne, constituée selon des principes plus restrictifs liés à l'histoire locale, à la vulnérabilité du document, à un tirage... La seconde n'est pas nécessairement le prolongement de la première même s'il importe d'établir entre elles des relations de connivence en fonction de l'intérêt des fonds et des caractéristiques matérielles des documents.

\section{LES GRANDS PRINCIPES D'UNE RÉSERVE}

L'intention n'est pas ici de porter un regard négatif sur ce qui a été fait précédemment. Les remarques qui suivent sont l'objet d'un constat. Et ce sont les contraintes du moment (contraintes d'espace, contraintes budgétaires...) qui obligent, sainement, à porter un regard critique sur les actions précédentes avec l'espoir d'en dégager certains grands principes.

\section{Établir des critères par type de document}

Bien qu'elle ait été le plus souvent constituée selon des critères implicites, souvent liés à la personnalité des prédécesseurs en poste et à leurs 
intérêts, la réserve doit aujourd'hui être l'œuvre d'un choix (critères de date, de fragilité, de la présence d'iconographie...) ${ }^{5}$.

Ce choix doit être étroitement dépendant des fonds de la bibliothèque, de sa spécificité locale, de son histoire, et de la politique documentaire établie.

Ce choix doit considérer tout à la fois et séparément la rareté, la fragilité et l'unicité d'un exemplaire, qui permettent d'en définir la valeur. C'est en croisant l'ensemble de ces critères qu'il importe de décider d'une mise en réserve.

Ce choix doit être raisonné par type de document : un tirage tardif d'une célèbre gravure de Daumier, un tirage original de Charles Marville, un dessin très abouti de Victor Hugo n'auront ni la même valeur, ni la même rareté et ne requerront pas les mêmes conditions de conservation, de protection, de communication.

\section{Faire des choix en concertation}

Ce choix doit être fait collégialement. Quelle que soit la taille de la bibliothèque, ce serait une erreur que de réfléchir par département ou par service : le responsable des acquisitions courantes, de la lecture publique, le responsable de la section jeunesse, celui des cartes et plans, du multimédia, des manuscrits, doivent être sollicités à part égale dans cette réflexion qui doit associer documents anciens et modernes, ceux dont le caractère précieux est avéré et ceux pour lesquels il faut réfléchir dès leur acquisition sur la conservation qu'on souhaite leur offrir. La patrimonialisation d'un document est l'œuvre de chaque jour, elle est une réflexion permanente et vivante, abstraction faite de l'ancienneté de l'exemplaire acquis.

\section{Rédiger une charte}

Ces grands principes, qui fondent l'identité d'une réserve, doivent faire l'objet d'une charte écrite et connue. Ce document de référence sera une aide objective pour les acquisitions patrimoniales futures (on aurait tort

5. Voir Antoine Coron, « La réserve des livres rares à la Bibliothèque nationale de France : constitution d'une nouvelle collection ", Bulletin des bibliothèques de France, 1996, t. 41, n³, pp. 20 25. [En ligne] < http://bbf.enssib.fr/consulter/bbf-1996-03-0020-004 >. 
de négliger la part d'affectivité inhérente à ce type d'achat, si humaine qu'elle reste aujourd'hui encore un critère rarement affiché mais bien vivant), qui aidera les bibliothécaires suivants dans la poursuite de cette politique.

Toutefois, parce qu'une bibliothèque est une institution vivante dont les collections fluctuent au gré des budgets, des réaménagements, des personnes en poste et des lecteurs qui l'utilisent, il n'est pas rare qu'elle reçoive des dons d'importance susceptibles de bousculer, plus ou moins fortement, son identité, et par ricochet celle de la réserve déjà constituée. Des aménagements devront être faits, qui inscriront dans le temps le nouvel axe d'enrichissement retenu en lui conférant un poids et une signification dans son développement futur. La réserve d'une bibliothèque doit demeurer un lieu vivant, non un musée de collections précieuses.

\section{DES DOCUMENTS SOUMIS À DES RÈGLES DE COMMUNICATIONS SPÉCIFIQUES}

La présence d'une réserve induit généralement la mise en place de règles de consultation spécifiques pour ses documents : afin de garantir leur sécurité, ceux-ci seront consultés dans un espace particulier de la salle de lecture, sous la surveillance d'un bibliothécaire, avec mise à disposition d'instruments facilitant leur manipulation : futons, lutrins, boudins... L'autorisation d'un responsable peut être requise, notamment dans le cas de pièces particulièrement précieuses, et ce qui pourrait ici apparaître comme une contrainte peu pratique au sein de l'établissement lui est souvent profitable en ce qu'elle offre une meilleure connaissance de son public et favorise l'insertion de la bibliothèque dans le monde de la recherche en contribuant à son rayonnement. 


\section{4 \\ COMMENT (BIEN) CONSERVER LES COLLECTIONS PATRIMONIALES? \\ par Jocelyne \\ Deschaux}

Contrairement à une idée longtemps répandue, le patrimoine écrit ne se conserve pas " tout seul ", posé sur un rayonnage. La conservation préventive est essentielle pour la bonne préservation des collections et pour atteindre au moins deux de nos objectifs professionnels : l'accessibilité de tous les publics d'aujourd'hui à ces collections, et la transmission de celles-ci aux générations futures.

\section{LE PATRIMOINE ÉCRIT : LES PRINCIPAUX MATÉRIAUX ET LEUR CONSTITUTION}

Les matériaux qui constituent principalement les collections patrimoniales en bibliothèque (hors photographies) sont essentiellement du parchemin (peau de mouton, de veau ou de chèvre), du papier chiffon (lin, chanvre ou coton, aux longues fibres de cellulose, très résistantes) encollé à la gélatine, du papier bois (comportant de la lignine) encollé à la résine, du cuir, des colles animales, des nerfs (d'origine animale), des ficelles : ce sont donc des matériaux organiques d'origine végétale ou animale. Cela a trois conséquences :

- ce sont des matériaux hygroscopiques, c'est-à-dire qui réagissent à l'humidité de l'air : en l'absorbant, ou en rejetant de leur propre humidité (cf. infra p. 48);

- ce sont des matériaux qui peuvent servir de nourriture à toute une faune (les insectes), à une flore (les moisissures) et aux bactéries ;

- ce sont des matériaux (surtout le cuir) qui continuent à « vivre », qu'il faut entretenir et nourrir.

Il est essentiel de veiller aux différents aspects de la conservation préventive dans tous les moments de la vie en bibliothèque, que cela soit au 
niveau du stockage, ou à celui de la consultation, ou encore de la valorisation de ces collections.

\section{CONSERVATION DANS LES MAGASINS}

Les principaux agents de dégradation sont : les conditions climatiques, la lumière, la pollution, les agents biologiques (insectes, champignons), les hommes...

\section{LA TEMPÉRATURE ET L'HYGROMÉTRIE*}

\section{Définitions}

L'hygrométrie est la teneur en eau de l'air environnant.

Tout volume d'air contient une certaine quantité de vapeur d'eau à une température et une pression données : c'est l'humidité absolue (mesurée en $\mathrm{g} / \mathrm{m}^{3}$ ). La capacité de l'air à contenir de la vapeur d'eau dépend de la température : plus l'air est chaud, plus il peut contenir de vapeur d'eau. L'humidité relative* (HR) est le rapport de cette quantité de vapeur d'eau par la capacité d'absorption de l'air à la même température. Cette relation est exprimée en pourcentage.

Quantité de vapeur d'eau présente dans un $\mathrm{m}^{3}$ d'air à une température donnée

Humidité relative $=$ $\mathrm{x} 100$

Quantité maximale de vapeur d'eau pouvant être contenue dans ce $\mathrm{m}^{3}$ d'air à la même température

On appelle point de rosée*, à une température donnée, le point où l'humidité relative est égale à $100 \%$ : l'air est saturé et ne peut plus contenir davantage de vapeur d'eau. Au point de rosée, l'eau se condense sous forme liquide, par exemple sur les surfaces froides (vitres, rayonnages métalliques...).

L'humidité relative est en relation directe avec la température : si la température augmente, l'humidité relative diminue (puisque plus chaud, l'air 
peut contenir plus d'humidité, l'air est donc moins saturé, et l'HR diminue); et inversement.

À humidité absolue donnée, dans un local donné sans apport de vapeur d'eau extérieur, un degré en moins augmente l'humidité relative de $3 \%$.

\section{Les conséquences de la température et de l'humidité sur les documents}

Les matériaux organiques sont extrêmement hygroscopiques : ils réagissent donc directement aux conditions climatiques.

\begin{tabular}{|c|c|c|}
\hline situation climatique & conséquence & $\begin{array}{l}\text { Type de document } \\
\text { concerné }\end{array}$ \\
\hline $\mathrm{T}^{\circ}$ élevée & $\begin{array}{l}\text { Dégradation des matériaux } \\
\text { (surtout instables) }\end{array}$ & Papiers acides \\
\hline $\mathrm{T}^{\circ}$ et HR élevées & Développement des moisissures & Tous \\
\hline $\mathrm{T}^{\circ}$ élevée et HR basse & $\begin{array}{l}\text { Dessèchement des matériaux, } \\
\text { friabilisation }\end{array}$ & Cuirs et papiers \\
\hline $\mathrm{T}^{\circ}$ basse & $\begin{array}{l}\text { Friabilisation des matières } \\
\text { organiques }\end{array}$ & Papiers \\
\hline HR élevée & Corrosion des matériaux & $\begin{array}{l}\text { Fermoirs, } \\
\text { boulons* métalliques }\end{array}$ \\
\hline
\end{tabular}

Des fluctuations de la température et de l'humidité relative se traduisent par des phénomènes d'absorption et de désorption d'eau, ayant pour conséquence des variations dimensionnelles des matériaux qui entraînent à la longue une fatigue mécanique : il y a perte d'élasticité, de souplesse et de solidité : on constate des fendillements et des décollements. Attention, les documents ayant subi une restauration récente sont particulièrement sensibles aux variations d'humidité.

\section{comment éviter ces dégradations?}

\section{Respecter les conditions de conservation recommandées}

Pour nos climats tempérés, et pour les documents en papier et en cuir, les recommandations sont les suivantes : $18^{\circ} \mathrm{C} \pm 1^{\circ} \mathrm{C}$ et $55 \%$ d'HR $\pm 5 \%$. 
Des fluctuations sont généralement admises entre 16 et $21^{\circ} \mathrm{C}$ et $40 \%$ et $60 \%$ d'HR, à condition que ces variations ne soient pas brutales, mais saisonnières.

L'essentiel est surtout de stabiliser la température et le taux d'humidité pour limiter au maximum les variations climatiques.

Pour éviter le développement de moisissures, il faut impérativement ne pas dépasser $60 \%$. Une variation maximale de $5 \%$ sur 7 jours avec $1 \%$ par 24 heures est admise.

\section{Connaître les conditions thermo-hygrométriques dans les magasins}

Il est donc essentiel de connaître les conditions climatiques dans les magasins, afin de détecter une panne ou une anomalie, d'installer un matériel adapté - chauffage et/ou climatisation, dés/humidificateurs, matériaux tampons (gel de silice), etc. -, de pouvoir dialoguer avec d'autres corps de métier : maintenance, climatisation, architectes...

Les mesures de température et humidité relative se prennent avec des thermo-hygromètres* enregistreurs (thermo-hygrographes), à relevé plutôt hebdomadaire que mensuel, que l'on placera dans une zone climatique représentative et loin d'une bouche de soufflage d'air, ou de voies de passage. On n'oubliera pas de les étalonner régulièrement (la fréquence d'étalonnage dépend de la nature de l'appareil, mécanique ou électronique), et d'en analyser régulièrement et correctement les relevés.

\section{Les mesures à prendre}

Agir sur l'un des deux facteurs (la température ou l'humidité relative) a des conséquences sur l'autre.

\section{ACTIONS POSSIBLES POUR AMÉLIORER}

\section{TABLEAUX LES CONDITIONS CLIMATIQUES}

\begin{tabular}{|l|l|l|l|l|l|}
\hline Si & on peut & en & Si & mais & il faut \\
\hline $\begin{array}{l}\text { Air trop } \\
\text { sec }\end{array}$ & $\boldsymbol{\pi} \mathrm{HR}$ & $\mathbf{y} \mathrm{T}^{\circ}$ & Air trop sec & $\mathrm{T}^{\circ}$ bonne & humidifier l'air \\
\hline $\begin{array}{l}\text { Air trop } \\
\text { humide }\end{array}$ & $\boldsymbol{y} \mathrm{HR}$ & $\boldsymbol{\pi} \mathrm{T}^{\circ}$ & Air trop humide & $\mathrm{T}^{\circ}$ bonne & déshumidifier l'air \\
\hline & & & $\mathrm{T}^{\circ} \mathbf{y}$ & HR bonne & déshumidifier l'air \\
\hline $\mathrm{T}^{\circ} \boldsymbol{\lambda}$ & HR bonne & humidifier l'air \\
\hline
\end{tabular}




\section{LA LUMIÈRE}

\section{Qu'est-ce qui dégrade?}

La lumière, naturelle ou artificielle, est nocive aux documents : toutes les longueurs d'ondes de la lumière encouragent la décomposition chimique des matériaux organiques par oxydation. La lumière peut faire blanchir, jaunir ou brunir certains papiers ; elle décolore certains pigments, altérant la lisibilité et l'apparence des documents.

L'œil humain ne voit pas l'infrarouge (IR) ni l'ultraviolet (UV). Ce sont pourtant deux radiations qui sont nocives pour les documents.

\section{EFFETS ET CONSÉQUENCES DES RADIATIONS}

\section{TABLEAU SUR LES DOCUMENTS}

\begin{tabular}{|c|c|c|}
\hline Radiation & Effets & conséquences sur les documents \\
\hline UV & $\begin{array}{l}\text { Dégradations photochimiques } \\
\text { de la matière }\end{array}$ & $\begin{array}{l}\text { Jaunissement des papiers contenant } \\
\text { de la lignine }{ }^{1} \text {, } \\
\text { effacement des pigments, } \\
\text { perte de résistance mécanique }\end{array}$ \\
\hline IR & $\begin{array}{l}\text { Dégradation par échauffement, } \\
\text { dessèchement des matériaux }\end{array}$ & Accélération du vieillissement \\
\hline
\end{tabular}

La lumière artificielle est aussi nocive. Les lampes à incandescence (à ampoules, halogènes) dégagent des infrarouges : leur effet est donc chauffant. Les lampes à fluorescence (de type néon) dégagent beaucoup d'UV. Les lampes à fibre optique produisent une lumière froide (donc qui n'émet pas de chaleur).

Il faut donc éliminer l'IR et l'UV, et éviter l'éclairage direct sur les documents.

\section{comment protéger les documents de la lumière lors du stockage $\mathbf{~}^{\mathbf{2}}$}

- ne pas exposer ou stocker de documents directement aux rayons du soleil ;

- munir les fenêtres de stores, de rideaux, de volets et/ou de filtres / films ;

- éteindre systématiquement quand il n'y a personne dans le magasin.

1. Un des principaux composants du bois, responsable du jaunissement du papier fabriqué à partir de la fibre de bois, après exposition au soleil.

2. Voir plus loin la partie sur les expositions et la reproduction p. 59. 


\section{LA POLLUTION}

\section{Qu'est-ce qui dégrade ?}

Il faut savoir que les polluants sont également nocifs pour les documents.

\section{TABLEAU LES EFFETS DE LA POLLUTION}

\begin{tabular}{|c|c|c|}
\hline Polluants & Effets & conséquences \\
\hline $\begin{array}{l}\text { Solides } \\
\text { (aérosols, poussières, } \\
\text { fumées, brouillards) }\end{array}$ & $\begin{array}{l}\text { Dépôt de particules } \\
\text { infimes sur les } \\
\text { documents }\end{array}$ & $\begin{array}{l}\text { Graisse les supports } \\
\text { Dégradations mécaniques } \\
\text { par abrasion } \\
\text { Terrain propice au } \\
\text { développement des moisissures }\end{array}$ \\
\hline $\begin{array}{l}\text { Gazeux } \\
\text { - Ozone (dégagée par } \\
\text { photocopieurs et } \\
\text { purificateurs d'eau) } \\
\text { - Pollution industrielle } \\
\text { (dioxyde de soufre) }\end{array}$ & $\begin{array}{l}\text { Oxydation et } \\
\text { hydrolyse des } \\
\text { macromolécules } \\
\text { - Oxydation } \\
\text { - Scission des } \\
\text { matériaux }\end{array}$ & $\begin{array}{l}\text { Dégradations chimiques } \\
\text { - Documents papier et } \\
\text { photographies } \\
\text { - Fragilisation des documents }\end{array}$ \\
\hline
\end{tabular}

L'air non filtré est aussi la principale source de poussières et de spores de moisissures.

La pollution vient aussi de certains matériaux utilisés ${ }^{3}$ : certains bois, certains textiles utilisés dans des vitrines d'exposition, certaines peintures, colles et solvants ; les cartons et papiers ordinaires ; les plastiques à base de PVC (présence de chlore) ; les élastiques (présence de soufre) ; le scotch ; les nitrates de cellulose et acétates de cellulose (contenus dans certaines photographies) dégagent des gaz toxiques.

\section{comment éviter ces dégradations ?}

Lorsque les locaux sont climatisés, le niveau de pollution peut être réduit grâce à l'utilisation de filtres à charbon actif qui doivent être changés régulièrement. Les filtres électrostatiques sont à éviter car ils dégagent de l'ozone.

3. Voir l'annexe D de la norme AFNOR Z 40-010 relative aux conditions de conservation des documents graphiques et photographiques pendant leur exposition, qui donne la liste des matériaux nocifs aux documents. 
Un dépoussiérage* régulier des collections est indispensable, car très efficace pour minimiser ces risques de dégradations. Les aspirateurs doivent être munis de filtre absolu* pour éviter que des spores de micro-organismes se dispersent à nouveau dans l'air.

Et puis :

- ne pas placer de photocopieur près des collections, notamment photographiques ;

- éviter les rayonnages et meubles en bois, les cadres en bois pour les expositions (bois interdit pour les photos !) ;

- utiliser des peintures acryliques ;

- attendre au moins trois semaines avant de remettre des collections dans un local qui a subi des travaux de peinture, pose de revêtement de sol... et aérer régulièrement le local en attendant ;

- choisir des conditionnements en matériaux neutres*.

\section{LES AGENTS BIOLOGIQUES : INSECTES, CHAMPIGNONS, BACTÉRIES}

Les agents de dégradation biologique (insectes et moisissures) contribuent de façon importante à la dégradation des collections de bibliothèque. Ces agents se nourrissent des substrats organiques qu'ils trouvent dans les documents ou dans la poussière qui les recouvre. L'absence de ventilation, l'obscurité et des niveaux de température et d'humidité relative élevés favorisent leur développement. Les dommages causés (destruction des papiers et des reliures, taches...) sont irréversibles.

\section{Quelles dégradations?}

\section{Les insectes}

La présence d'insectes est détectable à divers signes : sur les documents eux-mêmes, on peut trouver des galeries sinueuses pour les lépismes* et les dermestes*; de petits trous circulaires pour les vrillettes* ; de petits monticules de sciure révélant l'existence d'insectes xylophages ou de petits tas d'excréments (poussières noires). Enfin, on constate parfois directement la présence d'insectes dans les magasins. 
En fait, dans nos climats tempérés, les attaques d'insectes sont rarement de grande ampleur.

\section{Les champignons / moisissures}

Les champignons papyricoles s'attaquent tout particulièrement aux collections patrimoniales. Plus de 600 espèces ont été recensées. Ces moisissures sécrètent des pigments qui diffusent dans le papier en laissant des taches de couleurs différentes, sur les dos, plats, tranches, premiers cahiers*, marges extérieures, ou toute partie du livre qui a été mouillée. $\mathrm{Au}$ toucher, on constate un affaiblissement de la structure du papier dû à la « digestion » de la cellulose, parfois même une apparition de trous.

\section{comment éviter le développement des moisissures ?}

Les spores de moisissures sont partout dans l'air environnant, apportées par l'air, l'eau, ou par contact. Certaines spores peuvent rester à l'état " latent » plusieurs années avant la germination. Leur développement est étroitement lié aux conditions climatiques, et en particulier à l'humidité relative (si elle est supérieure à $62 \%$, le risque est très important). Il est favorisé aussi par la présence d'éléments nutritifs : poussières, cuirs, colles, papier. Un facteur déclenchant est généralement nécessaire : apport anormal d'humidité par fuite, infiltration, rupture de canalisation... La germination entraîne le cycle de la moisissure. Celle-ci se développe en se nourrissant des composants des documents et produit à son tour des spores, même après diminution de l'humidité relative. La propagation des infestations est très rapide.

Les moisissures présentent un aspect pulvérulent : aspect de mousse ou de poudre, relief de la tache visible en lumière rasante. 


\section{Que faire en cas de développement ou infestation de moisissures ?}

Si cela arrive, il faut stopper le facteur déclencheur, déshumidifier le local, isoler, sécher et dépoussiérer les documents atteints (hors du magasin) avec un aspirateur à filtre absolu, et si le local est aussi infesté, dépoussiérer le magasin. Une surveillance devra ensuite être attentive pendant 6 mois.

Le personnel doit se protéger (gants, masque et blouse pour les manipulations) et limiter au maximum les déplacements dans le magasin.

Tous les conseils pourront être obtenus auprès de la $\mathrm{BnF}^{4}$ ou du Centre de recherche et de conservation des collections (CRCC) ${ }^{5}$.

Si toute une collection est concernée, on pourra avoir recours à la désinfection* des collections à l'oxyde d'éthylène, ou par anoxie* pour une infestation par insectes ; suivie de la désinfection des locaux. Mais la désinfection est un procédé très agressif pour les documents et elle n'a aucun caractère préventif. Elle est donc réservée aux cas les plus résistants et aux grandes quantités. Le dépoussiérage soigneux suffit dans la grande majorité des cas.

\section{LE StOCKage et LA MAINTENANCE des COLleCtionS}

Les modes de stockage ont une influence directe sur la vie des documents. De mauvaises conditions endommagent rapidement les collections, provoquant des déformations (espaces entre les étagères non adaptés à la hauteur véritable des ouvrages, absence de serre-livres pour des étagères non complètes...), ou des éraflures sur les reliures (rayonnages métalliques à arêtes vives, extraction violente des rayonnages). C'est aux rayonnages de s'adapter aux collections, et non l'inverse.

\section{Les rayonnages et mobilier}

- Le sens des travées doit être perpendiculaire à la position des bouches de soufflage de la ventilation ou de la climatisation, afin d'assurer une bonne circulation d'air à l'intérieur des rayonnages, et aux fenêtres afin d'éviter un impact direct de la lumière de jour.

4. Département de la Conservation : < desinfection@bnf.fr >.

5. CRCC : $<$ http://www.crcc.cnrs.fr $>$. 
- Il ne faut pas placer les rayonnages tout contre les murs extérieurs afin d'éviter les conséquences néfastes de possibles infiltrations ou condensations.

- Lorsque des documents sont rangés dans des armoires fermées, on doit s'assurer que celles-ci sont ventilées correctement. Les ouvertures d'aération doivent se trouver sur les côtés et non pas en haut pour éviter la chute de poussière.

Du point de vue de la conservation, il faut aussi être vigilant à l'absence d'aspérités pouvant provoquer des éraflures sur les reliures, à la facilité de montage et de démontage, à la surface antidérapante des tablettes, à leur résistance à la charge, à la qualité de la peinture, à la douceur de déplacement d'un compactus et à l'espace laissé toujours libre entre les épis pour la circulation de l'air.

Pour les documents en feuilles (estampes, cartes, dessins, plans...), souvent pliés (cartes) ou empilés (estampes), il faut utiliser soit des armoires à grands tiroirs plats, soit des boîtes de rangement disposées à plat sur deux largeurs de rayonnages.

\section{Rangement des documents}

Il est conseillé de ranger les documents par format, sans trop les serrer, debout jusqu'au format in-folio, couchés pour les plus grands ; en laissant derrière les livres un espace pour la circulation de l'air (ainsi qu'entre le fond des rayonnages et le mur).

Ne jamais, même temporairement, stocker des ouvrages au sol (même en cartons).

Il est aussi recommandé de surélever les premiers niveaux de rayonnages à 10-15 cm du sol environ afin de réduire les conséquences d'une inondation, en laissant sous la dernière tablette en bas l'espace accessible aux raclettes ou aux aspirateurs pour le ménage et l'assèchement du sol ; et d'utiliser des rayonnages équipés d'une tablette supérieure pour protéger les documents de la poussière et de la lumière. 


\section{Entretien des locaux}

La propreté des magasins (sols) et des rayonnages est tout aussi importante que celle des documents eux-mêmes : le rythme minimum de nettoyage est d'une fois par an, à l'aspirateur à filtre absolu, sans négliger l'arrière, le dessus et le dessous des rayonnages.

\section{La protection des documents}

\section{Le conditionnement}

La mise en boîte de conservation - en matériaux neutres - est l'une des meilleures actions de préservation, car elle permet de protéger les documents de la poussière, des aspérités des autres livres (fermoirs ou boulons), lors du transport, lors d'un sinistre (protection contre l'eau ou lors d'une évacuation), de préserver des décors de reliures, des documents en feuilles, des ouvrages restaurés ou au contraire fragilisés mais qu'on ne souhaite pas restaurer.

\section{Élimination des corps étrangers}

Doivent être ôtés tous les éléments qui peuvent abîmer les livres (par la rouille ou provoquant des déchirures) : agrafes, trombones, épingles... Si certains (fleurs séchées, images...) doivent être conservés, il est conseillé de les mettre dans la boîte du document, dans une pochette transparente neutre et étiquetée.

De même, on ne collera pas sur les documents patrimoniaux ni étiquettes de cote, de RFID, ni codes-barres, ni directement sur les feuillets ni sur les reliures ; les cotes pourront être notées sur des signets de papier permanent, où pourront également être collés (à la colle de pâte) les autres éléments cités.

\section{Maintenance des collections}

La deuxième meilleure action de préservation des collections est leur dépoussiérage, par un personnel formé et sensibilisé, muni de protections (blouse, gants, masque), et de chiffons doux non pelucheux, brosses souples, pinceaux, ou aspirateurs équipés de variateurs de puissance et de filtre absolu, en veillant à adapter le matériel en fonction de l'état et de la 
nature du document : si un document est fragile, une forte aspiration peut provoquer des déchirures. Ce matériel doit être régulièrement nettoyé ${ }^{6}$. Les cuirs nécessitent également un entretien régulier, avec une cire spéciale (la cire 213 commercialisée par la BnF), afin de garder leur souplesse.

Cette cire ne convient pas aux cuirs très dégradés (cuirs épidermés, cassants, pulvérulents, etc.). Par ailleurs, il ne faut pas appliquer directement la cire sur le cuir mais avec un chiffon.

\section{Inspection des collections}

Une inspection régulière des magasins, avec le regard de la conservation préventive, doit être effectuée quotidiennement à l'occasion de la communication, de façon hebdomadaire, du relevé des thermo-hygromètres, et une fois par an pour l'intégralité des collections.

\section{LA CONSERVATION TOUT AU LONG DE LA VIE EN BIBLIOTHÈQUE}

La conservation est un état d'esprit. Il faut l'appliquer à chaque instant de la vie en bibliothèque. Il est important que tous les membres de l'équipe l'acquièrent, soit par des stages de formation continue, soit par des sessions de sensibilisation en interne.

\section{LA CONSULTATION}

Les dégradations physiques les plus fréquentes - en dehors du stockage sont causées par une mauvaise manipulation des documents pendant l'extraction des rayonnages, le transport, la consultation, ou la reproduction.

\section{L'extraction du rayonnage}

Quand un ouvrage est saisi par la coiffe* pour être retiré du rayonnage, celle-ci casse rapidement. Cela s'évite par exemple en passant la main audessus l'ouvrage et en poussant le livre vers soi par la tranche.

6. Voir les fiches pratiques de la BnF : < http://www.bnf.fr/fr/professionnels/conservation.html >. 


\section{Le transport}

Pendant le transport des magasins à la salle de consultation, quand celuici se fait dans des chariots inappropriés, les ouvrages peuvent tomber : au mieux ils sont éraflés, au pire, s'ils tombent sur la couture, celle-ci peut se casser, les plats se détacher...

Il est recommandé d'utiliser des chariots munis de bords protecteurs afin d'assurer la sécurité des documents en déplacement (en U).

\section{La consultation}

Pendant une communication non encadrée, les risques de dégradation sont multiples : livres empilés qui tombent, rupture des coutures si les lecteurs s'appuient sur les livres, traces d'encre... En particulier, l'utilisation de supports mal appropriés aux livres pour la consultation peut occasionner de nombreux dommages à la reliure. Le lutrin traditionnel en bois proche de la verticale est très nocif pour les livres : il tire sur la couture - qui peut casser - en positionnant les feuillets verticalement.

Le lecteur n'est, le plus souvent, pas au fait des principes et contraintes de conservation ; il est donc du ressort du bibliothécaire de l'en informer, voire de le «former » sur le respect des documents, et de lui expliquer les raisons des différentes consignes de manipulation.

Le principe de base de la conservation préventive, lors de la consultation, est de reprendre les différentes sources de fragilité du livre, et de le faire manipuler par le lecteur en conséquence : la mise en œuvre la plus facile, et la plus efficace, est sans doute le futon modulable de soutien, dont les côtés sont enroulés pour arriver à la hauteur de chaque plat, en fonction de la masse des feuillets situés de chaque côté : la couture ne doit pas souffrir, chaque plat doit être soutenu au maximum, et ne pas reposer sur le vide. De même, on ne doit jamais forcer l'ouverture d'un livre. L'utilisation des gants, au moins pour les photographies et les documents précieux, paraît nécessaire, sauf si d'autres dispositifs peuvent être mis en place. 


\section{LA REPRODUCTION}

\section{La reproduction à la bibliothèque}

Les photocopies de documents patrimoniaux posent de graves problèmes de conservation. Les photocopieurs habituels, liés à une mauvaise manipulation, peuvent causer d'importants dommages à la structure des livres (dos cassé, couture cassée, pages cornées...). En outre, la photocopie est une source très importante de lumière et de chaleur.

La meilleure recommandation qu'on puisse faire est d'interdire la photocopie de tout élément du patrimoine écrit.

Les photocopieurs/numériseurs à prise de vue zénithale, sans besoin de retourner l'ouvrage, sont une solution. Les photocopieurs à pan coupé évitent les dommages mécaniques, mais pas ceux causés par la lumière et la pollution.

\section{La numérisation}

L'impact des campagnes de numérisation sur la conservation des fonds patrimoniaux n'est pas négligeable. La numérisation peut étendre de façon significative les risques de dégradation si les appareils et les processus ne sont pas adaptés à l'exigence première de la conservation.

Les principaux points à surveiller sont l'exposition à la lumière durant la numérisation (à limiter au maximum), et les matériels en contact avec le document lors de l'opération (supports, poids...), qui doivent être recouverts de matériaux de conservation.

\section{LES EXPOSITIONS}

Les conditions de conservation à (faire) respecter lors des expositions de documents graphiques ont fait l'objet d'une norme AFNOR NF Z 40-010 publiée en juin 2005. Nous y renvoyons le lecteur. Nous n'en évoquerons ici que les principaux éléments.

Les expositions accentuent tous les risques que les conditions climatiques et l'éclairage font courir aux documents car l'éclairage est obligatoire, les portes sont ouvertes et la présence humaine forte, rendant difficile le maintien de valeurs climatiques stables ; les documents sont présentés ouverts et donc plus réceptifs à l'action de la lumière et des variations 
thermo-hygrométriques ; les contraintes de présentation vont à l'encontre des principes de conservation : ouverture, inclinaison des documents...

\section{Avant l'exposition}

La démarche de conservation doit être mise en œuvre avant même l'exposition. Il s'agit de l'examen de la possibilité d'exposition pour le document, en fonction de son état physique, et de ses précédentes séances d'exposition ; il s'agit aussi et surtout de la rédaction d'un constat d'état détaillé à 4 étapes (avant/après le transport aller, après l'exposition, au retour dans les magasins), afin de pouvoir déterminer, au cas où une dégradation se ferait jour, le moment précis où celle-ci est intervenue, et la responsabilité.

\section{Pendant l'exposition}

\section{Vitrines et supports}

Les vitrines doivent être étanches à la poussière, fermées avec un élément unique (clé ou ventouse) et de préférence en verre feuilleté.

Les supports doivent être adaptés aux documents (et non l'inverse), ne leur imposer aucune contrainte (angle d'ouverture à $90^{\circ}$ ou $120^{\circ}$ maximum pour les reliures anciennes), soutenir leurs plats et leur couture. Les pages seront maintenues ouvertes par des clips, ou des bandes en polyester. Les documents isolés posés directement au fond des vitrines en seront isolés par une feuille de papier ou carton neutres.

\section{Les conditions thermo-hygrométriques}

Il est donc indispensable de maintenir le document dans des conditions thermo-hygrométriques stables depuis sa sortie de magasin jusqu'à son retour. Les conditions climatiques seront donc contrôlées à l'intérieur de la vitrine, en veillant à refuser toute présence de source de chaleur influant sur la température de la vitrine (source lumineuse, générateur...). Des matériaux tampons stockés en attente dans les mêmes magasins que les ouvrages pourront restituer dans la vitrine le même taux d'humidité relative. 


\section{L'éclairage}

L'intensité d'éclairage se mesure par le lux*, qui est la quantité de lumière reçue par une surface donnée. La lumière naturelle " normale " s'évalue dans une fourchette entre 50000 et 150000 lux.

La lumière a un effet cumulatif sur le document, c'est-à-dire que chaque période où le livre est exposé à la lumière et ouvert à une page donnée s'additionne avec la précédente et la suivante. Il est donc très important de savoir combien de temps un document a été éclairé, et à quelle intensité. Rappelons que le rayonnement visible est aussi nocif que les UV pour l’intégrité des œuvres.

Pour un document, les risques d'être exposé trop longtemps ou trop souvent à la lumière sont nombreux : perte de fraîcheur des couleurs, perte de texte, à terme perte des couleurs elles-mêmes, perte de résistance mécanique, accélération du vieillissement. De plus, les effets de la lumière sur un document sont irréversibles. Enfin, les réactions chimiques qui se déclenchent lors de l'exposition à la lumière se poursuivent, une fois celle-ci éteinte.

En fonction des matériaux les constituant ainsi que de leur état de conservation, les documents de bibliothèque sont répartis en trois catégories suivant leur sensibilité à la lumière : il ne faut pas dépasser la dose totale d'exposition (DTE).

\section{TABLEAU SENSIBILITÉ DES TYPES DE DOCUMENT À LA LUMIÈRE}

\begin{tabular}{|c|c|c|}
\hline $\begin{array}{l}\text { classe de } \\
\text { sensibilité }\end{array}$ & Types de document & DTE / an \\
\hline $\begin{array}{l}\text { Fortement } \\
\text { sensible }\end{array}$ & $\begin{array}{l}\text { Papier pâte chimique ; papier pâte chiffon ; encre } \\
\text { noire d'imprimerie, encres noires manuscrites, } \\
\text { graphite ; tirages photographiques noir et blanc sur } \\
\text { papier baryté ; documents imprimés en bon état }\end{array}$ & $\begin{array}{l}84000 \\
\operatorname{lxh} / \mathrm{a}\end{array}$ \\
\hline $\begin{array}{l}\text { Très fortement } \\
\text { sensible }\end{array}$ & $\begin{array}{l}\text { Parchemin ; tirages noir et blanc } \\
\text { sur papier plastifié }\end{array}$ & $\begin{array}{l}42000 \\
\operatorname{lxh} / \mathrm{a}\end{array}$ \\
\hline $\begin{array}{l}\text { Extrêmement } \\
\text { sensible }\end{array}$ & $\begin{array}{l}\text { Papier pâte mécanique, papier calque, papier } \\
\text { pelure ; encres de couleur, enluminures, } \\
\text { feutres, aquarelles, pastels, gouaches ; tirages } \\
\text { photos couleurs, tirages photos XIX }{ }^{\mathrm{e}} \text { siècle }\end{array}$ & $\begin{array}{l}12500 \\
\operatorname{lxh} / \mathrm{a}\end{array}$ \\
\hline
\end{tabular}


L'action de dégradation sera la même si on expose des documents sous un éclairement de 50 lux durant 250 heures tous les ans, que sous 150 lux durant 250 heures une fois tous les 3 ans.

Il faut donc calculer : si 150 lux sont relevés au luxmètre, et si la durée de l'exposition est de 45 heures par semaine pendant 7 semaines : $150 \times 45$ x $7=47250$ lxh seront reçus par les documents. S'il s'agit de manuscrits sur parchemin enluminés, de périodiques $d u \mathrm{XIX}^{\mathrm{e}}$ siècle, de tapuscrits à l'encre violette $\mathrm{du} \mathrm{xx}^{\mathrm{e}}$ siècle, c'est une durée 3 fois trop longue. Il faut alors soit réduire la durée de l'exposition, soit réduire l'intensité d'éclairage, pour que le résultat du calcul final soit conforme à la DTE correspondant au type de document. On peut aussi prendre des dispositions pour que ces documents ne soient jamais remis en exposition pendant les 3 années suivantes (le noter sur la boîte de conservation par exemple ou la fiche individuelle du document, de façon à savoir ensuite si on peut ou non le ré-exposer et dans quel délai).

Pour ne pas dégrader les documents lors des expositions, on conseille donc de protéger au maximum les documents de la lumière naturelle, de changer régulièrement la page exposée, et de privilégier les lumières froides (fibres optiques), qui évitent les problèmes de chaleur (mais pas ceux de la lumière). Les éclairages dynamiques, qui ne se déclenchent qu'en présence des visiteurs, sont également une bonne solution. Et bien sûr, il ne faut jamais exposer en permanence des documents originaux à vocation de conservation.

\section{LE PLAN D'URGENCE*}

\section{Les risques}

Le feu est généralement considéré comme le fléau naturel des bibliothèques ; s'il survient moins souvent que l'inondation, ses conséquences sont toujours graves s'il n'a pas été combattu dès le début.

Les inondations sont très fréquentes en bibliothèque : dysfonctionnement de plomberie, fuites diverses, orages violents... Les dommages de l'eau sur les documents sont importants : gonflement des papiers faisant éclater les coutures, adhérence des feuillets entre eux, poids accru faisant 
s'effondrer les étagères, taches sur les cuirs, et surtout risque de développement très rapide de moisissures dès que l'humidité relative dépasse $62 \%$. Les conséquences sont alors très lourdes : au mieux il faudra organiser le dépoussiérage complet des collections, au pire la désinfection des documents et des locaux.

\section{La rédaction du plan d'urgence}

Il est indispensable de préparer un plan d'urgence ${ }^{7}$ au minimum pour les collections patrimoniales, au mieux pour l'ensemble des collections, et de former les équipes à la réaction, aux bons gestes à tenir en cas de sinistre. Ce plan d'urgence aura 3 objectifs :

- sensibilisation du personnel aux sinistres ;

- prévention : tout faire pour éviter que le sinistre n'arrive ;

- prévision : tout préparer pour minimiser les conséquences du sinistre s'il survient quand même.

Le plan d'urgence consiste également à :

- travailler avec les pompiers pour leur montrer les locaux, les collections à sauver en priorité ; et pour les sensibiliser au problème des collections graphiques face aux eaux d'extinction...;

- faire une liste des documents les plus précieux à sauver en priorité et convenir avec les pompiers d'un mode de repérage simple, sans ambiguïté et visible malgré la fumée ;

- préparer du matériel pour les interventions d’urgence.

\section{La prévention des sinistres}

\section{Prévention des incendies}

Il est bien sûr interdit de fumer dans l'ensemble des locaux de la bibliothèque. Le local fumeur, qui sera aménagé, sera équipé de cendriers contenant du sable.

7. Pour toutes ces questions et les fiches techniques de la BnF rassemblées, voir < http://www. bouclier-bleu.fr/ >. 
Il ne faut laisser de façon permanente aucun appareil électrique branché (radiateur, cafetière, bouilloire...) dans les locaux (bureaux, salles de repos...).

Il existe différents systèmes de détection incendie. Les plus efficaces combinent une détection des fumées avec une détection des températures élevées. Sauf contre-ordre, le système de détection met en route un système de mise en sécurité ou d'extinction (fermeture des portes coupe-feu, ouverture des issues de secours, envoi du produit d'extinction).

Il existe également plusieurs systèmes d'extinction : les sprinklers, les gaz inertes, ou les brouillards d'eau. Ces derniers sont probablement ceux qui allient le plus efficacité et conservation.

\section{prévention des inondations ou des conséquences d'une inondation}

Il faut prendre l'habitude de signaler tout dysfonctionnement de plomberie, et alerter immédiatement à toute découverte de fuite, éponger et nettoyer tout de suite pour éviter l'extension de l'inondation.

ENCADRÉ

\section{LES INTERVENTIONS EN CAS D'INONDATION} (ET EN PHASE POST-INCENDIE)

- couper l'arrivée d'eau si c'est possible ;

- établir le constat d'état : la première chose à faire est le tour des locaux afin de voir quelle surface est affectée par l'inondation. Faire un croquis (très succinct !), sur des plans vierges préparés, des emplacements précis des magasins où se trouve l'eau : cela peut être important pour déterminer les causes de l'inondation. Dans une description rédigée sommairement, noter les éventuelles différentes hauteurs d'eau ;

- prendre des photos : il est important de penser à prendre des photos du sinistre (vues d'ensemble et de détails). Elles seront très utiles, pour les assurances, et pour aider les services techniques au diagnostic des causes du sinistre ;

- protéger, à l'aide de grandes bâches, les rayonnages qui n'ont pas été atteints si la cause de l'inondation n'est pas réglée ;

- aspirer l'eau avec un aspirateur à eau ou un refouleur: chaque demi-heure compte pour le développement des moisissures ;

- donner priorité au sauvetage des livres inondés ou humides : les livres brûlés pourront attendre quelque temps : leur état ne s'empirera pas au fil des jours ;

- opérer un tri : séparer les livres mouillés (l'eau en ruisselle) des livres secs et des livres humides ; 
- vider les rayonnages, si possible, en respectant l'ordre numérique des cotes, au moins par tranche ;

- agir vite et bien pour les documents mouillés ou humides : des déformations commencent à apparaître au bout de 8 heures, les moisissures en 48 heures ; l'attente occasionnera des dommages irréversibles. Les livres fort mouillés (plats gonflés, dos concaves) seront traités en priorité ;

- privilégier l'intervention sur les locaux de stockage (de préférence aux bureaux et couloirs) de livres à vocation de conservation (patrimoine, fonds régional, dépôt légal...) ;

- aérer les locaux.

\section{LA RESTAURATION}

La restauration occupe le dernier maillon et le plus périlleux de la chaîne de la conservation : une mauvaise restauration peut faire disparaître à jamais des pans entiers de l'identité d'un document.

La restauration est l'action de stopper les dégradations d'un document, en le consolidant dans un état fonctionnel, tout en respectant son intégrité ; de lui donner les moyens de transmettre son témoignage chargé d'histoire. Le but de la restauration est de ralentir le vieillissement inéluctable du document, mais pas d'effectuer un retour en arrière à l'état d'origine.

Il est important d'abord de :

- commencer par agir sur les conditions de conservation de l'ensemble des collections ;

- procéder à l'évaluation de l'état physique des collections ;

- dépenser les premiers crédits en préservation (conditions climatiques, lumière, mobilier, conditionnements, formation du personnel, etc.).

\section{Les modalités : les principes de base}

- La restauration doit s'inclure dans une méthodologie d'ensemble de conservation. On ne restaure pas des ouvrages quand, après restauration, ils reprendront place dans de mauvaises conditions de conservation, climatiques ou de stockage ;

- tous les ouvrages dégradés ne doivent pas nécessairement passer par la restauration : une sélection s'impose ; 
- le plus souvent, il faut s'en tenir à une intervention minimale, limitée à ce qui est strictement nécessaire à la consultation des documents ;

- les matériaux doivent être compatibles avec les matériaux d'origine ;

- les opérations doivent être réversibles : chaque élément apporté doit pouvoir être démonté et retiré sans autrement altérer les éléments d'origine ;

- l'intervention doit être honnête, transparente et discrète sur le plan esthétique.

Les critères de choix des ouvrages à diriger vers la restauration devront prendre en compte :

- des ouvrages qui sont consultés ;

- des ouvrages avec particularités d'exemplaire (ex-libris*, ex-dono*, reliure aux armes, annotations manuscrites...) ;

- des ouvrages pour lesquels la valeur documentaire ou artistique, d'intérêt local, régional ou national, est importante ;

- des ouvrages dont les dégradations sont de nature à en empêcher la consultation, à s'étendre et endommager davantage le document au fil des années et des consultations (par exemple, couture cassée, feuillets désencollés ou devenus mous par l'action de l'eau...).

La sélection des ouvrages à restaurer doit être effectuée non pas par le restaurateur mais par le responsable du fonds. Si celui-ci ne se sent pas capable de le faire, des stages de formation continue sont accessibles ; sinon, il vaut mieux alors ne pas engager d'opérations de restauration.

\section{Le dossier de restauration}

Un dossier de restauration doit accompagner le livre pendant la restauration, être complété en cours de travail, regagner la bibliothèque avec le livre, et être conservé sinon avec le livre, dans tous les cas aussi longtemps que celui-ci. Il est le carnet de santé de l'ouvrage. Un document doit garder une évocation écrite de son histoire.

Ce dossier comprend six grandes parties et 2 séries de photographies : 
- l'identification du document ;

- des photographies nombreuses et nettes de tous les aspects dégradés ;

- la description des éléments permettant de déceler l'état original ;

- la description de l'état de conservation ;

- le projet de restauration ;

- le contre-projet, le devis et la fiche technique ;

- le compte rendu de restauration ;

- les photographies de l'ouvrage après la restauration.

Les dossiers doivent être soumis au CTR, via le SLL au ministère de la Culture et la Communication ${ }^{8}$ : c'est obligatoire pour les documents appartenant à l'État et conservés en bibliothèque municipale, recommandé pour les autres. 


\section{PARTIE II}

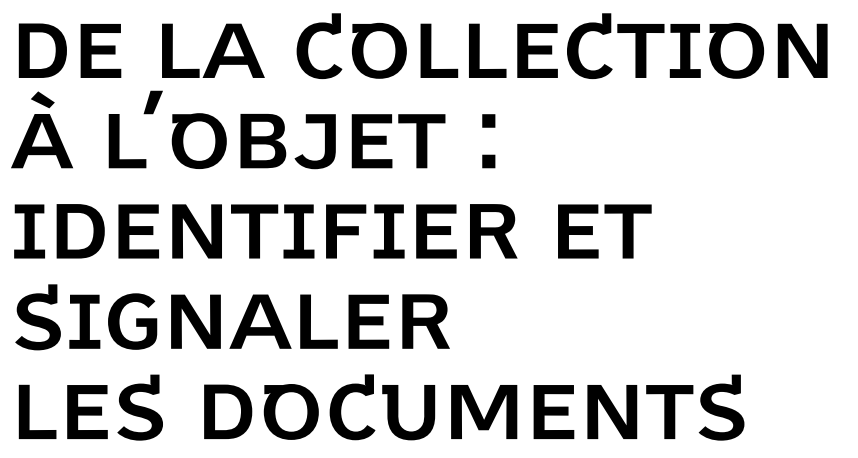

1. LES LIVRES ANCIENS : FORMATS, CAHIERS, SIGNATURES, PAGE DE TITRE, FAUSSES ADRESSES, COLOPHON, ET TOUTES CES SORTES DE CHOSES...

par Dominique Coq

2. RELIURES FRANÇAISES SOIGNÉES ET COURANTES (MI-XV ${ }^{\mathrm{e}}$ SIĖCLE - XIX ${ }^{\mathrm{e}}$ SIÈCLE), ÉLÉMENTS D'IDENTIFICATION par Fabienne Le Bars

3. ESTAMPES : COMMENT IDENTIFIER LES TECHNIQUES? par Séverine Lepape

4. COMMENT IDENTIFIER LES PRINCIPALES TECHNIQUES PHOTOGRAPHIQUES?

par Patrick Lamotte

$+++++++++++++++++++++++++++++++++++++++++++++++$

5. DÉCRIRE ET SIGNALER DES DOCUMENTS PATRIMONIAUX :

ENJEUX, FORMATS, PERSPECTIVES

par Florent Palluault 


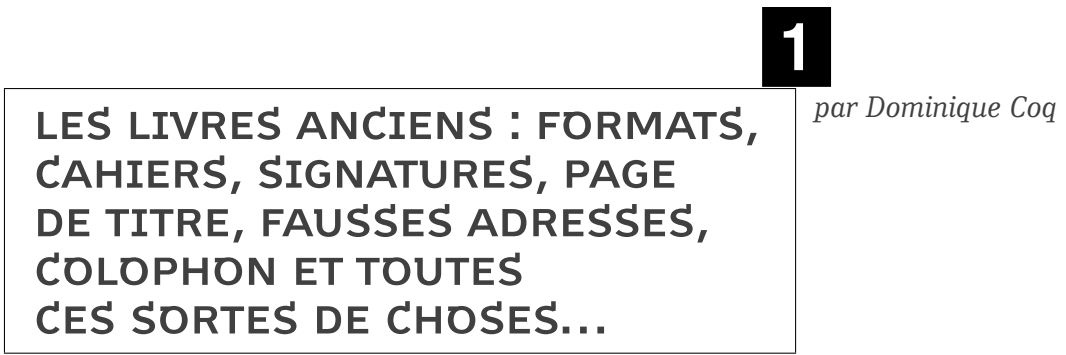

Vous avez pris connaissance de ce qui fait la nature d'une collection patrimoniale, de son statut et de la manière de la conserver. Examinons maintenant les différents types de document qui la constituent et la manière de les cataloguer.

Tout d'abord, les livres qui forment naturellement la grande majorité des objets qu'une bibliothèque conserve dans sa collection patrimoniale. Qu'ils soient écrits à la main (manuscrits) ou produits par la typographie (imprimés), ces livres ont une structure qui n'a quasiment pas changé depuis la fin de l'Antiquité : le codex, objet parallélépipédique constitué d'un ensemble de cahiers formés de bifeuillets* (ou feuillets* conjoints) encartés (emboîtés) les uns dans les autres et reliés ensemble par un même passage du fil de reliure.

Je n'évoquerai dans ce chapitre que le cas des livres anciens (en gros imprimés antérieurement au XIX ${ }^{\mathrm{e}}$ siècle) et ce qu'il faut relever dans la notice catalographique les concernant. Pour les manuscrits, je renvoie à deux excellents documents en ligne auxquels on peut, le cas échéant, se référer :

- pour les manuscrits médiévaux, le livret du stage d'initiation au manuscrit médiéval mis en ligne par l'Institut de recherche et d'histoire des textes $\left(\right.$ IRHT) ${ }^{1}$;

- pour les manuscrits modernes et contemporains, la recommandation de l'AFNOR concernant la description des manuscrits et fonds d'archives modernes et contemporains en bibliothèque : DeMArch ${ }^{2}$.

1. < http://www.irht.cnrs.fr/sites/all/files/Livret_stage_2011b.pdf >.

2. < http://www.bivi.fonctions-documentaires.afnor.org/livres-blancs/demarch-recommandationde-description-des-manuscrits-et-fonds-d-archives >. Voir plus loin p. 124. 


\section{IDENTIFIER L'ÉDITION}

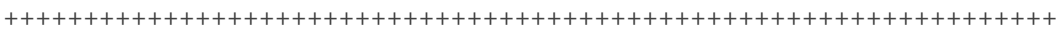

L'identité d'un livre ancien comporte un certain nombre d'informations à relever dans la notice descriptive.

\section{L'AUTEUR ET LE TITRE}

L'auteur de l'ouvrage et le titre sont généralement faciles à trouver à la page de titre, comme pour un livre moderne. Mais pour les éditions les plus anciennes (disons jusqu'en 1520 /1530) c'est plus compliqué : comme pour la plupart des manuscrits médiévaux, elles commencent par un incipit (les premiers mots du texte) dans lequel sont indiquées - ou non - les mentions de titre et d'auteur. Mais ces informations sont aussi à trouver à l'explicit de l'ouvrage (les dernières lignes du texte), qui peut donner des indications sur la date et les circonstances de la rédaction de l'ouvrage. $\mathrm{Ne}$ pas confondre avec le colophon que l'on verra plus loin.

\section{L'ADRESSE TYPOGRAPHIQUE}

On trouve le plus souvent à la page de titre les informations concernant le lieu d'impression, l'imprimeur ou le libraire, et la date d'édition. Dans la notice il est préférable de traduire dans la langue vernaculaire actuelle les données qui sont assez souvent indiquées en latin. On trouvera la traduction des noms de ville, par exemple, dans l'Orbis latinus de Graesse en ligne $^{3}$. Les dates sont le plus souvent exprimées en chiffres romains.

- Les éditions les plus anciennes ne fournissent pas forcément les informations concernant l'adresse typographique et, quand elles les donnent, c'est au colophon situé à la fin du texte (pas forcément à la fin du volume car des annexes et des tables peuvent suivre l'ouvrage proprement dit). Plus de la moitié de ces éditions étant dépourvues de colophon, laissons aux spécialistes le soin de les dater et d'en identifier l'imprimeur.

- Il n'est pas rare que des éditions des $\mathrm{XVII}^{\mathrm{e}}-\mathrm{XVIII}{ }^{\mathrm{e}}$ siècle soient munies de fausses adresses, souvent parce qu'il s'agissait de livres prohibés ou de contrefaçons. Parmi celles que vous risquez de rencontrer le plus souvent se trouve celle de "Cologne, Pierre Marteau ».

3. < http://www.columbia.edu/acis/ets/Graesse/contents.html >. 


\section{COLLATION}

S'agissant du format d'un livre ancien, on ne parle pas comme aujourd'hui de sa hauteur exprimée en centimètres, mais de son format réel, ou bibliographique, résultant du mode de pliage des feuilles qui le constituent.

Les feuillets évoqués plus haut résultent en effet du pliage d'une pièce rectangulaire de papier ou de parchemin appelée feuille. La façon dont cette feuille de départ (in-plano) est pliée $\mathrm{n}$ fois en deux pour former $2^{\mathrm{n}}$ feuillets détermine le format selon le tableau ci-dessous.

\section{TABLEAU LES FORMATS DE BASE}

\begin{tabular}{|c|c|c|}
\hline Feuille pliée & Nombre de feuillets & Format obtenu \\
\hline 1 fois en deux & $2^{1}=2$ feuillets & in- $\mathrm{f}^{\circ}$ (in-folio) \\
\hline 2 fois en deux & $2^{2}=4$ feuillets & in-4 (in-quarto) \\
\hline 3 fois en deux & $2^{3}=\mathbf{8}$ feuillets & in- $8^{\circ}$ (in-octavo) \\
\hline 4 fois en deux & $2^{4}=16$ feuillets & in-16 (in-seize) \\
\hline 5 fois en deux & $2^{5}=32$ feuillets & in-32 (in-trente-deux) \\
\hline
\end{tabular}

Ce sont les formats de base. Mais il y en a d'autres plus complexes, en particulier l'in-12, fréquemment utilisé dans les livres du $\mathrm{XVII}^{\mathrm{e}}$ et $\mathrm{XVIII}^{\mathrm{e}}$ siècles, qui ont des cahiers uniformément de 12 feuillets ou alternativement de 8 et 4 feuillets.

Pour identifier à coup sûr le format d'un livre ancien sur papier, divers indices doivent être pris en compte :

- la position de certaines traces que le feuillet révèle quand on l'examine par transparence. Ici un bref rappel de la fabrication du papier est nécessaire. En gros, les feuilles de papier - je parle du papier ancien dit "papier à la forme » qui fut exclusivement utilisé jusqu'au début du XIX ${ }^{\mathrm{e}}$ siècle - proviennent du défibrage par trituration mécanique de morceaux de textiles appelés chiffe dans de l'eau très pure. La pâte obtenue est versée et étalée sur un tamis rectangulaire (la forme) formé d'un réseau de fils de laiton accrochés à un cadre de bois : les vergeures, disposées de manière serrée parallèlement au grand côté, que croisent pardessous les lignes de chaînettes (appelées communément pontuseaux), 
plus espacées, parallèles au petit côté. Au milieu d'une des moitiés de la forme le papetier fixe généralement un motif de laiton, le filigrane, qui est sa marque de fabricant. L'eau s'écoule à travers les fils comme à travers un crible et la pâte reste, formant sur le fond de la forme une couche mince que l'on fait sécher. Les feuilles de papier obtenues après séchage et encollage montrent par transparence les empreintes des pontuseaux et des vergeures ainsi que du filigrane. La disposition des pontuseaux change donc à chaque fois qu'on plie et replie la feuille :

Verticale au départ sur la feuille in-plano et à son premier pliage par le milieu du grand côté (in-folio), la disposition des pontuseaux devient horizontale dans l'in- $4^{\circ}$, puis à nouveau verticale dans l'in- $8^{\circ}$, et ainsi de suite à chaque repliage (voir schéma p. 75).

- Ne jamais chercher la disposition des pontuseaux dans les gardes : ces feuillets de protection ajoutés en tête et en queue de l'ouvrage lors de son travail par le relieur ne font pas partie de l'édition proprement dite.

- La position du filigrane est un indice complémentaire du format : au milieu d'un feuillet dans l'in-folio, au milieu de la marge de pli dans l'in- $4^{\circ}$, dans un coin externe du feuillet dans l'in- $8^{\circ} 4$;

- la dimension des feuillets, d'autant plus réduite que la feuille initiale a été davantage pliée.

Avec le perfectionnement des techniques papetières, la taille des feuilles est passée d'une hauteur d'environ $50 \mathrm{~cm}$ vers 1500 à $90 \mathrm{~cm}$ au $\mathrm{XIX}^{\mathrm{e}}$ siècle. Ainsi, un in- $4^{\circ} \mathrm{du} \mathrm{XVI}^{\mathrm{e}}$ siècle peut-il être plus petit qu'un in- $8^{\circ} \mathrm{du} \mathrm{XIX}{ }^{\mathrm{e}}$ siècle.

- le nombre de feuillets (toujours pair car chaque feuillet a son conjoint en principe) dans un cahier est un indice complémentaire, surtout pour

4. Pour de plus amples informations, voir l'article de Jeanne Veyrin-Forrer, " Fabriquer un livre $\mathrm{au} \mathrm{XvI}^{\mathrm{e}}$ siècle », dans Histoire de l'édition française, sous la dir. de Henri-Jean Martin et Roger Chartier, T. I, $2^{e}$ éd., Paris, Fayard et Éditions du Cercle de la Librairie, 1989, pp. 336-369. 
les petits formats : 8 pour l'in- $8^{\circ}, 12$ ou $8+4$ pour l'in-12, 16 pour l'in16 , etc. Pour les in-folio et les in- $4^{\circ}$, c'est plus compliqué car il était nécessaire d'encarter* plusieurs feuilles par cahier afin que chaque cahier ait un nombre suffisant de feuillets pour résister à la couture. Ainsi il est très fréquent que les volumes in-folio, surtout au $\mathrm{xv}^{\mathrm{e}}$ et $\mathrm{xvI}^{\mathrm{e}}$ siècles, contiennent 8,10 voire 12 feuillets par cahier, ou que des in- $4^{\circ}$ en comptent 8.

On ne retient pas dans ce comptage les éventuels feuillets supplémentaires des planches gravées en taille-douce*5, qui sont imprimés indépendamment du texte typographié.

\section{Repérer les cahiers du volume}

Mais comment allez-vous reconnaître où commencent et finissent les cahiers dans un volume relié ? Grâce à des systèmes de repérage que les typographes (à la suite des copistes) ont adoptés pour indiquer aux relieurs l'ordre dans lequel il fallait relier les cahiers du volume. Les deux principales sont les suivantes :

- les signatures, généralement imprimées dans le coin inférieur du recto des feuillets : elles consistent en une lettre suivie, sauf pour le premier feuillet, d'un chiffre romain ou arabe. Ces lettres vont de $\mathrm{A}$ à $\mathrm{Z}$ (sans $\mathrm{J}$, U et W). Au-delà de $\mathrm{Z}$, on redouble puis on triple : $\mathrm{Aa}, \mathrm{Bb}$, etc., puis Aaa, Bbb... Seuls les premiers feuillets du cahier, en général la première moitié, portent la signature.

- les réclames : indication, sous le texte du verso d'un feuillet, du premier mot du recto du feuillet suivant, généralement à la jonction de deux cahiers ${ }^{6}$.

5. Voir infra l'article de Séverine Lepape : « Estampes : comment identifier les techniques ?», p. 98 et suivantes.

6. Pour en savoir plus sur la matérialité des livres, consulter < http://dominique-varry.enssib.fr/ bibliographie\%20materielle. >. 


\section{SCHÉMA PLIAGE : LES FORMATS}

1 pontuseaux $\quad 0$ Filigrane entier

\|\| vergeures $\quad \triangle$ Filigrane divisé en 2

pliure intérieure 7 Filigrane divisé en 4
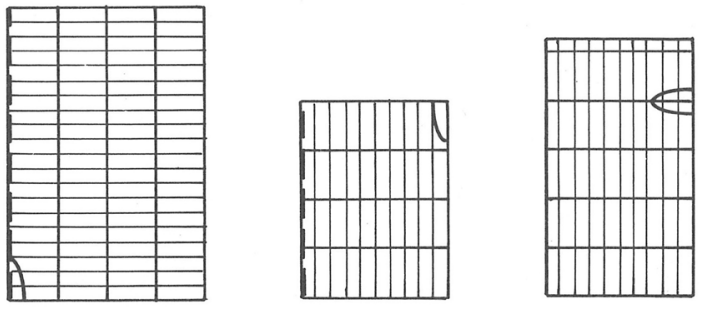

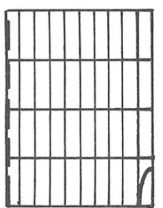

In-16

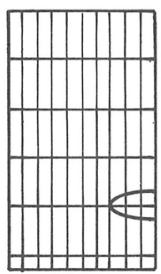

In-12 large $\approx$

$\frac{0}{0}$

ב

ర

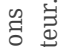

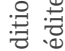

诖

능

륭 몽

敢

क्ष

สี

인

गृं ฮ्త్

ก .

ก ช

تU

ค :

:

式

ઈํํ

ㄹํ

: 을

:

\&

ะ

ㅎำ

运.

ํ. 지

율

艺

ธิ ฮ

In-plano

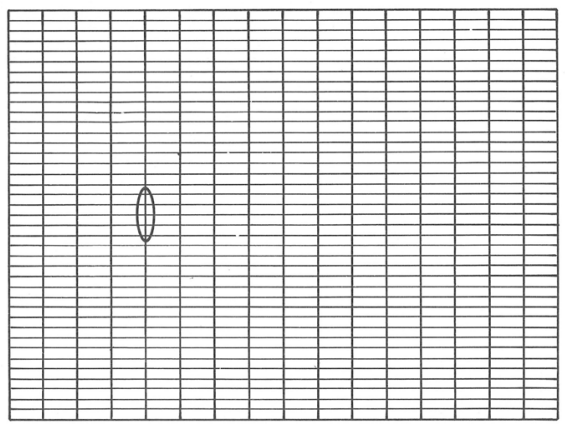

In-folio

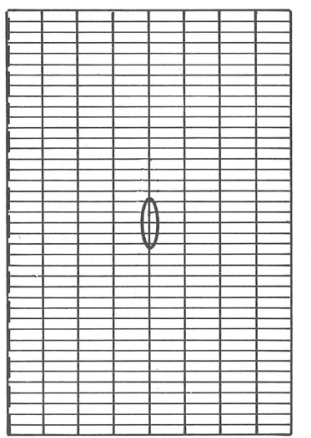

In $-4^{\circ}$

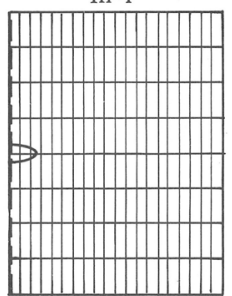




\section{Disposition du texte}

Le texte peut être imprimé " à longues lignes » ou sous deux, voire plusieurs colonnes. La mention éventuelle du nombre de colonnes suit l'indication du format (ex. « in-fol. sous 2 col. »).

\section{Foliotation / pagination}

- La foliotation : il s'agit de la numérotation des feuillets, placée sur leur recto. Elle n'est plus guère en usage après les premières années $d u \mathrm{XVII}^{\mathrm{e}}$ siècle.

- La pagination s'est peu à peu substituée à la foliotation : elle numérote les faces consécutives des feuillets, appelées pages, en haut ou en bas de celles-ci. La numérotation des feuillets et des pages est souvent exprimée en chiffres romains surtout pour les parties liminaires d'un livre, celles qui précèdent le texte de l'ouvrage proprement dit (préface, épître dédicatoire, avertissement, privilège, liste des souscripteurs, etc.). Cette pratique a perduré jusqu'au milieu du $\mathrm{xx}^{\mathrm{e}}$ siècle dans les éditions soignées.

Nota bene :

- Les chiffres romains utilisés dans la foliotation ou la pagination sont souvent imprimés en minuscules, le « i » final d'un nombre étant généralement remplacé par un «j » (ex. ciij pour 103).

- Il est fréquent que les parties en tête ou en fin d'ouvrage (comme les tables par exemple) ne soient ni foliotées ni paginées. Il faut donc en donner le nombre de pages ou de feuillets entre crochets carrés (ex. xij, 192 - [4] pp.).

- Votre exemplaire peut être incomplet de pièces liminaires, d'index ou de tables. Bien vérifier la collation dans les bibliographies ou les catalogues de grandes bibliothèques (comme le catalogue général de la BnF en ligne, par exemple).

\section{L'illustration}

Les images, assez fréquentes dans les livres, n’ont pas toutes le même statut: 
- Les motifs ornementaux (lettrines*, bandeaux*, fleurons*, vignettes*, culs-de-lampe*) ne sont pas dénombrés dans une notice bibliographique de base.

- Les figures gravées sur bois ${ }^{7}$ qui, techniquement, peuvent être imprimées en même temps que le texte typographié, sont incluses dans la pagination et ne sont généralement pas dénombrées mais indiquées dans la collation sous la dénomination générique « fig. » (pour figures).

- Les planches hors texte doivent être dénombrées (ex. 11 pl.).

Comme pour la pagination, il faut vérifier dans les bibliographies et les catalogues si l'exemplaire qu'on a en main comporte bien le nombre requis de planches.

- La marque d'imprimeur ou de libraire bien souvent accompagnée d'une devise, que l'on trouve la plupart du temps à la page de titre, n'est pas une illustration même si elle est imagée. Gravée sur bois ou quelquefois en taille-douce, c'est une marque de fabricant. Il convient d'en signaler l'existence à la fin de la collation.

\section{INDIVIDUALISER L'EXEMPLAIRE}

Après avoir relevé les caractéristiques communes à tous les exemplaires d'une même édition, il faut s'attaquer aux particularités de l'exemplaire qu'on a entre les mains. Longtemps négligées par les bibliothécaires, ce sont parfois les données les plus intéressantes pour le chercheur. Relevées de manière systématique, ces données « locales » permettant de retracer l'histoire d'un exemplaire fournissent souvent des informations précieuses sur le fonds dont il fait partie.

7. Voir infra l'article de Séverine Lepape : « Estampes : comment identifier les techniques ? ", pp. 96-97. 


\section{LE SUPPORT}

Quelques rares exemplaires de luxe d'éditions anciennes ont été imprimés sur du parchemin, voire du vélin ${ }^{8}$, comme beaucoup de manuscrits médiévaux.

\section{LES INCOMPLÉTUDES}

Indiquer, le cas échéant, les feuillets qui manquent dans l'exemplaire. Cela suppose de vérifier avec la collation d'un exemplaire complet dans une bibliographie.

\section{LES MARQUES DE PROVENANCES}

Elles sont de plusieurs sortes :

\section{- Ex-libris}

On trouve fréquemment à la page de titre ou aux contreplats ou sur les gardes des mentions d'appartenance écrites par le possesseur sous des formulations très variées, en français ou en latin. (ex. "Ce livre appartient a moy, Françoys Le Nel, grenetier a Loudun. Qui le trouve me le rende et il boira le vin »; " Ex libris Bibliothecae Patrum Carmelitarum Burdegalensium »). Là encore l'Orbis latinus rend de grands services pour identifier les toponymes en latin.

Mais lorsque le possesseur se trouve à la tête d'une collection de quelque importance, il s'est souvent fait confectionner un ex-libris gravé qu'il appose au contreplat supérieur ou sur une des gardes de ses livres. Cette marque d'appartenance est à relever soigneusement et à identifier dans la mesure du possible. Le nom du propriétaire n’y figure pas forcément mais il peut y avoir mis ses initiales, ses armoiries ou sa devise.

\section{- Ex dono}

Qu'elle soit manuscrite ou gravée, cette marque d'appartenance indique le nom du donateur du volume.

8. Voir la définition de ces termes dans l'article de Fabienne Le Bars : "Reliures françaises soignées et courantes (mi-Xve siècle - XIX ${ }^{\mathrm{e}}$ siècle) : éléments d’identification », p. 83. 


\section{- Reliure aux armes}

Comme pour l'ex-libris gravé, le possesseur pouvait se faire confectionner un ou plusieurs fers à ses armes qui étaient poussés sur les plats et/ ou dans les compartiments du dos de ses livres reliés. Cette pratique a perduré jusqu'au XIX ${ }^{\mathrm{e}}$ siècle. Si l'on a assez de connaissances héraldiques pour blasonner les armoiries le plus souvent anonymes, il est précieux d'avoir recours aux tables héraldiques d'un répertoire comme le Manuel de l'amateur de reliures armoriées françaises ${ }^{9}$.

C'est justement de reliure qu'il va être question dans la contribution suivante, une importante particularité d'exemplaire. Le schéma présenté en détaille les différentes parties.

9. Eugène Olivier, Georges Hermal et Robert de Roton, Manuel de l'amateur de reliures armoriées françaises, Paris, C. Brosse, 1924-1938. 30 vol. + index. 


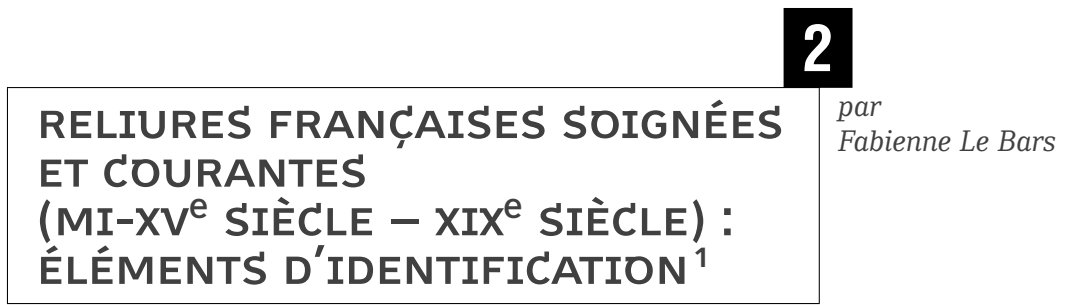

La très grande majorité des livres anciens conservés dans les bibliothèques sont des documents reliés, qu'ils soient ou non dans leur reliure d'origine, tant il est vrai que la fonction première d'une reliure est la protection du livre et sa mise à disposition pour la lecture. Ces reliures, dont la production en France du milieu du XV à la fin du XVIII ${ }^{\mathrm{e}}$ siècle est considérable, offrent des qualités de facture extrêmement variables, déterminées par leur usage et que Jean Toulet a proposé de classer en trois catégories, selon une hiérarchie qui se met en place au cours du XVI ${ }^{\mathrm{e}}$ siècle :

- les reliures de luxe à (grand) décor, réalisées à l'initiative d'un collectionneur ou d'un amateur fortuné ou pour des exemplaires de prestige ;

- les reliures soignées, faites à la demande d'amateurs moins riches mais soucieux de posséder une bibliothèque bien reliée ;

- les reliures courantes, exécutées pour des clients aux attentes plus modestes ou encore pour des libraires.

Les reliures de luxe, très richement ornées, sont celles qui, par leur caractère unique et leur dimension historique, ont suscité le plus d'intérêt et de publications. À côté de ces pièces exceptionnelles, les reliures courantes et soignées, qui constituaient le quotidien des ateliers, ont été peu étudiées, leur importance dans l'histoire socio-économique du livre n'ayant été que plus récemment considérée. Il s'agit donc ici, afin de faciliter l'identification et la description de ces reliures plus modestes, d'en proposer une typologie, qui repose sur un relevé systématique de leurs principales caractéristiques et évolutions au fil des siècles.

1. Pour mieux distinguer les différents procédés, il est conseillé de se reporter à la galerie d'images en ligne qui accompagne ce manuel : <http://www.enssib.fr/presses/galeriebao26 $>$. 


\section{SCHÉMA LES PARTIES CONSTITUTIVES D'UNE RELIURE}

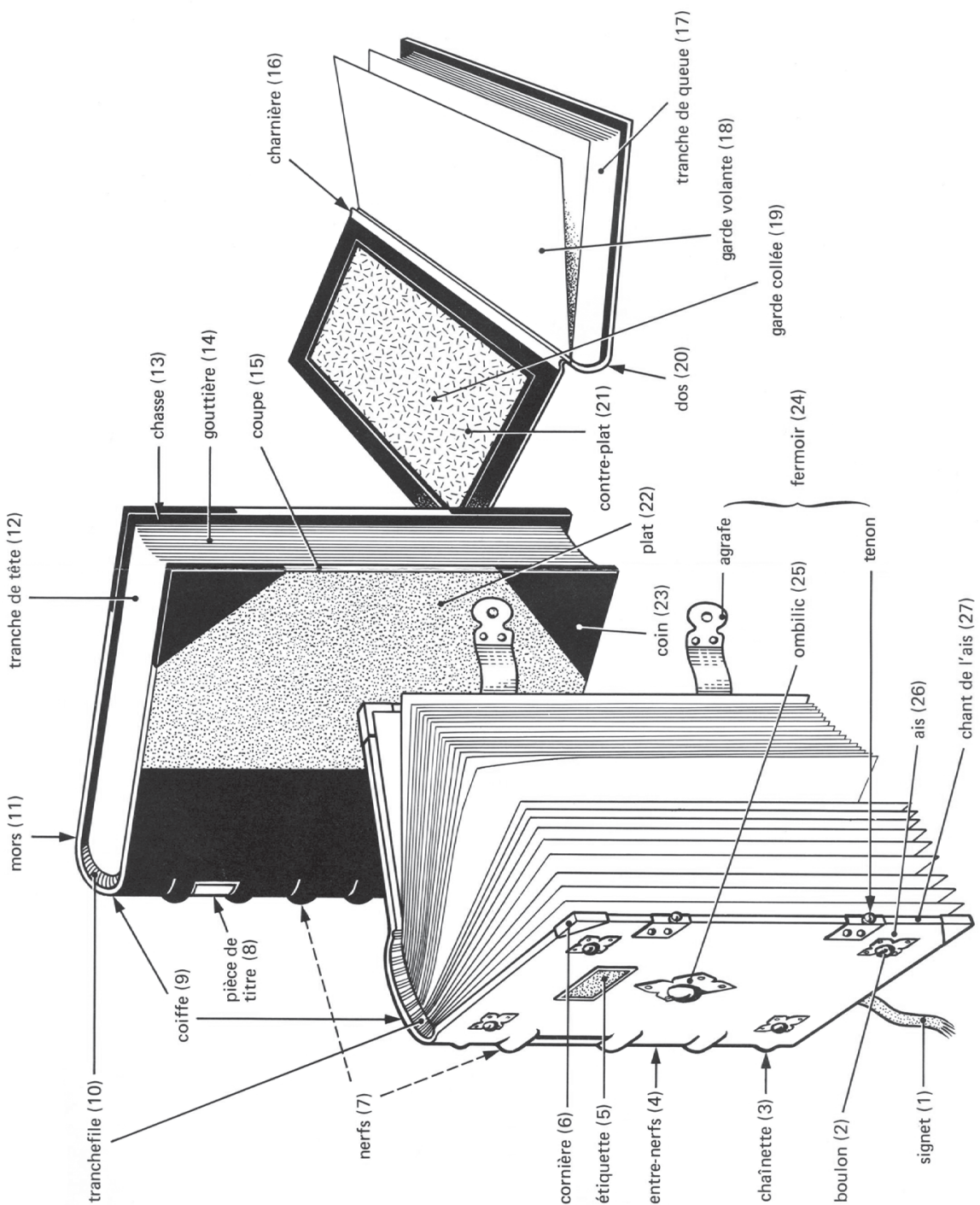

Extrait de : Élisabeth Baras, Jean Irigoin et Jean Vezin, La reliure médiévale : trois conférences d'initiation, Paris, Presses de l'École normale supérieure, 1978, (fig. 31). Reproduit avec l'aimable autorisation de l'éditeur. 
La combinaison de l'ensemble des critères ainsi définis vise à mettre en évidence le mode de fabrication de ces pièces et, par la suite, à apprécier leur place dans l'échelle de production, à les dater et à les localiser. Ces éléments viennent compléter les informations de premier ordre que constituent toujours l'identification de l'édition et, le cas échéant, toutes mentions portées sur l'ouvrage considéré : la reliure existe d'abord pour l'ouvrage qu'elle protège.

Les tableaux ci-après, organisés par principaux éléments constitutifs d'une reliure, mettent en parallèle reliures soignées et reliures courantes. Ils ne prétendent évidemment pas à l'exhaustivité et s'ils offrent un éventail de données suffisamment large pour raisonnablement couvrir les principaux exemples de reliures courantes et soignées réalisées en France à la période moderne, ils ne demandent qu'à être encore enrichis. L'objectif sera atteint s'ils se révèlent un outil pratique à l'usage.

\section{LEXIQUE}

Pour la bonne compréhension du vocabulaire utilisé dans ces tableaux, il a paru utile de définir au préalable un certain nombre de termes et de techniques. Un schéma légendé a aussi été joint ${ }^{2}$.

\section{MATÉRIAUX DE COUVRURE*}

L'identification d'un cuir repose sur la reconnaissance de ses particularités intrinsèques. Lorsque l'hésitation perdure, principalement entre la basane et le veau, le double examen du mode de dégradation du cuir et des pores de la peau permet le plus souvent d'y remédier. Les pores n'étant pas toujours aisément visibles à l'œil nu, le recours à une loupe ou à un compte-fils peut se révéler utile. Rappelons que l'endroit de la peau (du côté des poils de l'animal) est appelé fleur, l'envers (du côté intérieur de l'animal) est appelé chair.

2. Voir supra le schéma « Les parties constitutives d’une reliure », p. 81. 


\section{Reconnaissance des cuirs}

- basane : peau de mouton tannée, plus ou moins lisse et presque sans grain, à pores espacés ; dégradation par usure de la fleur du cuir, abrasée et pelucheuse ;

- maroquin : peau de chèvre tannée, épaisse et plus ou moins lisse, à gros et larges grains réguliers bien visibles;

- maroquin à grain long : peau de chèvre tannée, épaisse et à grain étiré dessinant de fines lignes parallèles en léger relief ;

- parchemin : peau de chèvre ou de mouton (plus rarement de veau) non tannée, lisse et de couleur blanchâtre, traitée par chaulage, écharnage, ponçages et encollages ;

- peau mégissée ou mégie : peau d'animal (sauvage ?) indéterminé, à pores variés, assez épaisse, d'aspect plus ou moins lisse, et blanchâtre ;

- peau retournée : peau d'animal indéterminé, utilisée côté chair et offrant au toucher un contact velouté ;

- truie : peau de porc tannée, très épaisse, de couleur blanchâtre, à pores réunis en forme de triangle ;

- veau : peau de veau tannée, très lisse et presque sans grain, à pores très serrés ; dégradation par éclats de petits morceaux à bords francs ;

- vélin : peau de veau non tannée mais parcheminée, extrêmement fine.

\section{Traitement des cuirs}

Aux $\mathrm{XVII}^{\mathrm{e}}$ et $\mathrm{XVIII}{ }^{\mathrm{e}}$ siècles, de nombreux traitements sont appliqués sur les cuirs de second choix pour dissimuler leur qualité médiocre ou des défauts de fabrication. On peut retenir le terme générique « marbré » pour les désigner, une identification plus fine pouvant être proposée en fonction de leur aspect final ${ }^{3}$.

Techniquement, ces traitements sont obtenus par des mélanges aqueux d'acides mordants (parfois additionnés de couleurs), passés sur les cuirs après couvrure. Les modes opératoires sont les suivants :

- marbrure à l'éponge : applications directes d'une éponge imbibée, sur un livre relié posé à plat ;

3. Voir plus loin les tableaux « Typologie des reliures soignées et courantes », pp. 88-94. 
- marbrure au pinceau : secousses imprimées à un pinceau imbibé et placé au-dessus d'un livre relié dont les plats ont été relevés sur des tringles. Ces deux procédés (à l'éponge ou au pinceau) peuvent être associés ;

- jaspure : passage d'une brosse dure imbibée sur une grille, placée au-dessus d'un livre relié, disposé comme ci-dessus.

\section{ÉLÉMENTS STRUCTURELS}

Ces éléments sont particulièrement observables lorsque la couvrure de la reliure est lacunaire.

\section{couture}

La couture désigne l'assemblage des cahiers d'un volume, réunis par passage d'une aiguillée de fil ; l'entrecroisement de ce fil en tête et queue du dos, lors du passage d'un cahier à l'autre, est appelé chaînette (car évoque un maillon de chaîne).

On distingue trois catégories de couture :

\section{Couture sur nerfs}

Les trois composants d'une couture sur nerfs sont :

- la couture proprement dite ; la plus fréquente est la couture à chevrons, ainsi désignée pour la forme de $\mathrm{V}$ que dessine le passage du fil de couture ;

- l'âme de la couture, autrement dit son support : il s'agit des nerfs, protubérances au dos des cahiers autour desquels s'enlace le fil de couture ; ils assurent, par leur prolongement, la cohésion du bloc de cahiers* avec les plats ;

- la nature du matériau de l'âme : nerfs de peau, de cuir ou de ficelle (dite aussi septain car faite à l'origine de sept brins roulés).

La couture sur nerfs détermine l'aspect final du dos de la reliure après couvrure : l'emplacement et le nombre de nerfs visibles sont l'exact reflet de la structure interne. 


\section{Couture grecquée}

Elle se caractérise par l'absence de nerfs visibles, les ficelles supportant le fil de couture étant logées dans des entailles préalablement opérées au dos des cahiers au moyen d'une scie, dite à grecquer.

La couture grecquée ne détermine pas l'aspect final du dos de la reliure. Une carte à dos, fixée au niveau des mors*, est intercalée entre le dos des cahiers et le matériau de couvrure, selon une technique dite à dos brisé (le cuir n'est pas collé directement au dos des cahiers et la carte à dos s'en désolidarise à l'ouverture du volume). Si la carte est laissée telle, le dos de la reliure est long, autrement dit sans relief ; si de fines bandes de cuir ou de carton (dites faux-nerfs) sont collées sur la carte, le dos de la reliure est à nerfs, à l'identique d'un dos à véritable couture sur nerfs.

\section{Couture par surjet}

Elle a pour but de constituer en cahier des feuillets simples, par la réalisation d'un point dit de surjet, réalisé en biais et en chevauchant les bords des feuillets. Elle est principalement utilisée pour les recueils de planches. Ces cahiers sont ensuite réunis par une couture sur nerfs ou grecquée.

\section{Renforts}

Les renforts sont des éléments de consolidation apportés au niveau du dos et des charnières des volumes, les zones les plus fragiles d'une reliure car directement sollicitées lors de la manipulation du volume. On distingue :

- la claie, bande en parchemin collée dans les entrenerfs du dos et prolongée de manière à être contrecollée sur les contreplats ;

- la charnière, bande en parchemin placée tout au long dans le mors intérieur, qui constitue la zone d'articulation entre le plat et le dos ; elle est généralement collée au contreplat mais laissée libre du côté du corps d'ouvrage.

On note aussi l'usage de bandes de parchemin ou de papier (souvent des défets*) collées sur la largeur de l'entrenerf, sans prolongement. Cet élément s'apparente plus, surtout lorsqu'il est de papier, à un apprêt destiné à lisser les imperfections du dos avant la couvrure (opération d'apprêture). 


\section{TRANCHEFILES*}

La tranchefile est l'élément qui renforce ou soutient les deux extrémités du dos, au niveau des coiffes. Elle est généralement définie par trois éléments :

- le bâti : mode de fixation au corps d'ouvrage (essentiel au Moyen Âge) ;

- l'âme : support de cuir ou de ficelle destiné à recevoir la broderie ;

- la broderie : fils assemblés avec des points d'une grande variété.

Les principaux types rencontrés en France du milieu du $x v^{e}$ siècle à la fin du XvIII $^{\mathrm{e}}$ siècle sont les tranchefiles :

- à passe : fil brodé sur une lanière de peau ou un septain fixé aux ais ou cartons ;

- simples : fil brodé à point vertical sur un septain ;

- à chapiteau : fil brodé sur un double niveau ;

- rapportées : petite ficelle ou papier roulé enveloppé dans un tissu.

\section{GARDES DE COULEURS}

Les gardes de papier blanc, dont il convient de toujours relever la présence éventuelle de filigranes, sont de règle jusqu'au milieu du XvII ${ }^{\mathrm{e}}$ siècle, époque à laquelle sont aussi peu à peu introduites les contregardes puis les gardes volantes de couleurs.

Ces papiers colorés, aux motifs d'une infinie variété, peuvent être classés en six catégories principales, selon leur technique de fabrication :

- papier à la colle : application d'un mélange de colle et de couleurs ;

- papier dominoté : impression à l'encre d'une planche de bois gravée ;

- papier marbré : dépose sur un bain d'eau couvert en surface d'encres grasses de couleur ;

- papier à vernis doré : impression au vernis d'une planche de bois gravée ;

- papier doré gaufré (ou papier brocart) : impression d'une plaque de cuivre gravée chauffée (motifs en creux ou en relief) sur un papier apprêté ;

- papier doré uni : impression d'une planche de cuivre unie sur un papier apprêté. 


\section{DÉCOR DES PLATS ET DOS}

On trouve sur les reliures courantes et soignées les mêmes types de fers que ceux utilisés sur les reliures de luxe. Ils peuvent être réunis en quatre catégories :

- fer plein : dessin entièrement doré (toutes périodes confondues);

- fer évidé : dessin dont le contour seul apparaît doré (mi xvI ${ }^{\mathrm{e}}$ siècle) ;

- fer azuré : dessin de fines hachures dorées (seconde moitié du XVI ${ }^{\mathrm{e}}$ siècle) ;

- fer filigrané : dessin de pointillé dense doré $\left(\mathrm{XvII}^{\mathrm{e}}\right.$ et tout début du XVIII ${ }^{\mathrm{e}}$ siècle).

\section{TYPOLOGIE DES RELIURES SOIGNÉES ET COURANTES}

Voir tableaux pp. 88-94. 


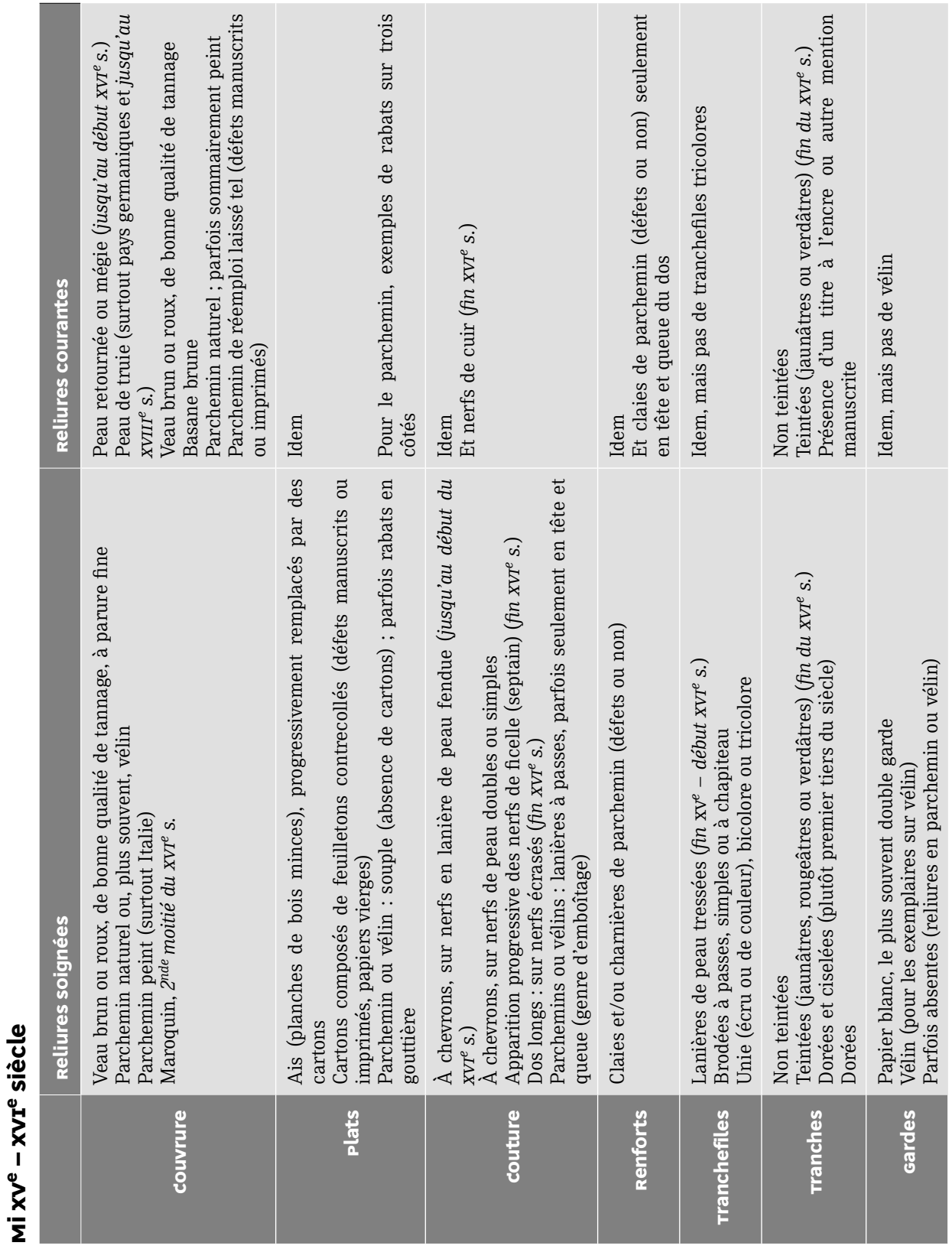




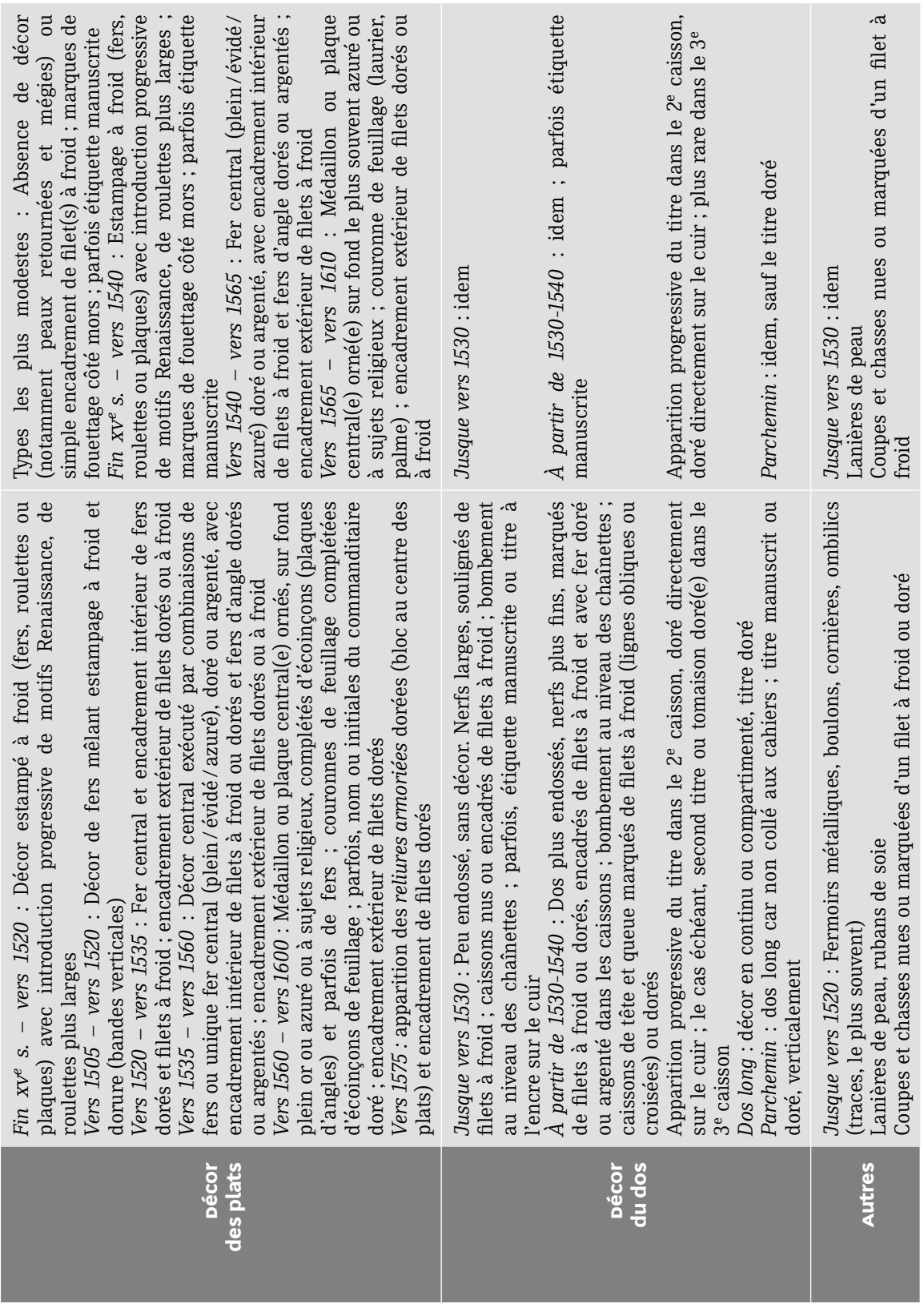




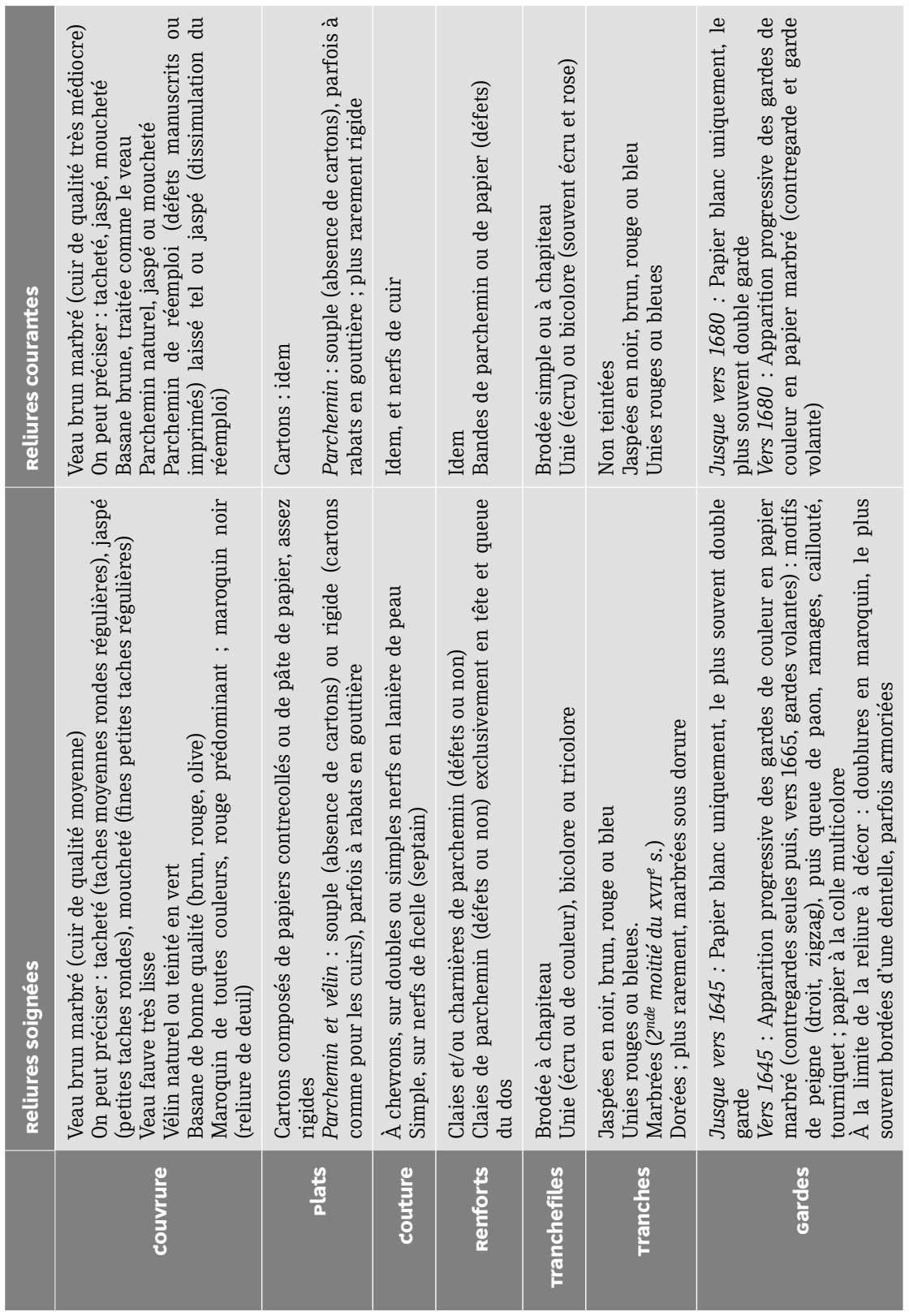




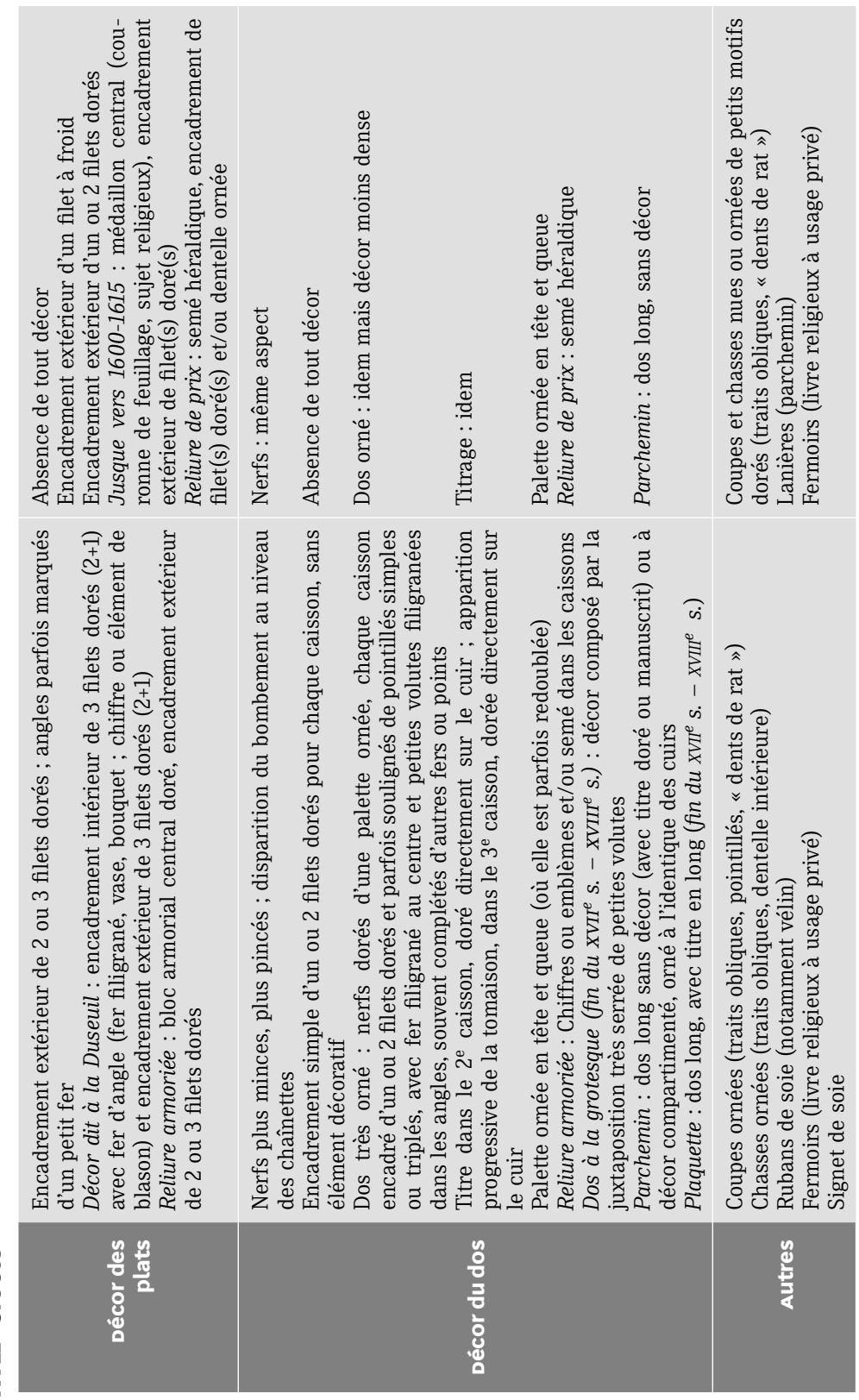




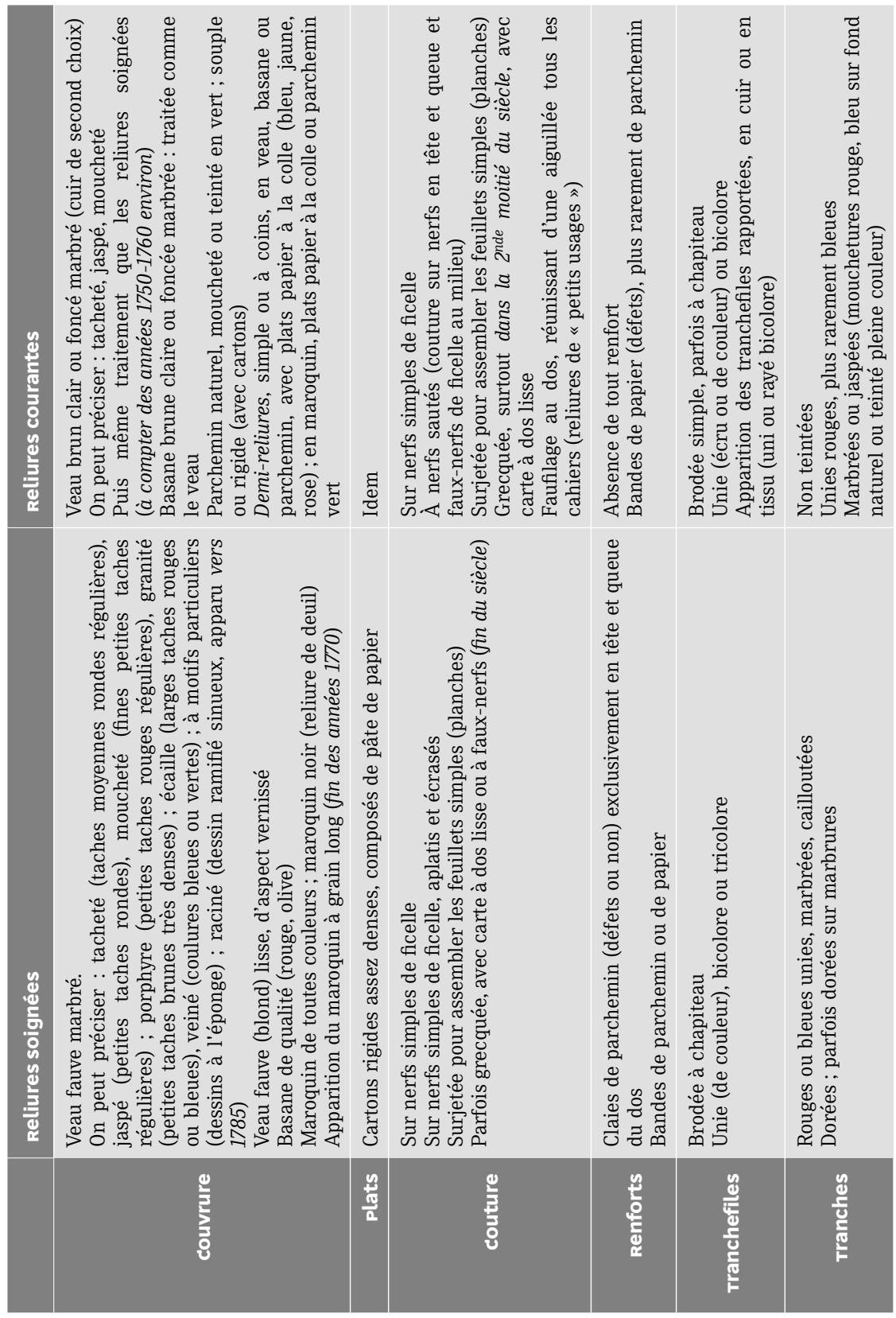




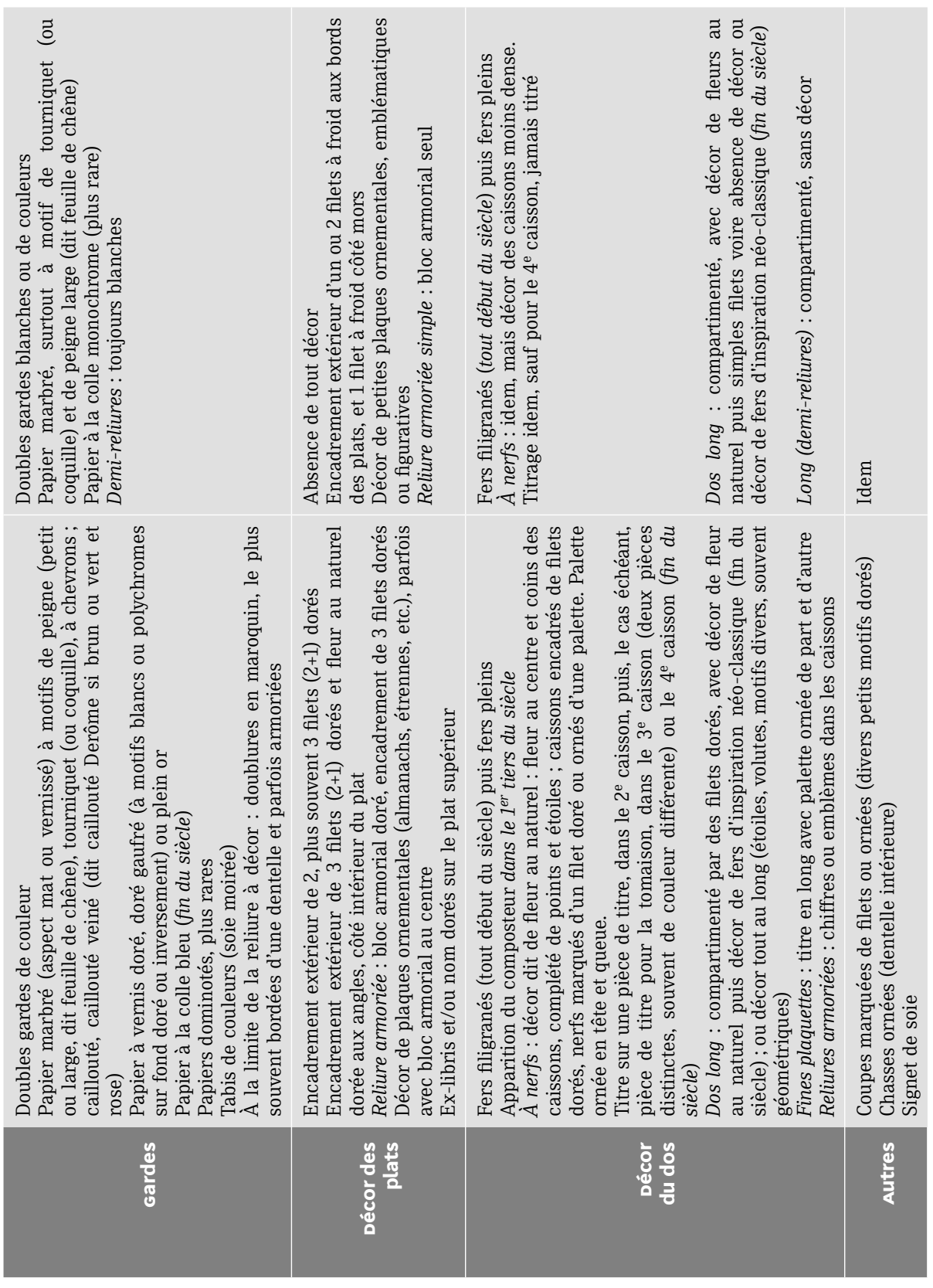




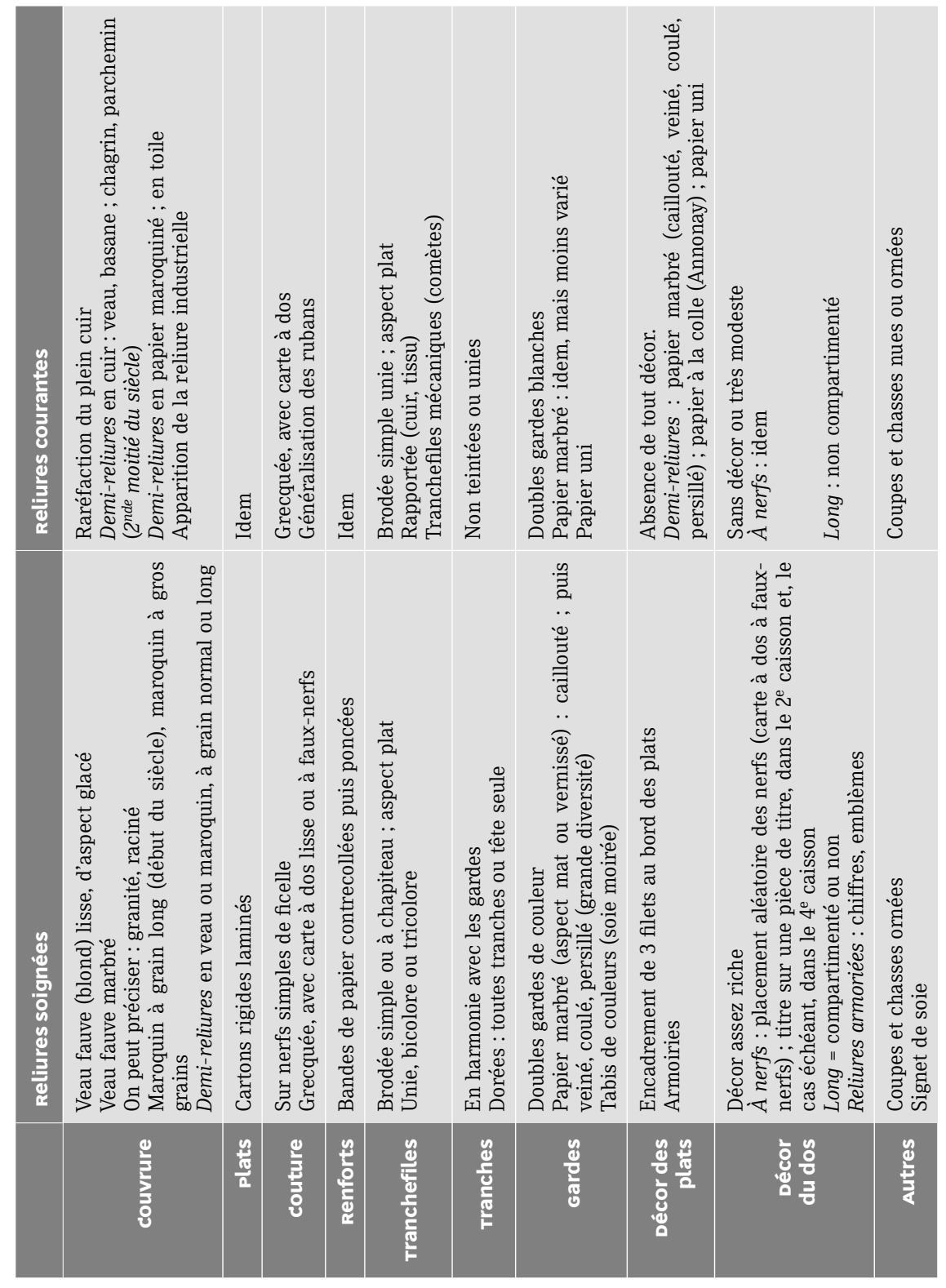




\section{3}

\section{IDENTIFIER LES TECHNIQUES ? 1}

Parmi les richesses et les difficultés que recèle l'étude de l'estampe, les techniques employées représentent un morceau de choix. Constitutives de l'histoire de cet art, leur reconnaissance est le préalable à toute entreprise de traitement d'un fonds d'estampes. Nous nous proposons dans ce chapitre de dresser un panorama des techniques en prenant soin de les restituer dans le contexte historique et de donner quelques éléments facilitant leur identification pour les non spécialistes.

Il n'est peut-être pas inutile, ici, de donner une définition de l'estampe. Une estampe est une image multipliable à l'identique faisant intervenir trois, voire quatre éléments : un élément d'impression ou matrice dont la nature varie en fonction de l'époque et du goût (bois, cuivre, pierre), de l'encre qui permet de transférer le dessin présent sur l'élément d'impression, un support pour recevoir l'impression de la matrice (papier, parchemin, tissu, etc.) et enfin, un moyen d'impression, généralement une presse adaptée à la nature de la matrice, mais se résumant parfois à une impression à la main.

Pour désigner une image obtenue par ce procédé, on peut employer le terme estampe ou gravure. Ces deux mots désignent deux réalités qui ne se recoupent pas entièrement. La gravure induit l'action d'entailler la matrice et comprend les techniques les plus anciennes du medium mais ce terme est impropre pour parler de la technique de la lithographie, procédé à plat intervenant très tard dans l'histoire de l'estampe, où l'artiste n'incise pas la pierre et ne fait que dessiner dessus. On l'aura donc compris, le terme estampe est plus large et sera privilégié quand on souhaite parler du medium de manière générale.

1. Pour mieux distinguer les différents procédés, il est conseillé de se reporter à la galerie d'images en ligne qui accompagne ce manuel : < http://www.enssib.fr/presses/galeriebao26 >. 


\section{LES TROIS GRANDES CATÉGORIES DE PROCÉDÉS}

Les techniques de l'estampe peuvent se répartir en trois grandes familles : les procédés en relief, les procédés en creux et les procédés à plat. Nous en donnons ci-contre un schéma synthétique.

\section{LES PROCÉdÉs EN RELIEF (TAILLE D'ÉPARGNE)}

Ils sont appelés ainsi car ce qui va donner le tracé du motif après impression est gravé en relief sur la matrice.

\section{Gravure en bois de fil ${ }^{2}$ et typographie}

La gravure en bois de fil consiste en l'utilisation d'une planche de bois débitée dans le sens du fil de l'arbre. Une fois le dessin réalisé sur la planche, le graveur va épargner le trait qui sera imprimé. Le principe de la gravure en bois repose sur le fait que seules les parties en relief, à fleur de la planche vont accepter l'encre, tandis que les parties qui doivent rester en blanc sur la feuille seront évidées. Pour faire en sorte qu'un trait soit imprimé, l'artiste exécute deux tailles parallèles à la ligne qu'il veut isoler et dégage donc tout autour le bois superflu. Il utilise différents instruments : la gouge dont la taille varie en fonction de la finesse de la ligne à graver, le canif pour les lignes plus épaisses et des ciseaux à bois pour évider les grandes surfaces de la planche.

Une fois la gravure achevée, la planche est encrée à l'aide d'un rouleau puis passée sous une presse. Puisque le tracé est en relief sur la planche, il n'est nul besoin d'une pression forte, mais verticale et uniforme. On peut même imprimer des planches de bois sans avoir recours à une presse. Il suffit d'appliquer le papier humidifié sur la planche, à l'aide d'un frotton une sorte de brosse dure ou baguette de bois lisse - ou tout autre objet arrondi, jusqu'à ce que l'encre se dépose sur le papier. Il s'agit sans doute de la technique d'impression des premières gravures en bois et également de la technique employée par les imprimeurs d'estampes japonaises traditionnelles.

2. On trouvera plus souvent dans la littérature l'expression « gravure sur bois » mais " gravure en bois » est le terme employé par les praticiens de ce medium dès le xvıII ${ }^{\mathrm{e}}$ siècle et nous le préférons. 

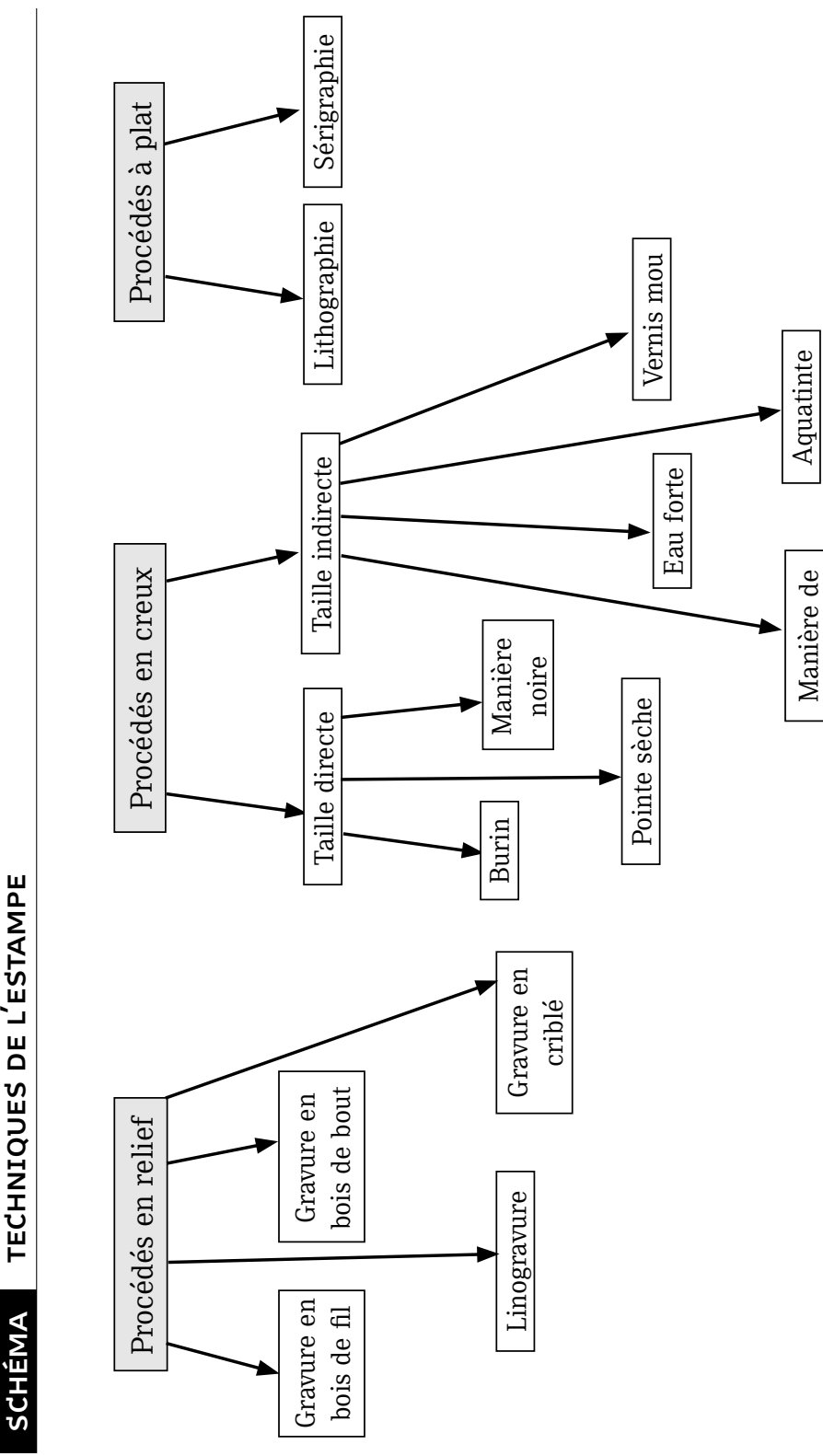
L'estampe gravée selon ce procédé ne possède pas de cuvette*. La gravure en bois de fil se reconnaît aux contrastes souvent marqués entre le trait et le blanc et au réseau de hachures qui bien souvent ne se recoupent pas. Cette technique est très économique, car elle permet de faire plusieurs dizaines de milliers de tirages.

La gravure en bois de fil est la première technique employée en Europe au début $d u x^{\mathrm{e}}$ siècle pour graver des sujets religieux et nourrir la dévotion contemporaine. Ces « incunables » de l'estampe sont donc antérieurs à l'invention des caractères mobiles d'imprimerie qui vont permettre l'impression de livres à partir des années 1450. La gravure en bois va trouver un nouvel essor grâce à cette révolution car imprimeurs et éditeurs de livres vont très vite comprendre l'intérêt de pouvoir combiner, sous la même presse, caractères d'impression et matrice gravée afin d'obtenir sur la même feuille texte et image imbriqués.

À partir des années 1930, un autre matériau est employé par les artistes de la même manière qu'une planche de bois de fil : le linoléum. Composé de poudre de liège et d'huile de lin, il est beaucoup plus tendre qu'une planche de bois. À l'impression, il est relativement difficile de faire la différence entre une planche de bois et un linoléum, sauf peut-être pour les grands aplats de noir, plus neigeux dans la gravure en bois.

\section{Bois de bout (ou debout)}

Cette technique consiste à couper le bois différemment de la gravure en bois de fil. Les planches de bois sont débitées en rondelles, perpendiculairement à l'axe du tronc. Dans la partie la plus dense de ces rondelles, on taille de petits cubes que l'on assemble à la colle et l'on forme ainsi une planche. On obtient ainsi un bois dur, particulièrement résistant, pris le plus souvent dans l'essence du buis. Le travail se fait également en relief, mais le graveur aura tendance à employer des instruments plus fins qui peuvent être d'ailleurs des instruments avec lesquels on travaille la gravure en métal, comme le burin.

Le procédé, développé par le graveur anglais Thomas Bewick (1753-1828), s'est diffusé au début du XIX ${ }^{\mathrm{e}}$ siècle en Angleterre pour l'illustration d'ouvrages très luxueux. À partir de 1830, il connut un grand succès, à la mesure du besoin d'illustration au $\mathrm{XIX}^{\mathrm{e}}$ siècle. Les compositions d'Honoré 
Daumier ou de Gustave Doré, qui les confiaient tous deux à des graveurs spécialisés, ont ainsi été exécutées dans cette technique, qui fut également employée pour l'illustration de journaux à grand tirage. Ce procédé, permettant des tirages à hauteur de plus de 100 000, était peu onéreux. Il fut ensuite progressivement abandonné dans la seconde moitié du $\mathrm{XIX}^{\mathrm{e}}$ siècle car concurrencé par les techniques de reproduction dites photomécaniques.

La gravure en bois de bout se reconnaît à une fine trame horizontale qui anime les arrière-plans des images. Elle est d'une grande finesse.

\section{criblé}

Bien qu'utilisant le métal, cette technique relève de la famille des procédés en relief. La technique du criblé consiste en la gravure d'une plaque de métal de la même manière qu'une planche de bois. Les traits sont détourés et les blancs creusés. Afin que le rendu ne soit pas trop contrasté et que les aplats que laisse le graveur ne soient pas d'un noir uni, le graveur crible sa plaque de petits trous plus ou moins rapprochés qui permettent de donner des demi-teintes. L'outil forme un petit cratère dont les bords ressortent sous la forme d'un bourrelet circulaire qui accroche l'encre, tandis que la partie creuse ne recevant pas d'encre donne le blanc à l'impression.

Le criblé est une technique employée pendant un temps assez limité, à partir de 1450 jusque dans les années 1550. On la trouve surtout dans l'illustration des livres d'heures imprimés parisiens de la fin du $x^{\mathrm{e}}$ siècle. Développée pour son rendu plus fin que la gravure en bois et correspondant sans doute aussi à un goût particulier, elle fut vite abandonnée au profit de la taille-douce qui permet un traitement plus subtil des différents plans de la composition.

\section{LES PROCÉdÉS EN CREUX (TAILle-doUCE)}

Cette famille de techniques est ainsi appelée car, contrairement aux procédés en relief, le graveur creuse la matrice par plusieurs entailles ou tailles. L'imprimeur ne dépose l'encre que dans ces incisions qui donnent le motif à l'impression. Elle se pratique essentiellement sur du métal (plaque de fer, cuivre ou zinc d'un ou deux millimètres d'épaisseur). 
Les procédés en creux nécessitent une presse à taille-douce d'une pression beaucoup plus importante que pour la gravure en bois, car il faut aller chercher l'encre logée dans les tailles de la plaque. L'encrage de la plaque et son essuyage sont des étapes capitales pour assurer un bon tirage. On place la plaque encrée sur le lit de la presse, et dessus, le papier humidifié ainsi que des langes destinés à amortir la pression très importante, visible au fait qu'après impression le papier est enfoncé là où se trouve la plaque et porte ainsi la trace d'une cuvette. La plaque et le papier passent en effet entre deux gros rouleaux. L'encre se dépose en relief sur le papier, à la différence des techniques en relief, où l'encre se dépose de manière plane. Le passage sous presse répété d'une plaque et son encrage ont tendance à fatiguer les tailles qui sont de moins en moins creuses et à donner un tirage de moins bonne qualité.

Ces étapes d'impression sont identiques à toutes les techniques relevant des procédés en creux. Ces dernières se différencient donc entre elles par la manière dont on grave la plaque.

\section{Les techniques de taille directe}

Elles sont ainsi appelées car la gravure se fait manuellement, directement à l'aide d'un outil.

\section{Burin}

Il s'agit d'un instrument doté d'une lame emmanchée dans une demi-poire de bois et dont la pointe se termine par un V. Le graveur place cette partie dans sa paume et maintient la lame entre son pouce et son index. C'est avec l'épaule, le bras et la paume que le graveur taille le métal et non avec les doigts. Pour réaliser des déliés et des lignes courbes, le graveur ne fait pas tourner son burin dont la pointe est toujours dirigée dans le même sens mais fait tourner la plaque. Les résidus de la plaque de métal résultant de l'incision de la plaque (appelés barbes) sont dégagés pour obtenir une taille nette. Les erreurs ou dérapages de l'instrument peuvent être réparés en utilisant un brunissoir pour aplanir la plaque ou en martelant la plaque au verso.

La pratique du burin demande une très grande habilité et une longue formation. C'est, après la gravure en bois de fil, la deuxième technique 
employée par les graveurs en Europe, à partir de 1430-1440 en Allemagne du sud. Elle connaît un âge d'or au Xvi ${ }^{\mathrm{e}}$ siècle avec des artistes comme Albrecht Dürer. Au XvII ${ }^{\mathrm{e}}$ siècle, en France, elle est portée à son excellence par de grands burinistes de formation comme Claude Mellan avec son chef d'œuvre, La Sainte Face ou Robert Nanteuil avec ses nombreux portraits gravés, en développant une syntaxe de tailles parallèles, croisées et de points extrêmement raffinée. Les effets d'ombre et de lumière résident en grande partie dans la profondeur des tailles et leur épaisseur.

Une gravure au burin se reconnaît à ses tailles qui commencent par une attaque franche et se continuent en s'élargissant. Contrairement à l'eauforte (voir page suivante), la taille gravée au burin est régulière sur tout son long.

\section{Pointe-sèche}

L'instrument appelé ainsi est une pointe d'acier emmanchée que l'on tient comme un crayon et qui égratigne la plaque plus ou moins profondément. À la différence du burin, le graveur prend soin de conserver les barbes du métal qui, une fois encrées, vont donner un trait velouté propre à cette technique. Tout l'intérêt de celle-ci consiste à garder le plus possible ces copeaux de métal qui auront tendance à s'atténuer et disparaître au fur et à mesure de l'encrage et du passage sous presse. La pointe-sèche ne permet donc pas de faire de nombreux tirages, une trentaine au grand maximum. Il s'agit d'une technique qui peut être employée seule, mais elle a souvent, dans l'histoire de l'estampe, été utilisée en complément d'une autre, le burin ou l'eau-forte. Elle est employée dès le xvı ${ }^{\mathrm{e}}$ siècle par Dürer, et Rembrandt en donnera des impressions saisissantes au XviI ${ }^{\mathrm{e}}$ siècle. Elle se reconnaît au noir baveux et souvent très chargé du trait.

\section{Manière noire}

Appelée aussi mezzotint en anglais ou ars nigra en latin, elle consiste en un travail des valeurs et non du trait, grâce à une préparation particulière de la plaque de cuivre. Avec un instrument appelé berceau constitué d'une lame épaisse striée verticalement d'un côté, au bord biseauté, légèrement arrondi et dentelé, promené sur toute la plaque, le graveur va entailler (bercer) de manière régulière le métal en constituant une fine trame ou 
une sorte de quadrillage. La plaque en surface apparaît comme grainée et hérissée d'une multitude de petites pointes. Si l'on devait encrer et imprimer la plaque à cette étape, on obtiendrait un noir complet, intense, du fait de ces pointes de la plaque qui auront accroché l'encre.

À partir de cette préparation, le graveur va, à l'aide de brunissoirs et de grattoirs, ôter ou écraser le grain pour obtenir des blancs ou des gris. Plus le grain sera écrasé, plus le graveur va vers le blanc. Il fait donc émerger des motifs du noir.

La manière noire a été inventée en 1642 par Ludwig von Siegen, un graveur allemand. Elle fut ensuite perfectionnée par des artistes français et néerlandais comme Wallerant Vaillant ou Abraham Blooteling. Elle connut un âge d'or en Angleterre au XvIII ${ }^{\mathrm{e}}$ siècle où elle servit à interpréter dans l'estampe les compositions de peintres comme Gainsborough.

La manière noire se reconnaît très facilement au fait qu'à l'examen attentif de l'estampe, on n'y décerne aucun trait. Les valeurs de noir et de gris sont veloutées. La trame laissée par le berceau est souvent encore visible au compte-fils.

\section{Les techniques dites de taille indirecte}

Elles sont ainsi désignées car elles font intervenir un agent chimique pour graver la plaque, de l'acide nitrique, appelée eau-forte. Aujourd'hui, on utilise surtout le perchlorure de fer.

\section{Eau-forte}

Le graveur recouvre l'ensemble de la plaque d'un vernis protecteur qui doit avoir bien séché. L'artiste grave son dessin sur ce vernis, avec un instrument très fin qui se manipule comme un crayon. Il peut s'agir d'une pointe ou d'une aiguille plus ou moins fine. Cet instrument raye le vernis et laisse le métal à nu. Une fois le dessin terminé, le graveur fait « mordre » la plaque dans l'acide, c'est-à-dire qu'il la laisse à tremper pendant quelques instants dans un bain d'acide. C'est l'action de l'acide qui, en se déposant sur les parties laissées nues du métal, va graver le dessin sur la plaque. Plus la zone est mordue par l'acide, plus la taille est profonde. 
Le vernis permet de stopper le travail sur une zone de la plaque que le graveur souhaite ne plus voir évoluer car le vernis protège la plaque de l'action de l'acide.

Plus encore que le burin, l'eau-forte permet à l'artiste de multiplier les états de son estampe, c'est-à-dire d'imprimer la plaque à un instant T de son travail. Les impressions intermédiaires lui donnent une bonne idée de l'avancée de la gravure et des zones à encore travailler.

L'eau-forte est plus accessible que le burin, car l'artiste réalise son dessin sur la plaque comme il le ferait sur un papier. L'instrument très simple laisse une grande liberté à l'artiste et c'est pour cette raison que l'eauforte est souvent appelée la technique des peintres-graveurs, plus que des graveurs spécialisés.

L'eau-forte fut expérimentée en Europe au début du XvI ${ }^{\mathrm{e}}$ siècle à Augsbourg et en Italie à partir des années 1530, puis en France en 1540. Elle reste très employée aux siècles suivants.

Par l'action de l'acide et le travail très libre de la pointe sur le vernis, la taille gravée par l'eau-forte ne ressemble pas à celle laissée par le burin, technique avec laquelle elle est parfois confondue. Son épaisseur peut varier de manière significative sur quelques millimètres, le trait se contorsionner, ressembler à un griffonnis. Il est d'autant plus important de pouvoir la différencier du burin que l'eau-forte a été souvent employée au XVII ${ }^{\mathrm{e}}$ et au XVIII ${ }^{\mathrm{e}}$ siècle avec le burin pour une même estampe.

\section{Vernis mou}

Il s'agit d'une manière particulière d'ôter le vernis de la plaque. On travaille la plaque avec un vernis qui n’a pas complètement séché. Il s'arrache de la plaque dès qu'on le soumet à la moindre pression. On appose ainsi une feuille de papier humide sur ce vernis mou, et l'on dessine son motif sur la feuille de papier avec un crayon à la mine dure. Lorsque l'on va ôter la feuille, les endroits où le dessin aura été tracé seront ceux où le vernis aura été enlevé de la plaque car resté collé sur la feuille de papier. La plaque est donc mise à nu de manière très nette. Elle est ensuite plongée dans de l'acide, de la même manière qu'une eau-forte. À l'impression, le rendu a un aspect crayonné. 
Le vernis mou a été développé par Giovanni Battista Castiglione en 1640 puis est devenu très en vogue au XvIII ${ }^{\mathrm{e}}$ siècle en Angleterre.

\section{Aquatinte}

Il s'agit d'une préparation spécifique de la plaque avant morsure par l'acide. On saupoudre la plaque d'une poudre de résine ou de colophane, que l'on fait chauffer pour qu'elle adhère parfaitement à la plaque, soit en utilisant une boîte à grain ou en déposant la poudre manuellement, à l'aide d'un tissu pour filtrer le grain. La plaque possède donc une trame constituée de cette poudre de résine qui forme des points irréguliers sur la surface de la plaque. Le graveur travaille sa plaque ensuite comme pour une eau-forte. Quand la plaque est plongée dans le bain, l'acide passe autour des grains, creuse le métal partout où il n'y a pas de résine. Ces légers creux retiendront l'encre à l'impression et donneront un rendu grainé, qui permettra d'avoir une trame de valeur intéressante.

L'aquatinte a été expérimentée par l'artiste français Jean-Baptiste Le Prince, dès 1768. Francisco Goya est l'un des artistes qui a porté cette technique à son apogée, en l'employant comme arrière-plan de ses eauxfortes. L'aquatinte se reconnaît à la trame laissée dans des aplats de valeur sur la gravure.

\section{Manières de crayon et de lavis}

La manière de crayon consiste en l'emploi d'une roulette striée de plusieurs pointes inégales que l'on fait rouler sur la surface de la plaque. L'instrument attaque le vernis en le piquetant, ce qui donne l'aspect d'un trait de crayon. Or, justement, la manière de crayon a été inventée par Demarteau (1722-1776) entre 1750 et 1755 pour donner à l'estampe la semblance d'un dessin. La plaque est ensuite mordue par l'acide. Quand l'estampe est encrée avec une encre de couleur bistre, la confusion avec un dessin à la sanguine est très facile ${ }^{3}$. Cette technique fut surtout employée dans la seconde moitié du XVIII ${ }^{\mathrm{e}}$ siècle.

La manière de lavis est contemporaine de la manière de crayon, mais perfectionnée pour donner le rendu d'un dessin lavé. Le graveur travaille

3. N'était la présence d'une cuvette dans le cas de l'estampe, à la différence du dessin. 
la plaque à l'eau-forte pour le dessin principal, puis ajoute au dessin des valeurs plus ou moins foncées par de larges aplats posés au pinceau trempé directement dans l'acide. Aux endroits très légèrement attaqués par l'acide déposé au pinceau, la plaque aura un grain plus ou moins fort qui retiendra l'encre à l'impression et donnera un léger ton. Elle a été développée en France par Jean-Charles François (1717-1769).

\section{LES PROCÉdÉS DITS À PLAT}

Ils sont appelés ainsi car ils ne font pas intervenir de gravure mais un transfert à plat d'un dessin. Nous ne parlerons pas de la sérigraphie, procédé récent en Occident et peu représenté dans nos bibliothèques. Nous nous contenterons d'évoquer ici la lithographie.

Ce procédé a été inventé par Aloys Senefelder (1772-1834), auteur de théâtre autrichien qui, cherchant à imprimer ses pièces de manière peu onéreuse, se mit à expérimenter l'impression sur pierre. La lithographie repose sur la propriété chimique de l'eau à repousser les corps gras. Un dessin tracé avec une substance grasse sur une pierre calcaire poreuse peut ainsi être imprimé en mouillant la pierre puis en l'encrant. L'eau ne reste que sur les surfaces non dessinées tandis que l'encre, hydrophobe car grasse, n'adhère qu'au dessin.

L'artiste dessine sur la pierre avec un crayon lithographique gras, à la plume ou au pinceau avec de l'encre lithographique. Il peut aussi utiliser des pointes, des grattoirs et des lames de rasoir pour ôter les excès de graisse.

Une autre possibilité de pratiquer la lithographie existe, à l'aide du papier report. Plutôt que de dessiner directement sur une pierre très lourde et peu pratique à emmener avec soi, artistes et graveurs vont développer le papier report sur lequel il est possible de dessiner directement à l'encre ou au crayon gras. Le papier est recouvert d'une couche de colle soluble à l'eau qui isole le crayon gras du papier. Ce dernier est ensuite transféré sur la pierre en le mouillant : la colle se dissout et la graisse de l'encre adhère à la pierre.

Une fois le dessin terminé, la pierre lithographique encrée avec la feuille de papier dessus est passée sous une presse dite lithographique, pourvue d'un râteau qui exerce une pression latérale et homogène. La lithographie 
a l'aspect d'un dessin. Il n'y a, sur le papier, aucune cuvette. L'imprimeur au XIX ${ }^{\mathrm{e}}$ siècle a souvent pris l'habitude, pour donner une teinte au papier, d'ajouter au moment de l'impression un papier de chine supplémentaire très fin de taille plus petite que la feuille de papier, qui par la pression, s'encastre parfaitement sur la feuille de papier.

La lithographie connut un succès très important en France où elle fut développée par l'atelier d'Engelmann à Paris, en 1816, et par Charles de Lasteyrie. Son âge d'or se poursuivit tout au long du XIX ${ }^{\mathrm{e}}$ siècle et elle fut employée par Delacroix, Géricault, ou encore Toulouse-Lautrec. Ce fut la technique utilisée pour les planches des Voyages pittoresques et romantiques dans l'ancienne France, publiés par Isidore Taylor et Charles Nodier entre 1820 et 1878 . Les affichistes de la seconde moitié du XIX ${ }^{\mathrm{e}}$ siècle l'utilisèrent presque exclusivement.

\section{ENCADRÉ DONNÉES À SIGNALER DANS UNE NOTICE D'ESTAMPE}

- le graveur : son nom est la plupart du temps gravé au bas de l'image, suivi de la mention latine sc. (ou sculp. pour sculpsit = " a gravé »), fecit (= " a fait »), inc. (pour incidit = « a gravé »).

Attention : la mention excud. ou excudit n'exprime pas le travail du graveur mais de l'éditeur de la gravure ;

- dans le cas de gravures réalisées à partir d'un dessin ou d'un tableau, le nom de l'artiste de l'œuvre représentée est indiqué également au-dessous de l'image, suivi de la mention inv. (pour invenit = " a trouvé ») ou pinx. (pour pinxit = " a peint ») ou delin. (pour delineavit = « a dessiné ») généralement à gauche ;

- le sujet ou le titre de l'estampe si celui-ci est gravé ;

- la date : comme elle est rarement expressément indiquée, il faut essayer de risquer une datation au siècle près ;

- les dimensions exprimées en mm ( $\mathrm{H} \mathrm{x} \mathrm{L)}$, sont celles de la cuvette (dans le cas d'une gravure en taille-douce) ou celles du trait cerné pour une gravure en bois ;

- la technique lorsqu'on a réussi à l'identifier. Sinon, se contenter d'indiquer les termes génériques : " gravure en bois », « taille-douce », « lithographie ». 


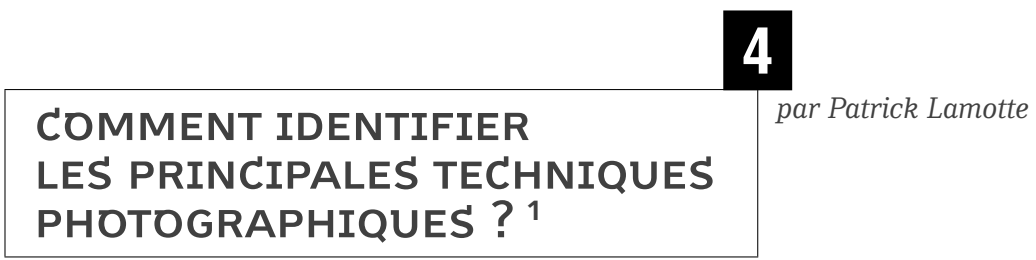

Quantité de photographies sont conservées dans les bibliothèques françaises depuis la première moitié du XIX ${ }^{\mathrm{e}}$ siècle. Le problème est que les bibliothécaires s'en sont rarement occupés, car ils étaient dans l'ignorance des diverses techniques photographiques et des modes de conservation que chacune nécessite ${ }^{2}$.

On peut classer les techniques photographiques en trois grandes familles :

- les positifs directs (image unique) ;

- les procédés négatifs (image unique);

- les procédés positifs (tirage multiple).

\section{TIRAGES POSITIFS PAPIER}

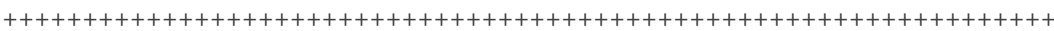

Les tirages positifs sur papier sont les plus fréquemment rencontrés dans les collections de bibliothèque :

- papier albuminé pour le XIX ${ }^{\mathrm{e}}$ siècle ;

- papier à développement pour le $\mathrm{xx}^{\mathrm{e}}$ siècle .

\section{PAPIER ALBUMINÉ}

$\mathrm{Au} \mathrm{XIX}^{\mathrm{e}}$ siècle, les premiers tirages positifs sur papier voient le jour entre 1840 et 1860 pour les papiers salés et 1850 à 1910 pour les papiers albuminés.

Le papier albuminé est né du fait que l'on s'est vite aperçu que dans le papier salé l'image allait se former en partie à l'intérieur des fibres du papier et donc perdait en contraste et en brillant.

1. Pour mieux distinguer les différents procédés, il est conseillé de se reporter à la galerie d’images en ligne qui accompagne ce manuel : < http://www.enssib.fr/presses/galeriebao26 >.

2. Comme l'a fait justement remarquer plus haut Laure Jestaz : " À quoi sert une réserve et qu'y mettre? », p. 40. 
Une épreuve sur papier albuminé est un tirage positif réalisé sur une feuille de papier enduite d'une couche d'albumine salée (blanc d'œuf contenant du chlorure de sodium) puis sensibilisée par flottaison dans une solution de nitrate d'argent.

L'image est obtenue par noircissement direct dans un châssis presse, puis virée et fixée.

La tonalité est chaude, de brun à noir bleuté. Les papiers albuminés ont un aspect satiné, mais ils peuvent présenter une surface brillante s'ils ont été doublement albuminés ou vernis.

L'albumine peut parfois être teintée en rose ou en bleu. Les images sont aux formats du négatif puis découpées et collées sur un support. Les épreuves se présentent généralement sur des cartons de montages car elles étaient très fines. Ces supports carton, souvent de médiocre qualité, deviennent cassants par leur acidité et mettent l'épreuve en danger.

Il en existe de nombreux formats :

- cabinet 10x14 cm image et 11x16,5 cm montage ;

- album 11x15 cm image et 12,5x16,5 cm montage ;

- vue stéréo 8,5x17,8 cm par exemple avec des variantes entre fournisseurs.

On constate le plus souvent un jaunissement de l'albumine.

Les tirages " carte de visite et cabinet » étaient souvent décorés d'un filet doré qui, en se dégradant, dépose de petites particules sur l'image. Il s'ensuit une oxydation locale de l'argent ; l'image est constellée de points clairs.

\section{PAPIER À DÉVELOPPEMENT}

$\mathrm{Au} \mathrm{xx}^{\mathrm{e}}$ siècle, les papiers sont généralement à développement, c'est-àdire un tirage photographique qui, par opposition au procédé à noircissement direct ou par contact, est développé, après exposition à la lumière pour que l'image apparaisse.

Ils furent fabriqués industriellement à partir de 1893. Le support est du papier baryté. Le sel sensible était du chlorure d'argent (papier gaslight), du chlorobromure ou du bromure d'argent dispersé dans la gélatine. 
Les surfaces sont mates, brillantes ou satinées, blanches ou légèrement colorées (crème chamois) d'aspect lisse, perlé ou grain de sable.

Présence fréquente dans les collections dans les formats usuels : 9x12, $13 \times 18,18 \times 24,24 \times 39,30 \times 40,40 \times 50$ et $50 \times 60 \mathrm{~cm}$.

La structure laminaire (papier/sulfate de baryum/couche image) rend ces procédés facilement distinguables des papiers albuminés et salés.

Après 1970 apparaissent les papiers photographiques plastifiés (papiers $\mathrm{RC}=$ Resin Coated). Il s'agit de papiers enduits de part et d'autre d'une couche de polyéthylène. L'émulsion photographique est ajoutée par-dessus l'une des deux couches.

\section{Les procédés photographiques couleur du $\mathrm{xx}^{\mathrm{e}}$ siècle}

\section{Développement chromogène (de 1942 à nos jours)}

C'est la société Agfa, en 1939, qui a introduit en premier sur le marché un film couleur négatif et le papier permettant de produire les tirages positifs. La firme KODAK suit en 1942 avec les Kodacolor mais le prix de traitement retarde le développement auprès du grand public. En France, c'est seulement dans les années 1970 que la photographie en couleur explose et remplace la photographie en noir et blanc. En 1990, elle représente $90 \%$ du marché et en 2005 elle atteint 97,5\%.

Le support est recouvert de trois couches d'émulsion au gélatinobromure d'argent, sensibilisées à un des domaines spectraux de la sélection trichrome : la première est sensibilisée aux rouges, la seconde aux verts, la troisième aux bleus.

Dans les couches sont introduits des coupleurs. Ce sont des composés chimiques incolores qui réagiront pendant le développement pour générer les colorants.

Après exposition sous un négatif couleur, l'émulsion est développée dans un révélateur chromogène. Aux endroits où l'argent métallique s'est formé, le révélateur se combine avec les coupleurs pour produire un colorant azométhinique de la couleur complémentaire au domaine de sensibilisation : la couche sensibilisée aux rouges génère un colorant cyan, celle 
sensibilisée aux verts un colorant magenta, et celle sensibilisée aux bleus un colorant jaune.

On obtient alors une image positive présentant toutes les couleurs complémentaires du négatif constitué de colorants organiques dans une couche de gélatine. Un bain de blanchiment puis de fixage permet de supprimer tous les composés argentiques résiduels.

La vitesse d'altération varie selon les marques et les générations de papiers. À partir des années 1980, ils sont beaucoup plus stables. Leur stabilité est estimée à une centaine d'années (Fuji cristal archive, Kodak Endura...).

Cependant, les colorants organiques se dégradent à la lumière et dans l'obscurité, ce qui peut entraîner une coloration rougeâtre ou bleuâtre si l'une des trois couches s'altère.

Le seul moyen de retarder la décoloration des tirages est de les conserver à basse température et de les exposer le moins possible à la lumière.

\section{LES PROCÉDÉS NÉGATIFS}

$++++++++++++++++++++++++++++++++++++++++++++++++++++++++++++++++$ ENCADRÉ 1

IDENTIFICATION ET SUPPORT DES NÉGATIFS MONOCHROMES

Support en papier

- Support mat

- Calotype (1841-1860)

- Support translucide

- Papier ciré

- Calotype ciré

- Négatif Eastman (petit format)

Support en verre

- Image tons crème

- Plaque à l'albumine (1848-1860)

- Collodion humide (1851-1885)

- Collodion sec (1855-1885)

- Image tons neutres

- Négatif au gélatino-bromure d'argent (1880-1940)
Support souple transparent

(1889 à nos jours)

- Négatif au gélatino-bromure d'argent sur support en :

- Nitrate de cellulose

- Diacétate de cellulose

- Triacétate de cellulose

- Polyester 
Le négatif le plus fréquemment rencontré dans les collections est le négatif sur verre au gélatino-bromure d'argent.

\section{NÉGATIF SUR VERRE AU GÉLATINO-BROMURE D'ARGENT (1880-1940)}

Présenté pour la première fois à l'Académie des sciences par Richard Lead Maddox, le procédé au gélatino-bromure d'argent remplacera progressivement tous les autres procédés photographiques, la gélatine devenant à la fin du $\mathrm{XIX}^{\mathrm{e}}$ siècle le médium indispensable pour la préparation des surfaces sensibles. Les plaques au gélatino-bromure d'argent alliant en effet une sensibilité accrue à une simplicité d’utilisation inégalée.

Alors qu'une plaque de verre au collodion humide doit être exposée et traitée dans les minutes qui suivent sa préparation, la plaque de verre au gélatino-bromure d'argent peut se conserver longtemps avant d'être utilisée.

Réalisation :

- une plaque de verre est enduite d'une solution chaude de gélatine mélangée à du bromure de cadmium et à du nitrate d'argent ;

- une fois séchée, la plaque photosensible est soumise à une phase de maturation qui consiste à conserver l'émulsion plusieurs jours à $32^{\circ} \mathrm{C}$. On augmente ainsi substantiellement la sensibilité de cette dernière, les temps d'exposition de plusieurs secondes étant raccourcis à quelques fractions de seconde seulement ;

- après exposition, la plaque est développée, fixée et lavée selon les méthodes classiques.

Précaution :

- ranger dans des pochettes ;

- ranger verticalement sur le grand chant*, par petit nombre, dans des boîtes au format. 
Le gélatino-bromure d'argent sera véritablement le premier procédé photographique industriel. Dès le début du $\mathrm{xx}^{\mathrm{e}}$ siècle, de nombreux établissements le commercialisent ; parmi eux, on citera notamment :

- Lumière (France) ;

- Agfa (Allemagne) ;

- Eastman [Kodak] (USA) ;

- Ilford (GB).

Les tonalités de l'image sont gris neutre, les surfaces sont mates, brillantes, ou satinées, blanches ou légèrement coloriées (crème, chamois), d'aspect lisse, perlé ou grain de sable.

$\mathrm{Au}$ début $\mathrm{du} \mathrm{xx}^{\mathrm{e}}$ siècle, la production journalière des usines Lumière est de 70000 plaques photographiques.

\section{IDENTIFICATION DES DIFFÉRENTS TYPES}

\section{TABLEAU DE NÉGATIFS SUR VERRE}

\begin{tabular}{|c|c|c|c|}
\hline & $\begin{array}{l}\text { Négatif à } \\
\text { l'albumine }\end{array}$ & $\begin{array}{l}\text { Négatif au } \\
\text { collodion }\end{array}$ & $\begin{array}{l}\text { Négatif au gélatino- } \\
\text { bromure d'argent }\end{array}$ \\
\hline $\begin{array}{r}\text { Période } \\
\text { chronologique }\end{array}$ & 1848 à 1860 & 1851 à 1885 & 1880 à 1940 \\
\hline \multirow[t]{6}{*}{$\begin{array}{r}\text { caractéristiques } \\
\text { physiques }\end{array}$} & $\begin{array}{l}\text { tonalité ocre- } \\
\text { orangé à vert olive }\end{array}$ & $\begin{array}{l}\text { tonalités chaudes: } \\
\text { crème, beige clair } \\
\text { à brun sombre }\end{array}$ & $\begin{array}{l}\text { tonalité neutre, } \\
\text { grise à noire }\end{array}$ \\
\hline & couchage irrégulier & couchage irrégulier & couchage uniforme \\
\hline & $\begin{array}{l}\text { formats atypiques } \\
\text { fréquents }\end{array}$ & $\begin{array}{l}\text { grands formats et } \\
\text { formats atypiques } \\
\text { fréquents }\end{array}$ & $\begin{array}{l}\text { formats } \\
\text { standardisés }\end{array}$ \\
\hline & $\begin{array}{l}\text { verres épais, } \\
\text { parfois mal coupés }\end{array}$ & $\begin{array}{l}\text { verres épais, } \\
\text { parfois mal coupés }\end{array}$ & $\begin{array}{l}\text { verres fins (moins } \\
\text { de } 2 \mathrm{~mm} \text {. }\end{array}$ \\
\hline & & $\begin{array}{l}\text { absence d'image } \\
\text { dans les angles }\end{array}$ & \\
\hline & $\begin{array}{l}\text { sur fond noir, } \\
\text { l'image apparaît } \\
\text { en positif }\end{array}$ & $\begin{array}{l}\text { sur fond noir, } \\
\text { l'image apparaît } \\
\text { en positif }\end{array}$ & \\
\hline \multirow[t]{2}{*}{$\begin{array}{l}\text { Altérations } \\
\text { spécifiques }\end{array}$} & & \multirow{2}{*}{$\begin{array}{l}\text { réseau de } \\
\text { craquelures à } \\
\text { partir des bords }\end{array}$} & $\begin{array}{l}\text { décollement de la } \\
\text { couche de gélatine }\end{array}$ \\
\hline & & & miroir d'argent \\
\hline
\end{tabular}




\section{NÉGATIF SOUPLES AU GÉLATINO-BROMURE D'ARGENT (1889 À NOS JOURS)}

À partir de 1889, des matières synthétiques comme le nitrate de cellulose sont employées par Kodak comme supports en photographie et en cinématographie jusqu'en 1951, date à laquelle ils sont interdits car jugés trop inflammables (film auto-inflammable et auto-combustible).

\section{AUTRES SUPPORTS}

D’autres supports vont se développer grâce au développement des industries chimiques des plastiques entre les deux guerres. Les principaux matériaux utilisés sont les suivants :

- diacétate de cellulose (1923) ;

- acétopropionate de cellulose (1930, films cinéma) ;

- acétoburate de cellulose (1930, plans-films et films radiographiques) ;

- chlorure de polyvinyle (1945, films pour les arts graphiques);

- triacétate de cellulose (1948) ;

- polystyrène (1954) ;

- polyester (1955).

Les supports souples que l'on retrouve le plus souvent dans les bibliothèques sont le nitrate de cellulose, le diacétate de cellulose, le triacétate de cellulose et le polyester.

Aujourd'hui, le triacétate de cellulose et le polyester sont encore employés pour la fabrication des supports souples.

Les formats sont standardisés : 6x9, 9x12, 13x18, 18x24, etc. 


\section{ENCADRÉ 2 PRINCIPALES ALTÉRATIONS DU SUPPORT}

Le nitrate de cellulose est la « bête noire » des conservateurs. Il faut les identifier car ils ont un fort risque d'altération :

- stade 1 : coloration de jaune à jaunebrun, voire brun foncé. Un miroir d'argent se forme ;

- stade 2 : en milieu humide, l'émulsion devient poisseuse et colle. En milieu sec, le support devient cassant ;

- stade 3 : le support devient très fragile et dégage de l'acide nitrique (odeur forte) et du dioxyde d'azote. L'ensemble de la collection est menacé : attaque du support lui-même, des objets environnants et risque d'incendie ;
- stade 4 : les négatifs sont collés entre eux et avec leurs enveloppes : images perdues et négatifs à détruire pour qu'ils n'altèrent plus le reste de la collection ;

- stade 5 : dépôt d'une poudre brunâtre.

Considéré comme produit dangereux du fait de son inflammabilité et des risques d'explosion qu'il induit, le nitrate de cellulose est l'objet d'une législation stricte (loi n 76-663 du 19 juillet 1976). Au-delà de 50 kg par lieu de stockage (c'est-à-dire par bâtiment ne répondant pas aux normes de stockage de produits toxiques, quelle que soit sa taille), l'institution ou la personne qui en détient est dans l'obligation d'effectuer une déclaration auprès de la Préfecture. 


\section{TABLEAU PRINCIPAUX PROCÉDÉS PHOTOGRAPHIQUES}

\begin{tabular}{|c|c|c|}
\hline Procédés positifs directs & Procédés positifs & Procédés négatifs \\
\hline \multirow[t]{3}{*}{$\begin{array}{l}\text { Daguerréotype } \\
1839-1860\end{array}$} & $\begin{array}{l}\text { Dessin photogénique } \\
1834-1840\end{array}$ & \\
\hline & $\begin{array}{l}\text { Papier salé } \\
1840-1860\end{array}$ & \\
\hline & $\begin{array}{l}\text { Cyanotype } \\
1842-1860\end{array}$ & $\begin{array}{l}\text { Calotype (négatif papier) } \\
1841-1860\end{array}$ \\
\hline $\begin{array}{l}\text { Pannotype } \\
1853-1880\end{array}$ & $\begin{array}{l}\text { Papier albuminé } \\
1850-1910\end{array}$ & $\begin{array}{l}\text { Procédé à l'albumine } \\
1848-1860\end{array}$ \\
\hline $\begin{array}{l}\text { Ambrotype } \\
1854-1870\end{array}$ & & $\begin{array}{l}\text { Procédé au collodion } \\
\text { humide ou au collodion sec } \\
\text { 1851-1885 }\end{array}$ \\
\hline \multirow[t]{2}{*}{$\begin{array}{l}\text { Ferrotype } \\
1853-1930\end{array}$} & $\begin{array}{l}\text { Platinotype } \\
1873-1930\end{array}$ & \\
\hline & & $\begin{array}{l}\text { Procédé au gélatino- } \\
\text { bromure d'argent } \\
\text { 1880-1940 }\end{array}$ \\
\hline $\begin{array}{l}\text { Vues de lanterne magique } \\
\text { et vues stéréoscopiques } \\
1850-1950 \\
\text { Papier salé } \\
1840-1860\end{array}$ & $\begin{array}{l}\text { Aristotype } \\
\text { (papier baryté) } \\
\text { à la gélatine ou } \\
\text { au collodion } \\
1860-1940\end{array}$ & $\begin{array}{l}\text { Procédé au gélatino- } \\
\text { bromure d'argent sur } \\
\text { support souple } \\
1889 \text { à nos jours }\end{array}$ \\
\hline $\begin{array}{l}\text { Diapositives } \\
\text { trichromes Lumière } \\
1893-1900\end{array}$ & $\begin{array}{l}\text { Papier à } \\
\text { développement } \\
1893 \text { à nos jours }\end{array}$ & \\
\hline \multicolumn{3}{|l|}{$\begin{array}{l}\text { Autochrome } \\
1907-1935\end{array}$} \\
\hline \multirow[t]{2}{*}{$\begin{array}{l}\text { Kodachrome } \\
1935\end{array}$} & & \\
\hline & $\begin{array}{l}\text { Papiers couleurs } \\
\text { à développement } \\
\text { chromogène } \\
1942\end{array}$ & $\begin{array}{l}\text { Négatifs couleurs à } \\
\text { développement chromogène } \\
1939\end{array}$ \\
\hline
\end{tabular}




\section{SCHÉMA}

REPRÉSENTATION DES ÉPREUVES PHOTOGRAPHIQUES SUR PAPIER

Papier salé, cyanotype, platinotype :

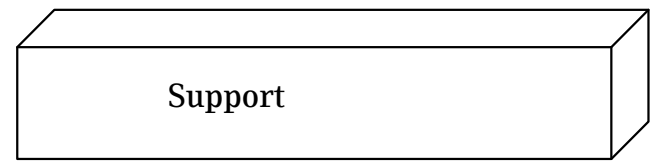

Épreuves à l'albumine, woodburytype, épreuve au charbon :

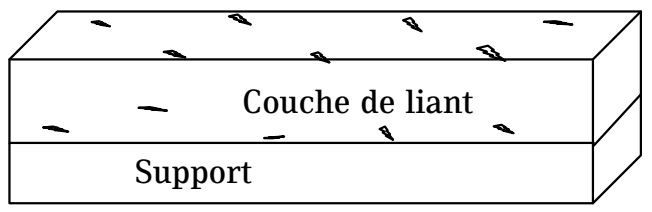

Papiers à émulsion, à noircissement direct, par développement :

\begin{tabular}{|l}
\hline Coüche de liant - \\
\hline Couche barytée \\
\hline Support \\
\hline
\end{tabular}




\section{5}

DÉCRIRE ET SIGNALER DES DOCUMENTS PATRIMONIAUX : Florent Palluault ENJEUX, FORMATS, PERSPECTIVES

Les catalogues permettent au bibliothécaire chargé des collections patrimoniales de passer d'une appréciation floue à une gestion efficace des collections et à une valorisation pertinente des documents. Cependant, tout professionnel est tributaire de l'état du signalement dont il hérite à son entrée en fonction. Or les évolutions de l'informatique documentaire ont prioritairement bénéficié au catalogage des nouvelles acquisitions. Trop nombreux sont encore les fonds patrimoniaux dont le signalement est insatisfaisant, incomplet, ou même inexistant.

L'enquête sur les fonds patrimoniaux des bibliothèques territoriales lancée en 2004 par la Direction du livre et de la lecture du ministère de la Culture et de la Communication dans le cadre du Plan d'action pour le patrimoine écrit*1 a montré qu'un tiers des collections, tous supports confondus, n'avait fait l'objet d'aucun traitement initial, et qu'un peu moins de la moitié n'était pas cataloguée. En 2007, 40\% seulement des collections patrimoniales étaient décrites dans des catalogues informatisés, cette proportion étant plus importante pour les livres imprimés que pour les autres types de document.

Les utilisateurs sont habitués aujourd'hui à " tout " trouver immédiatement en ligne, et ils acceptent mal que des catalogues ne soient pas informatisés ou soient incomplets. Combler les lacunes du signalement informatisé des fonds patrimoniaux des bibliothèques demeure donc un objectif prioritaire, aussi bien pour communiquer en salle de lecture, pour offrir une visibilité internationale au patrimoine français, que pour satisfaire le besoin en métadonnées induit par les programmes de numérisation de documents anciens et spécialisés.

1. Gérard Cohen, Michel Yvon, "Le plan d'action pour le patrimoine écrit », Bulletin des bibliothèques de France, 2004, t. 49, n 5, pp. 48-50. [En ligne] : < http://bbf.enssib.fr/consulter/bbf2004-05-0048-008 >. 


\section{QUELLES SONT LES OPTIONS DE SIGNALEMENT ?}

$++++++++++++++++++++++++++++++++++++++++++++++++++++++++++++++++++$

\section{SIGNALER LES FONDS}

Pour signaler des fonds non-inventoriés, par exemple à réception d'un don ou legs, des solutions simples peuvent être mises en œuvre :

- description courte sur le site Web de la bibliothèque ;

- description rapide (identification, origine, volumétrie, thématique) au format EAD (Description archivistique encodée) ;

- description dans le Répertoire national des bibliothèques et des fonds documentaires (RNBFD) du Catalogue collectif de France $(\mathrm{CCFr})^{2}$.

\section{CATALOGUER LES DOCUMENTS}

Pour fournir aux usagers un signalement plus précis, une approche document par document est nécessaire. Le catalogage rétrospectif - ou le recatalogage (lorsque les inventaires existants sont trop partiels ou sommaires) - fournit des notices de qualité, mais se révèle très coûteux en temps. Si les masses documentaires sont trop importantes pour les moyens du service, il est préférable de passer un marché de catalogage auprès d'un prestataire.

\section{INFORMATISER LES ANCIENS CATALOGUES}

La conversion rétrospective des catalogues et inventaires (ou " rétroconversion ») se base sur les données présentes dans un catalogue papier (registres, fiches) ou dans un catalogue informatique structuré dans un format autre que les formats documentaires habituels (traitement de texte, tableur, base de données générique, etc.). Les notices bibliographiques rédigées avant l'émergence de la normalisation documentaire sont souvent très lacunaires. Une numérisation suivie de reconnaissance des caractères (océrisation) ou de ressaisie des données peut donc se révéler

2. Catalogue collectif de France : < http://ccfr.bnf.fr >. Le RNBFD recense environ 2000 fonds patrimoniaux, locaux et spécialisés. Ces fiches de fonds complètent utilement les 5000 notices d'établissements du RNBFD. Des programmes systématiques d'enrichissement, via les pôles associés régionaux de la $\mathrm{BnF}$, sont appelés à se développer. Ces notices de fonds sont structurées dans un format proche de l'EAD. 
insatisfaisante. Pour les documents en multiples exemplaires (ouvrages imprimés, estampes), on privilégie l'identification dans les réservoirs nationaux pour dériver des notices fiables et complètes ${ }^{3}$. Le recours à un prestataire extérieur permet une production rapide des notices, l'équipe de la bibliothèque se chargeant du contrôle de la qualité du travail réalisé et de l'enrichissement éventuel des notices (données d'exemplaires, indexation). Quel que soit le format cible, un cahier des charges précis permettra de se prémunir contre des résultats décevants. Il devra préciser le format souhaité et donner les règles de création des notices en entrant dans le détail des zones ou éléments voulus, le codage des caractères, etc.

\section{Soutien financier aux opérations d'informatisation}

Les bibliothèques qui envisagent des projets d'informatisation ${ }^{4}$ de catalogues peuvent bénéficier de l'expertise scientifique et d'un éventuel soutien financier de la part des acteurs nationaux et régionaux du signalement : ministère de la Culture et de la Communication ${ }^{5}$, BnF, structures régionales du livre, Agence bibliographique de l'enseignement supérieur $(\mathrm{ABES})^{6}$.

\section{NORMALISATION DOCUMENTAIRE ET FORMATS INFORMATIQUES}

$++++++++++++++++++++++++++++++++++++++++++++++++++++++++++++++++++$

Les collections patrimoniales des bibliothèques correspondent à l'ensemble des documents conservés dans une perspective à long terme ${ }^{7}$. Cette définition recouvre donc tous types de document, manuscrits, documents d'archives et imprimés anciens, mais également certains périodiques et livres contemporains (livres d'artistes, par exemple), cartes et plans, partitions musicales, monnaies et médailles, objets, enregistrements sonores, images fixes, images animées, ressources multimédia et

3. Cf. Fiche Récupération de données pour le catalogue $:<$ http://www.patrimoineecrit.culture. gouv.fr/Cata/BAON_Fiche1_Recuperation_donnees.pdf $>$.

4. Voir Thierry Claerr et Isabelle Westeel, Numériser et mettre en ligne, Villeurbanne, Presses de l'enssib, 2010.

5. Voir le site Patrimoine écrit $:<$ http://www.patrimoineecrit.culture.gouv.fr/PAPE.html >.

6. Voir l'article de Thierry Claerr : "Inscrire son projet dans les politiques patrimoniales nationales et régionales », p. 152.

7. Voir plus haut l'article de Bernard Huchet : «Patrimoine et politique documentaire : "la bibliothèque dans son entier" ", pp. 33-34. 
même ressources électroniques. Les enjeux spécifiques au signalement des documents patrimoniaux ne concernent donc pas tant la typologie documentaire que la complexité et l'« épaisseur historique » inhérente à ces documents. Unicité, rareté, difficulté d'identification, contexte de production, particularités physiques, histoire, anciens possesseurs, reliure ou conditionnement constituent autant d'informations, parfois malaisées à découvrir, qui font la richesse d'une notice descriptive.

L'informatisation de ces notices pose à la fois la question de la collecte des données descriptives et celle de leur structuration : elle exige de connaître les formats recommandés pour les divers types de document. Depuis les décennies 1970-1980, l'effort de normalisation documentaire a entraîné l'utilisation des règles internationales de description bibliographique ISBD et de leurs transcriptions françaises, puis de l'ISBD intégré (2007) ${ }^{8}$. Cela s'est traduit par la généralisation de catalogues informatisés structurés au format MARC. À partir des années 2000, toutefois, de nouveaux formats basés sur le langage XML, et exprimés par des DTD ou des schémas XML, ont commencé à être utilisés à divers degrés dans les bibliothèques.

\footnotetext{
Le XML

Langage de balisage étendu, qui permet d'encoder et de structurer des informations. Indépendant des plates-formes informatiques, des systèmes d'exploitation ou de logiciels particuliers, et donc propice à l'échange de données, le XML a l'avantage d'utiliser la très large palette d'écritures, de caractères et de symboles de l'Unicode.

Une Définition de type de document (DTD) est à la fois un vocabulaire, qui définit les éléments et attributs autorisés en précisant leur caractère obligatoire ou facultatif, répétable ou unique, et une grammaire, qui indique à quels endroits et dans quel ordre utiliser ces éléments et attributs. Les schémas XML sont une forme plus aboutie de DTD et permettent un contrôle des données saisies et peuvent être associés à d'autres schémas.
}

8. Les normes donnant les règles de rédaction des descriptions bibliographiques ( $\mathrm{Z}$ 44-074 [livre ancien], Z 44-066 [enregistrements sonores], Z 44-065 [images animées], Z 44-077 [image fixe], Z 44-082 [ressources électroniques], Z 44-067 [ressources cartographiques], Z 44-069 [musique imprimée]) et celles donnant les règles de rédaction des accès ( $Z$ 44-059 [choix des accès], Z 44-060 [collectivités], Z 44-061 [personnes physiques, titres uniformes textuels, titres de forme], Z 44-079 [titres uniformes musicaux], Z 44-081 [vedettes cartographiques]) vont être révisées suite à l'adoption de l'ISBD intégré. 


\title{
LE FORMAT MARC
}

Fondé sur les règles ISBD, le MARC structure l'information bibliographique en la codifiant en zones et sous-zones, et éventuellement en positions au sein de ces sous-zones, pour être interprétée par une machine et faciliter les échanges. Deux formats MARC sont utilisés en France : l'Intermarc à la BnF et l'Unimarc dans les autres établissements documentaires. Même s'ils ont été conçus au départ pour les livres imprimés, les formats MARC ont l'ambition de décrire tous les types de document couverts par les ISBD. Ainsi, en Unimarc, certaines zones codées sont destinées à accueillir des informations spécifiques à certains types de ressources, par exemple les zones 115 (documents audiovisuels), 116 (documents graphiques), 117 (objets), 120-121, 123-124 et 131 (documents cartographiques), 125 à 128 (ressources musicales et sonores) ${ }^{9}$. D'autres zones MARC sont particulièrement utiles pour la description des documents patrimoniaux : zones de notes consacrées à la collation (307), l'exemplaire (316), la provenance (317), ou bien aux documents reliés avec celui qui fait l'objet de la notice (481).

\begin{abstract}
Livre ancien
Les zones codées spécifiques permettent d'entrer assez finement dans la description des particularités du document. Pour la description du livre ancien, par exemple, on peut donner en $140 \$$ a des informations sur les illustrations dans le texte (positions 0-3) ou hors texte (pos. 4-7), la technique d'illustration (pos. 8), le support (pos. 20), le filigrane (pos. 22), la marque d'imprimeur (pos. 23) ou d'éditeur (pos. 24). On peut également donner en 141 des données sur la reliure de l'exemplaire décrit (matériau, description, état matériel).

Des précisions sur les modalités de catalogage du livre ancien seront bientôt apportées par la révision de la norme de catalogage du livre ancien (Z44-074), dont le champ d'application est le livre imprimé durant la période 1501-1830. Une enquête de 2006 a montré que cette norme était fréquemment utilisée en bibliothèque mais qu'il était nécessaire de la refondre et de fournir aux catalogueurs un manuel d'application aux formats MARC.
\end{abstract}

9. Fédération internationale des associations de bibliothécaires et de bibliothèques, Universal bibliographic control and international MARC programme, Manuel UNIMARC : format bibliographique ; traduit par le Comité français UNIMARC, 5e éd. Munich, K.G. Saur, 2007. 
Les opérations de conversion rétrospective de notices de monographies imprimées ont créé, depuis le début des années 1990, une immense masse de données bibliographiques en MARC ${ }^{10}$ :

- les données des bibliothèques territoriales et des établissements relevant de l'Enseignement supérieur, en Unimarc, ont respectivement alimenté la Base Patrimoine du CCFr et le Système universitaire de documentation (Sudoc) ;

- les collections de la BnF ont été converties en Intermarc.

D'autres types de document patrimonial ont bien évidemment été décrits en MARC :

- les périodiques imprimés, disponibles dans le Sudoc, héritier du catalogue collectif national des publications en série ;

- des sources musicales manuscrites et imprimées du Xvi ${ }^{\mathrm{e}}$ au $\mathrm{xx}^{\mathrm{e}}$ siècle, issues de l'informatisation de Patrimoine musical en région (PMR), portion française du Répertoire international des sources musicales (RISM) ;

- des documents de tous types, décrits selon les ISBD et dont l'informatisation en MARC était, de fait, logique (estampes, documents audiovisuels, manuscrits unitaires, etc.).

\section{MARC-TEI : FORMAT DE DESCRIPTION DES INCUNABLES}

Le signalement des incunables est spécifique, parmi celui des livres anciens, car la description des variantes d'édition conduit parfois à des notices longues et complexes, dont le MARC a du mal à rendre compte. Le schéma XML MARC-TEI, dérivé de MarcXchange ${ }^{11}$, a été mis au point pour permettre d'informatiser les notices existantes sans perte de données, tout en répondant à l'exigence d'interopérabilité avec les catalogues

10. Le MARC est généralisé pour les catalogues, mais pas nécessairement pour les bibliographies. The Universal Short Title Catalogue (USTC) : < http://www.ustc.ac.uk/ >, dédié aux livres des $\mathrm{xv}^{\mathrm{e}}$ $\mathrm{XVI}^{\mathrm{e}}$ siècles, et qui recense entre autres 85000 éditions françaises est structuré en base de données. En revanche, l'informatisation en MARC de l'Inventaire chronologique des éditions parisiennes du $\mathrm{XvI}^{e}$ siècle de Renouard (Paris : Service des travaux historiques de la Ville de Paris) est en cours et les notices seront bientôt disponibles dans le catalogue général de la BnF.

11. Pour MarcXchange, cf. infra. 
internationaux ${ }^{12}$. Selon ce format, la structure principale d'une notice bibliographique est en MARC ; l'inclusion de séquences TEI permet d'incorporer les citations longues (descriptions à coupures de lignes), et d'identifier précisément incipits, colophons, rubrication, etc. Par ailleurs, le contexte XML permet une gestion adéquate des caractères typographiques rares et du formatage.

\begin{abstract}
La TEI
La TEI offre un cadre pour développer des DTD ou des schémas permettant un balisage précis de textes ${ }^{13}$. Dans certains pays, elle est utilisée pour décrire les manuscrits. Elle est, par ailleurs, utilisée à la BnF pour la description des reliures remarquables de la Réserve des livres rares. Ce sont certains éléments du sous-ensemble de la TEI prévu pour la description de manuscrits qui sont utilisés pour les incunables.
\end{abstract}

Seuls des prototypes de notices MARC-TEI ont été créés pour l'instant, dans le cadre de l'étude de faisabilité menée par le Centre d'études supérieures de la Renaissance pour la conversion rétrospective des Catalogues régionaux des incunables des bibliothèques publiques de France, qui devrait aboutir en 2014. Des développements sont en cours pour que le catalogage des incunables puisse par la suite se faire dans une base nationale unique, via un SIGB adapté ${ }^{14}$. Il est envisagé que le futur catalogue national des incunables en ligne soit accessible dans le CCFr par une interface de consultation dédiée, susceptible d'exploiter toute la richesse du MARC-TEI.

\title{
EAD : MANUSCRITS, ARCHIVES, FONDS STRUCTURÉS
}

Pour la description des manuscrits et des fonds d'archives, et plus généralement des ensembles hiérarchisés de documents, quelle que soit leur nature, le format le plus adapté est l'EAD. Ce format d'encodage est né $\mathrm{du}$ besoin d'informatiser les instruments de recherche archivistiques

12. En particulier l'Incunabula Short Title Catalogue $:<$ http://www.bl.uk/catalogues/istc/index. html $>$.

13. Text Encoding Initiative : < http://www.tei-c.org/ >.

14. Une solution alternative serait celle d'un éditeur XML offrant l'apparence d'un formulaire de saisie. 
traditionnels et de les mettre en ligne. La première version de ce standard a été publiée par la Society of American Archivists en 1998. C'est la DTD EAD 2002, reposant sur le langage XML et entièrement conforme à la norme ISAD(G), qui est actuellement utilisée ${ }^{15}$.

L'EAD comprend 146 éléments, dont la plupart peuvent être qualifiés par des attributs. Outre les éléments de métadonnées, de structuration, de liens, d'indexation et les éléments génériques, on note surtout une trentaine d'éléments d'information spécifique qui correspondent aux principales unités d'information de la norme internationale de description ISAD(G). Tirant parti de l'héritage implicite des données propre à l'arborescence XLM, l'EAD est particulièrement adapté à la description de l'architecture d'un fonds d'archives en séries, sous-séries, dossiers et pièces.

\section{Décrire des manuscrits et archives : ISAD(G) et DeMarch}

Le catalogage des manuscrits et des documents d'archives a longtemps souffert de l'absence de règles unifiées. La norme internationale de description archivistique ISAD(G), destinée aux centres d'archives, n'a guère été mise en œuvre dans les bibliothèques. Ce vide normatif a été comblé en 2010 par la publication, sous l'égide de l'AFNOR, de Description des Manuscrits et fonds d'Archives modernes et contemporains en bibliothèque - DeMArch ${ }^{16}$. Bien que les recommandations DeMArch n'aient pas le statut de norme, elles en ont toute la valeur scientifique. Il s'agit d'une déclinaison d'ISAD(G) adaptée aux bibliothèques françaises, qui rappelle les principes à respecter lors de la description de documents d'archives (description du général au particulier, indication des informations spécifiques au niveau de description, lien hiérarchique entre unités de description, non-répétition des informations) et qui détaille la manière d'exprimer les différents éléments d'information (identification, contexte, contenu et structure, communication et utilisation des documents, acquisition et évaluation, etc.).

15. Cf. < http://www.archivists.org/saagroups/ead/ >. L'EAD est actuellement en cours de refonte et une nouvelle version, sous forme de schéma XML, doit voir le jour à l'été 2013.

16. Association française de normalisation. Commission de normalisation Modélisation, production et accès aux documents, Description des manuscrits et fonds d'archives modernes et contemporains en bibliothèque : DeMArch [en ligne], 2010, < http://www.bivi.fonctions-documentaires. afnor.org/livres-blancs/recommandation-de-description-des-manuscrits-et-fonds-d-archives >. Pour les manuscrits médiévaux, il n'existe pas de document similaire. On se référera aux pratiques de description de la BnF. 
Contrairement au MARC, la mise en œuvre de l'EAD dans les bibliothèques s'est faite d'abord par des opérations massives de rétroconversion, plus que par du catalogage, qui se heurtait à l'absence d'outils ergonomiques. L'informatisation en EAD du Catalogue général des manuscrits des bibliothèques publiques de France (CGM) et du Répertoire des manuscrits littéraires français $d u x^{e}$ siècle (PALME) qui concernait environ 800 établissements, comme celles des divers catalogues de manuscrits de la $\mathrm{BnF}$, a créé en peu de temps (2005-2010) une grande masse de données qui sont maintenant accessibles dans trois catalogues : BnF archives et manuscrits, Catalogue en ligne des archives et des manuscrits de l'Enseignement supérieur (Calames) et le $\mathrm{CCFr}^{17}$.

\section{Travailler en EAD}

Chaque bibliothèque est encouragée à récupérer les données du CGM et de PALME correspondant à ses documents et à les mettre à jour. Afin de garantir l'homogénéité des données dans les catalogues collectifs, le programme de mise à jour et d'enrichissement du CGM est coordonné par la BnF et par l'ABES ${ }^{18}$. Un guide de correction en cours de rédaction apportera les indications nécessaires. Un établissement peut procéder à cette mise à jour de multiples manières :

- correction des données rétroconverties ;

- catalogage de nouveaux fonds en EAD ;

- conversion de catalogues existants sous traitement de texte, tableur ou au format MARC ;

- réutilisation de données PALME au sein des fichiers du CGM pour créer des notices conformes à la structure des fonds.

Il n'existe pas encore d'intégration adéquate entre les outils EAD et les modules de catalogage et les OPAC des SIGB. Pour rédiger des descriptions en $\mathrm{EAD}$, il convient de créer un document dans un éditeur XML et

17. Cf. BnF archives et manuscrits < http://archivesetmanuscrits.bnf.fr/ > ; Calames < http://www. calames.abes.fr/pub/ > pour les données de l'Enseignement supérieur ; le CCFr < http://ccfr.bnf. fr > pour celles des bibliothèques des collectivités territoriales.

18. Depuis 2008, la BnF offre, deux fois par an, une formation à l'EAD et à la mise à jour du CGM et PALME. 
de lui associer la DTD EAD ${ }^{19}$. L'éditeur peut, au premier abord, paraître déroutant. Il est nécessaire de suivre une formation à XML pour bien appréhender ce langage (imbrication des balises, héritage des informations, qualification par les attributs) et de prévoir un temps d'adaptation pour manipuler correctement l'éditeur. On peut toutefois associer au document XML une feuille de style qui donne à l'éditeur l'apparence d'un formulaire de saisie. La consultation de la documentation officielle (Description archivistique encodée, Dictionnaire des balises) ${ }^{20}$ est absolument indispensable afin de connaître le détail du format ; elle doit être complétée par celle du Guide des bonnes pratiques de l'EAD en bibliothèque, dont la publication est imminente ${ }^{21}$.

\section{Fonctionnalités XML}

Pour établir des requêtes et manipuler les données, il faut apprendre à tirer parti des fonctionnalités du langage XML (XPath, XSL). Il est ainsi possible de créer des extractions ciblées, de procéder à des ajouts automatiques dans les données (indexation, mentions de provenance), de créer de nouveaux instruments de recherche à valeur ajoutée, de se munir d'outils d'analyse des collections, ou bien encore de créer des métadonnées pour une bibliothèque numérique. En établissant une correspondance de champ à champ, on peut créer des notices en Dublin Core $^{* 22}$ pour exposer les descriptions via OAI-PMH ${ }^{23}$.

La mise en ligne de catalogues EAD peut se faire par une simple sortie HTML ou PDF créée par un programme de transformation. Pour obtenir une solution plus satisfaisante pour la navigation et la recherche, il est nécessaire d'utiliser un logiciel spécifique. L'outil libre Pleade est celui qui

19. Les éditeurs les plus fréquemment utilisés sont <oXygen/>, XMetal et XML Mind. Le fichier EAD.DTD et ses fichiers complémentaires sont téléchargeables sur le site officiel de l'EAD < http://www.loc.gov/ead/ >. La BnF a développé l'outil PiXML pour le catalogage en EAD. Calames est également doté d'une interface de catalogage.

20. La traduction en français est disponible parmi la documentation relative à l'EAD sur le site des Archives de France : < http://www.archivesdefrance.culture.gouv.fr/gerer/classement/normesoutils/ead/ $>$.

21. Cf. < http://www.bonnespratiques-ead.net/ >.

22. Cf. infra p. 129.

23. C'est ainsi que sont créées, par exemple, les notices descriptives des manuscrits dans la bibliothèque numérique Gallica < http://gallica.bnf.fr/ >. 
est le plus largement employé par les bibliothèques pour mettre en ligne leurs instruments de recherche ${ }^{24}$.

Il est possible qu'à l'avenir l'EAD soit étroitement associé au schéma EACCPF (Contexte archivistique encodé : collectivités, personnes, familles), qui est une transposition en XML des prescriptions de la norme archivistique internationale relative aux autorités collectivités, personnes et familles ISAAR (CPF). Le format EAC-CPF a vu le jour en $2010^{25}$, et n'est encore que très peu utilisé dans les bibliothèques. Pour l'instant, les interfaces de catalogage EAD dans lesquelles des développements ont été effectués pour gérer les autorités (Calames, PiXML) font appel aux notices d'autorités en MARC, mais EAC-CPF a été conçu pour être parfaitement compatible avec l'EAD et pourrait offrir une alternative, en ouvrant notamment vers des projets de fichiers internationaux d'autorités ${ }^{26}$.

\section{INTEROPÉRABILITÉ}

\section{FRONTIÈRES ET UTILISATION CONJOINTE}

La coexistence de modèles de données hétérogènes peut troubler. Utiliser plusieurs formats est délicat pour une bibliothèque, car cela implique un surcoût en termes de formation et d'investissements informatiques. Le MARC jouit d'un avantage certain : il est déjà très largement présent dans les bibliothèques, convient à de nombreux types de document et permet la gestion des autorités. Mais il importe de ne pas se priver des apports des autres formats, et au contraire de tirer le meilleur parti des atouts de chacun. L'EAD est loin d'être aussi opérant que le MARC pour la description bibliographique des imprimés, mais il permet de rendre clairement l'organisation de fonds et d'ensembles structurés. Or montrer la structuration d'un ensemble de documents, quelle que soit leur typologie, apporte une information précieuse à l'usager, qu'il s'agisse d'un fonds d'écrivain

\footnotetext{
24. Cf. < http://pleade.com/ >.

25. Site officiel : < http://eac.staatsbibliothek-berlin.de/ >. La traduction en français du dictionnaire des balises de l'EAC-CPF est parue en $2012:$ < http://www.archivesdefrance.culture.gouv.fr/ gerer/classement/normes-outils/eac/ >.

26. Cf. le projet Social Networks and Archival Context (SNAC) $:<$ http://socialarchive.iath.virginia. edu/index.html >.
} 
contenant à la fois archives, manuscrits et imprimés, ou d'un fonds d'architecte composé de dossiers, brochures, plans et photographies. L'utilisation conjointe de MARC et d'EAD permet de décrire l'ensemble en EAD, en ajoutant, au niveau approprié un éventuel renvoi vers une notice MARC plus complète. Parallèlement, la notice MARC peut renvoyer à l'inventaire $\mathrm{du}$ fonds en EAD.

L'EAD est maintenant utilisé au-delà des manuscrits et documents d'archives. La BnF l'emploie pour le catalogage de certains documents spécialisés (collections des Arts du spectacle, ensembles de documents audiovisuels) ; la bibliothèque municipale de Dijon l'a utilisé pour sa collection de menus et l'Institut mémoires de l'édition contemporaine (IMEC) le prévoit pour ses fonds de bibliothèques d'éditeurs ${ }^{27}$. Une concertation nationale paraît particulièrement souhaitable en ce qui concerne le format de description le mieux adapté pour les fonds et collections d'images fixes. L'usage traditionnel du MARC est remis en cause par divers projets de traitement en EAD et par le choix de l'ABES de décrire de larges collections de documents photographiques dans l'outil EAD Calames.

\section{PORTAILS MULTI-FORMATS}

Il existe un intérêt stratégique à orienter ses utilisateurs vers un unique point d'accès aux collections. Pour l'usager, peu importe le format de description, seules comptent la pertinence du résultat de recherche et la complétude de la description. La solution consiste à se doter d'un portail qui fédère les catalogues de formats différents et offre des critères de recherche basés sur des index communs alimentés par ces sources hétérogènes.

Le CCFr, qui a vocation à localiser l'ensemble des documents patrimoniaux et spécialisés de France, présente l'exemple d'un tel portail puisqu'il offre à la fois :

- un accès unique - mais restreint à certains éléments de description - à l'ensemble des données, qu'elles soient en MARC, en EAD, en Dublin Core ou dans un autre format ; 
- des interfaces dédiées permettant de consulter des index particuliers ou d'exploiter toute la richesse d'un format (Base Patrimoine, documents musicaux, manuscrits) ${ }^{28}$.

\section{PRÉSENCE SUR LE WEB ET PERSPECTIVES D'ÉVOLUTION}

Le paysage des formats documentaires et de leur transcription informatique est en pleine évolution. La nécessité des échanges de données sur le Web impose une interopérabilité technique à travers le langage XML. Les formats MARC peuvent être exprimés en XML : Unimarc l'est ainsi par l'intermédiaire du schéma MarcXchange ${ }^{29}$. Techniquement, il est désormais facile de convertir les données MARC en d'autres formats, ou d'utiliser MARC conjointement avec d'autres formats au sein de schémas XML (ainsi pour MARC et TEI dans les descriptions d'incunables).

Le Web impose également aux bibliothèques de s'intégrer dans un écosystème où les modèles de données sont multiples, et où le MARC n'a aucune prépondérance particulière. Jusqu'à présent, les données catalographiques exposées sur le Web sont majoritairement des données légères en Dublin Core.

\section{Dublin core}

Le Dublin Core est un ensemble de recommandations pour la description bibliographique minimale de documents ou objets présents sur le Web. Il joue un rôle fondamental dans le partage de métadonnées pour les catalogues et les bibliothèques numériques, via le protocole OAI-PMH. Ce format générique et simplifié ne gère ni la hiérarchie des informations ni les autorités. Sa concision fait sa robustesse mais l'hétérogénéité du contenu des champs peut nuire à l'interopérabilité. Il ne s'agit pas d'un format de description mais d'échange : on décrit

28. Xavier Borda, Véronique Falconnet, Jérôme Sirdey, « Le catalogue collectif de France, pour quoi faire ? », Bulletin des bibliothèques de France, 2011, t. 56, n 2, pp. 71-76. [En ligne] : < http:// bbf.enssib.fr/consulter/bbf-2011-02-0071-001 >. Le portail des manuscrits du Consortium of European Research Libraries (CERL) < http://cerl.epc.ub.uu.se/sportal/?lang=fr > est un autre exemple d'accès à des données en plusieurs formats.

29. Cf. < http://www.loc.gov/standards/iso25577/ISO_DIS_25577_E_.pdf >. Parmi les nouveaux formats, on note MODS, qui correspond à une version XML simplifiée du MARC. La bibliothèque municipale de Lyon l'utilise pour sa bibliothèque numérique. Le format MADS est utilisé en complément de MODS pour les données d'autorités. 
les documents dans un format riche (MARC, EAD) à partir duquel on extrait des métadonnées légères en Dublin Core.

Mais on ne peut se contenter de n'exposer sur le Web que des données " dégradées ». L'avenir des données catalographiques, dans toute leur richesse, est au contraire promis sur le Web. L'adoption du code de catalogage RDA par les bibliothèques françaises est actuellement à l'étude. Dans cette optique, le format MARC est appelé à disparaître au profit d'un nouveau format fondé sur XML et RDF, et qui mettra en œuvre les spécifications fonctionnelles des notices bibliographiques (FRBR) ${ }^{30}$. Cela induira une ouverture vers des modèles éclatés de l'information, dans lesquels ce sont les relations entre les différentes unités d'information qui importent. Dans cet environnement du "Web de données ", les informations sont structurées et auto-documentées, afin d'être exploitées de multiples manières par divers acteurs.

Pour les responsables de fonds patrimoniaux, cela exigera une nouvelle approche du signalement. Lorsque l'ensemble des collections aura été décrit, les chantiers de l'avenir verront un double mouvement de retour vers les documents (enrichissement des notices par des données d'exemplaires : reliure, provenance, notes manuscrites) et d'ouverture vers des bases externes (récupération d'indexation matière, liens vers des réservoirs d'autorités internationaux, ajouts d'identifiants internationaux des œuvres et de leurs expressions, liens vers les documents numérisés dans des bibliothèques numériques externes). Un tel enrichissement des notices, "livre et souris en main ", permettra de répondre aux exigences du Web en matière de navigation fluide, d'immédiateté des rebonds, de regroupements intelligents d'information, de filtres pour éviter le bruit et de pistes offertes à l'usager pour éviter le silence. Signalement et valorisation se rapprocheront ainsi par l'éditorialisation des catalogues par laquelle les bibliothécaires inventeront de nouvelles formes de visualisation des collections ${ }^{31}$.

30. Cf. Groupe technique sur l'adoption de RDA en France : < http://rda-en-france.enssib.fr/ > .

31. Le projet data.bnf.fr < http://data.bnf.fr/ > donne, dès à présent, un aperçu des regroupements possibles d'information mais les possibilités de visualisation pourront être poussées beaucoup plus loin. 


\section{PARTiE int}

\section{GÉRER UN PROJET PATRIMONIAL}

1. METTRE À NIVEAU UNE BIBLIOTHĖQUE par Carole Jacquet

$++++++++++++++++++++++++++++++++++++++++++++$

2. RÉVEILLER UN FONDS PATRIMONIAL par Noëlle Balley

$++++++++++++++++++++++++++++++++++++++++++++$

3. INSCRIRE SON PROJET DANS LES POLITIQUES PATRIMONIALES NATIONALES ET RÉGIONALES par Thierry Claerr 


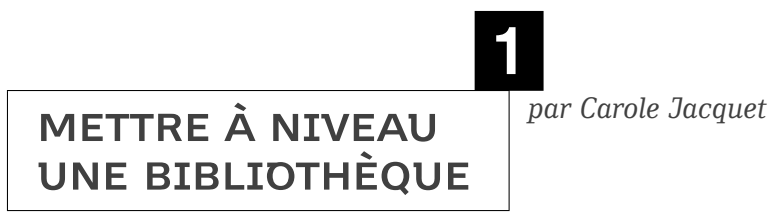

La bibliothèque patrimoniale du Centre culturel irlandais (CCI) a longtemps été fermée au public. Les ressources n'étaient pas consultables en raison d'un empoussièrement conséquent des ouvrages, du degré de dégradation avancé de nombreux volumes et de l'absence de catalogue informatisé.

Un important chantier de préservation des collections patrimoniales a été mis en œuvre entre 2006 et 2011. Le but : réhabiliter les fonds et permettre l'ouverture aux chercheurs pour la consultation.

Mais seul-e ou presque face à un fonds patrimonial, il n'est pas évident de savoir comment s'y prendre : par où commencer ? à qui s'adresser pour des conseils, des expertises, de l'aide ? quels budgets pour quels projets ? Ce qui suit est une méthode qui s'appuie sur l'expérience de remise à niveau de la bibliothèque patrimoniale du CCI.

\section{PAR OÙ COMMENCER ? DÉFINIR LES PHASES DU CHANTIER}

++++++++++++++++++++++++++++++++++++++++++++++++++++++++++++++++++++++++

Prendre contact avec les institutions référentes (BnF, MCC, DRAC...), rencontrer des responsables de fonds patrimoniaux, des experts en restauration, conservation préventive et ingénierie documentaire est un bon début. Leur présenter les fonds, recueillir leur avis permet de :

- dresser un premier état des lieux ;

- définir les priorités ;

- dessiner le contour des grandes actions à mener.

$\mathrm{Au}$ CCI, le projet a été découpé comme suit :

- signalement : conversion rétrospective du catalogue papier ;

- remise en état : campagne de maintenance (dépoussiérage, cirage, constitution d'une base de données) et de conservation (restauration sur site et envois en ateliers extérieurs) ; 
- amélioration des conditions de conservation : audit environnemental ;

- valorisation : numérisation, bourses de recherche, expositions, visites, publications.

Une fois les phases de chantier définies, reste la mise en œuvre.

La mise à niveau d'une collection nécessite des connaissances dans des domaines variés (informatique, restauration, conservation, numérisation...) et des moyens humains. Outre les deux membres de l'équipe des ressources documentaires du CCI, nos chantiers ont nécessité le renfort d'une dizaine de professionnels. Afin de contourner la difficulté que représente un grand nombre d'embauches pour une structure, il est possible d'avoir recours à des prestataires extérieurs pour des missions clés en main, le détachement d'agents et l'encadrement de travaux spécialisés. Les pôles associés de la BnF peuvent également bénéficier de l'expertise de ses différents départements.

Tous les prestataires ont été sélectionnés selon une grille de dépouillement comparant :

- la compréhension du projet ;

- les prestations : planning, lieu de réalisation, assurances, respect des exigences, tests, méthodes et outils utilisés ;

- les équipes et l'expérience de la société : CV des intervenants, nombre d'employés, chiffre d'affaires, références clients ;

- les coûts et délais : coûts négociables, coûts fixes, modes de livraison...

\section{SIGNALER}

L'informatisation constitue le premier volet de la mise à niveau des fonds, préalable à l'exploitation ultérieure des collections.

La rétroconversion peut se faire en interne ou être externalisée, tout dépend : 
- des délais impartis ;

- des moyens humains ;

- des moyens financiers ;

- de la qualité du fichier existant.

À titre d'exemple, le traitement des 8000 notices de la collection du CCI a été externalisé pour un budget de $15000 €$ TTC. Le projet s'est déroulé sur neuf mois (de l'appel d'offres à la livraison). La rétroconversion a été soutenue par la BnF, dans le cadre d'une convention de pôle associé. L'équipement informatique a été subventionné par la DRAC Île-de-France.

Le signalement des collections permet de doter la bibliothèque d'un catalogue en ligne et d'intégrer un réseau en participant à des catalogues collectifs français et étrangers (Catalogue collectif de France, English Short Title Catalogue...), ce qui contribue à la visibilité et au rayonnement des collections.

Pour intégrer le CGM, les archives et manuscrits doivent être décrits en $\mathrm{EAD}^{1}$. Au CCI, cet encodage a été réalisé en interne : après une formation à l'EAD organisée pour les pôles associés, l'une de nos collègues a travaillé en étroite collaboration avec le référent EAD de la BnF.

Les soutiens financiers des partenaires ne sont donc pas les seules aides disponibles : des appuis techniques de grande valeur sont aussi possibles.

L'assistance d'un cabinet de conseil en ingénierie documentaire peut aussi être une solution pour renforcer une expertise technique ou tenir des délais serrés. Au CCI, 6 jours de prestation ont été commandés pour :

- une relecture du cahier des charges ;

- une aide à la validation des spécifications techniques ;

- une aide à la correction des anomalies.

Bien que cela ait un coût élevé, une prestation d'assistance est parfois plus simple à négocier qu'une embauche. Mais s'il est important d'obtenir des budgets et des moyens humains, il s'avère tout aussi indispensable de négocier des délais adéquats.

1. Voir plus haut l'article de Florent Palluault : « Décrire et signaler des documents patrimoniaux : enjeux, formats, perspectives », pp. 123-127. 


\section{REMETTRE EN ÉTAT}

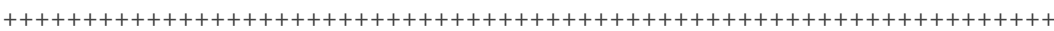

Après avoir signalé les collections, il faut les rendre accessibles. Le deuxième volet de mise à niveau des fonds patrimoniaux porte sur la remise en état.

\section{Campagne de maintenance}

La campagne de maintenance peut s'articuler autour des opérations suivantes :

- collecte des données : constat d'état, repérage des altérations et consignation dans une base de données ;

- dépoussiérage : reliure, premiers et derniers feuillets ;

- cirage de certaines reliures en cuir ;

- banderolage* de reliures présentant des éléments qui risqueraient de se détacher.

Recourir à des techniciens en préservation plutôt qu'à une entreprise de dépoussiérage est pertinent : manipulation adéquate, sensibilité aux enjeux de la conservation, capacité à repérer et décrire des dégradations en vue d'alimenter la base de données. On peut ainsi bénéficier d'un état des lieux complet du fonds, un gain de temps considérable pour une campagne de restauration ultérieure.

Notre campagne de maintenance a été montée en collaboration avec une société de conseil en préservation des biens culturels qui a recruté et détaché trois techniciens en préservation.

78 semaines / homme ont été consacrées à la maintenance des 8000 volumes de la collection.

Pour s'engager sur un calendrier de réalisation viable, les projections et objectifs quantitatifs à atteindre peuvent être établis sur la base de 6 heures de traitement effectif des collections par jour (sur les 7 heures en moyenne d'une journée de travail), afin de tenir compte du temps quotidien nécessaire à l'installation, au rangement, aux éventuelles réunions de coordination... 
L'externalisation du programme de maintenance permet de gagner en simplicité sur le plan administratif. En revanche, une prestation "clé en main » a un coût plus élevé qu'un programme avec des embauches en direct. Outre le salaire des techniciens, il faut prévoir la marge du prestataire, les journées de suivi et de coordination de ce dernier. Par ailleurs, il ne faut pas négliger la disponibilité requise en interne pour suivre le chantier : le consultant n'étant présent que ponctuellement ( $1 / 2$ journée par semaine par exemple), le chef de projet sur site peut être très sollicité. Enfin, un programme de dépoussiérage étant répétitif et peu valorisant pour les équipes en charge, la question de la motivation est importante à prendre en compte, en offrant par exemple des salaires un peu plus élevés ou en variant les tâches.

\section{Campagne de conservation}

Petites réparations sur site - capitaliser sur les forces en présence Pour diversifier les activités des techniciens en préservation, tout en poursuivant la réhabilitation des fonds, une campagne de petites réparations peut leur être confiée, sous la supervision d'un restaurateur.

$\mathrm{Au}$ CCI, après quelques journées de formation complémentaires, ils se sont attelés à la consolidation de coins, chants et cuirs pulvérulents, à la réparation de coiffes et mors, à la retouche de zones consolidées. La moyenne de leurs interventions n'excédait pas 40 minutes par ouvrage, uniquement sur les petits formats. Si la quantité de documents traités s'est révélée peu significative, ce programme était valorisant pour les équipes.

\section{Restauration sur site - faire appel à des restaurateurs indépendants} Certains ouvrages nécessitent des traitements plus poussés que de simples petites réparations. Avec du matériel et un espace dédié, on peut entreprendre certaines restaurations sur place.

Nous avons fait appel à trois restaurateurs indépendants qui, après un audit pour définir les priorités de traitement, ont eu pour objectif de restaurer des ouvrages dans la perspective d'une consultation : rattachement de plats ou d'éléments détachés, création de nouveaux plats, renfort de coins, mors, coiffes, tranchefiles, sur tout type de formats. 
Afin de traiter un maximum d'ouvrages dans le budget et les délais impartis, nous avons décidé de ne pas restaurer l'intégralité d'un volume, mais uniquement les dégradations qui menaçaient de s'aggraver lors des manipulations. Seuls les ouvrages nécessitant moins de 2 heures de restauration étaient traités sur site ; 860 ouvrages ont été restaurés en 400 jours / homme.

\section{Restauration en atelier extérieur - nécessaire dans certains cas}

Les documents rares, les très grands formats, les ouvrages présentant des dégradations importantes ou nécessitant une reliure de conservation seront plutôt envoyés en atelier.

Des partenariats peuvent également être noués avec des écoles de restauration. Une quarantaine de nos ouvrages ont ainsi été restaurés à moindre coût par des élèves de l'Institut national du patrimoine (INP) et le Master de conservation-restauration de Paris I.

Toutes filières confondues, 1690 ouvrages ont pu être remis en état lors du chantier.

À titre indicatif la moyenne des coûts de restauration par ouvrage s'est élevée à $590 € \mathrm{HT}$ en atelier extérieur (volumes présentant des dégradations importantes, nécessitant des traitements poussés) et à $120 €$ HT sur site (ouvrages nécessitant des interventions légères ou de petites réparations). Dans les deux cas, le budget s'entend matériel inclus. Ces chiffres sont à considérer avec précaution, puisqu'ils correspondent au contexte particulier de notre projet : des restaurations minimalistes, au papier japonais, stabilisant uniquement les dégradations principales menaçant de s'aggraver.

Par ailleurs, si l'on rapporte le coût de la campagne de restauration sur site au nombre d'ouvrages restaurés, on observe une différence significative du coût moyen par volume traité entre la première année de chantier (350 €) et les suivantes (120 €). Pour parvenir à une telle baisse, soit augmenter le nombre d'ouvrages traités dans le volume de jours commandés, nous avons : 
- rédigé une charte définissant les interventions à réaliser ;

- pris en charge l'administration du chantier : photos avant et après traitement, saisies des constats et rapports, préparation des lots, rangement... ;

- capitalisé sur l'expérience acquise par les restaurateurs ;

- assuré un suivi du chantier via des réunions hebdomadaires.

Un projet sur site implique souvent de coordonner différentes activités et soulève, plus largement, la question du management des équipes. Les prestations des restaurateurs indépendants sont simples à gérer du fait du contrat établi directement entre les deux parties. L'accueil de personnel détaché peut cependant s'avérer moins aisé, notamment si le suivi quotidien du chantier incombe en partie à la structure d'accueil. C'est pourquoi il est important d'établir des règles précises en amont du chantier afin de faciliter la communication entre les différents acteurs du projet et la distinction entre structure cliente et structure d'embauche.

\section{AMÉLIORER LES CONDITIONS DE CONSERVATION}

L'environnement d'un fonds patrimonial est primordial pour sa conservation. Un audit environnemental doit porter sur trois axes d'analyses principaux :

- climat (mesures thermo-hygrométriques, polluants gazeux...) ;

- conditions de stockage (maintien des ouvrages, mesures d'empoussièrement...) ;

- éclairage (mesures d'éclairement, simulation numérique des temps d'irradiation des volumes...).

Notre audit a été confié à un cabinet de conseil en préservation des biens culturels (10 jours de prestation). Cela ne dispense pas d'une surveillance thermo-hygrométrique permanente des locaux, mais, en cas de conditions inadaptées, le rapport d'expertise peut avoir du poids pour convaincre les décideurs, alerter les tutelles et obtenir des moyens. 
Un audit peut être complété par une sécurisation des collections :

- mise en place d'un plan d'urgence : achat de matériel, aménagement d'un local, formation des équipes ;

- installation d'alarmes ou vidéo-surveillance ;

- acquisition de vitrines sécurisées à l'hygrométrie contrôlée.

Au-delà des considérations liées à la préservation des fonds, les questions relatives aux conditions de travail des équipes sont importantes à anticiper : climat, lumière, voire solitude que peuvent induire de tels projets et leur impact sur les délais. L'une des difficultés rencontrées lors du chantier a été liée à l'absence de chauffage dans notre bibliothèque datant du XviII ${ }^{\mathrm{e}}$ siècle. Le calendrier du projet a dû être revu en conséquence, pour limiter la présence sur site des équipes au printemps et à l'été.

\section{VALORISER}

Le dernier volet de la mise à niveau porte sur la valorisation des collections, déclinée en plusieurs axes.

\section{Numérisation}

La numérisation permet de se constituer une vitrine, tout en facilitant le rayonnement des fonds et leur accessibilité. Définir les priorités de numérisation en fonction de son contexte : trésors rares et précieux, pièces fragiles, documents souvent consultés ou susceptibles de l'être... puis déterminer les critères de résolution, prise de vue, mode couleur ou niveaux de gris.

Pour favoriser la visibilité des documents numérisés, multiplier les points d'entrée en les signalant dans des bibliothèques numériques : Patrimoine numérique, World Digital Library, Gallica ${ }^{2} \ldots$ 


\section{Bourses de recherches}

L'attribution de bourses de recherche valorise les collections tout en améliorant la connaissance scientifique du fonds. Dans tous les cas, pensez à définir :

- la durée et les dates de remise des travaux ;

- les indemnités, les modalités d'hébergement et de transport ;

- les sujets (on peut aussi laisser le soin au candidat de présenter un projet) ;

- les livrables : mémoire, exposition, conférence...

Effectuez la sélection selon la pertinence du sujet, la taille du corpus, la solidité du candidat, les perspectives de publications... Au CCI, des bourses de $1000 €$ sont offertes pour un mois.

\section{Expositions}

Des expositions peuvent être montées à peu de frais en s'appuyant sur la documentation produite lors des chantiers : photos avant/après, suivi des campagnes de dépoussiérage et restauration, etc. Cela constitue une mémoire des projets, valorise les équipes et donne à voir l'envers du décor au public. Également, des expositions peuvent être tirées des travaux de chercheurs-boursiers.

Le budget nécessaire pour exposer une dizaine d'ouvrages fut de moins de $3000 €$ (hors acquisition des vitrines) incluant une bourse pour l'adaptation du mémoire d'un chercheur, un habillage graphique et l'impression d'une dizaine de panneaux couleur au format A3.

\section{visites publiques, partenariats et communication}

Lors d'une campagne de valorisation des collections, un rôle nouveau de médiateur se dessine :

- organisation de visites ;

- partenariats ;

- rédaction d'articles pour la presse professionnelle ou les listes de diffusion ; 
- création de cartes postales, marque-pages ou plaquettes de communication.

Si plusieurs de ces actions ne nécessitent pas ou peu de budget, elles peuvent en revanche permettre d'en obtenir en sensibilisant des décideurs ou mécènes à ses projets.

La mise à niveau d'une collection patrimoniale peut prendre plusieurs années. Il convient de prévoir des renforts humains pour tenir les délais mais également de faire preuve de polyvalence pour réussir à passer d'activités relationnelles (réunion de chantier) à des contrôles minutieux demandant calme et rigueur (rétroconversion). Un important travail de préparation, suivi et coordination est à prévoir et la maîtrise rapide du vocabulaire des différents secteurs (informatique, restauration, numérisation...) est précieuse pour dialoguer efficacement.

Les contextes sont différents selon les institutions mais s'informer, s'entourer, aller voir ce qui se fait ailleurs permettra de nourrir une réflexion et d'adapter sa stratégie en fonction des caractéristiques des dispositifs d'aide existants. 


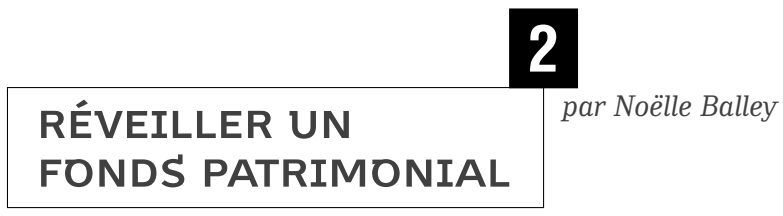

La bibliothèque interuniversitaire $\mathrm{Cujas}^{1}$ pour les sciences juridiques, politiques et économiques, héritière de la bibliothèque de la Faculté de Droit de Paris fondée en 1803, est l'une des plus importantes bibliothèques universitaires juridiques en Europe. Sous sa forme actuelle, elle a été créée en 1979 par convention entre les universités Paris 1 Panthéon-Sorbonne et Paris 2 Panthéon-Assas. Elle est Centre d'acquisition et de diffusion de l'information scientifique et technique (CADIST) et pôle associé de la $\mathrm{BnF}$ pour les sciences juridiques. Riche de près de 800000 monographies, thèses et mélanges, 11000 périodiques et des milliers de ressources électroniques, elle offre aux étudiants à partir du L2, aux chercheurs français et étrangers, le plus vaste panorama de la science juridique disponible en France, orienté prioritairement vers la recherche.

\section{UN FONDS CONSTRUIT PAR DES BIBLIOTHÉCAIRES}

Contrairement à la plupart des fonds patrimoniaux des bibliothèques françaises, son fonds ancien n'est pas issu des confiscations révolutionnaires. Bien au contraire, la collection d'origine de la Faculté de Droit de Paris a été dispersée sous la Révolution, et le fonds actuel a été constitué par achats, dons et legs, essentiellement à partir des années 1870. Il s'agit donc d'un fonds construit rétrospectivement par des bibliothécaires, dans le but de reconstituer « le cabinet de travail d'un robin d'Ancien Régime » - telle était, du moins, la doxa transmise jusqu'à présent de bouche à oreille de bibliothécaire. Jusqu'à l'hiver 2011, le fonds était constitué de 12000 volumes imprimés, datant de la fin du $\mathrm{XV}^{\mathrm{e}}$ siècle au début du XIX ${ }^{\mathrm{e}}$ siècle, essentiellement des livres des $\mathrm{XVII}^{\mathrm{e}}$ et $\mathrm{XVIII}^{\mathrm{e}}$ siècles, 7 incunables, quelques ouvrages précieux postérieurs à 1800 et quelques périodiques. Le quart de ce fonds provient de pays étrangers : Allemagne, Pays-Bas,

1. < http://biu-cujas.univ-paris1.fr >. 
Angleterre et Italie. Particulièrement riche, il comprend $25 \%$ d'ouvrages non référencés à la $\mathrm{BnF}$. La réserve conserve également des cours et notes de cours manuscrits, ainsi qu'une partie des archives de la Faculté de Droit. Les grands courants du droit sont représentés : droit civil, criminel et canonique, pratique du droit ancien ou nouveau (après 1789), coutumiers, œuvres des grands jurisconsultes français et étrangers.

Il n'existe pas de service du livre ancien à Cujas : les acquisitions sont gérées par le département des monographies, le catalogage par celui du traitement documentaire, la magasinière chargée de la maintenance des collections et de l'accueil des lecteurs est rattachée au département des services aux publics, la numérisation sollicite à parts égales des collègues des monographies et du département informatique. Si cette situation présente l'avantage d'instiller un peu de patrimoine dans de nombreux services de la bibliothèque, elle ne constitue pas pour autant un modèle que l'on puisse recommander. Depuis juillet 2010, un conservateur, rattaché au département des monographies, assume la coordination scientifique du fonds et celle du programme de numérisation.

\section{DES DÉBUTS DOULOUREUX : LeS ANNÉES 1980}

Les 12000 ouvrages antérieurs à 1810 ne furent extraits des magasins que dans les années 1980, à la suite d'un important dégât des eaux et d'un vol spectaculaire (les reliures, vidées de leur contenu, ayant été soigneusement laissées en place). Installé sommairement dans des magasins où les lecteurs devaient travailler sans lumière naturelle, au milieu des rayonnages, sur de petites tables d'écolier, le fonds, confié à une magasinière à mi-temps, était exploité par un public confidentiel, mais hautement exigeant, d'enseignants chercheurs et de doctorants en histoire du droit, sur des horaires restreints à quatre demi-journées par semaine.

Il était évident dès le départ que ce patrimoine austère, constitué de textes juridiques anciens peu accessibles au commun des mortels, quasiment dépourvu d'illustrations et en piètre condition physique, ne pouvait s'adresser qu'à un public de spécialistes. Rien ne laissait présager toutefois qu'il connaîtrait en quelques années l'amorce d'une renaissance, encore modeste, mais bien entamée. 


\section{LES DÉBUTS DE LA RENAISSANCE : LE PROGRAMME DE NUMÉRISATION}

La première étape de cette renaissance fut le début du programme de numérisation de la bibliothèque. Sur la base d'une liste de 436 titres fondamentaux pour la recherche en histoire du droit, dressée par deux professeurs, la désormais célèbre " liste Pfister Roumy ", la bibliothèque a développé en quatre ans une chaîne de numérisation, conçue et, jusqu'il y a peu, alimentée entièrement en interne grâce aux compétences du département informatique. Le regard de pédagogues de ces deux enseignants a été un apport fondamental dans la constitution du corpus, destiné en priorité à des étudiants en master ou à des doctorants : si nous avions dû effectuer nous-mêmes la sélection, il est probable que nous aurions privilégié, dans une perspective d'histoire du livre, les éditions originales, ou du moins les plus rares ou les plus anciennes possibles. En choisissant de privilégier des éditions tardives du XviII ${ }^{\mathrm{e}}$ siècle, scientifiquement moins pures, mais bien lisibles, aisément accessibles à des débutants sans compétences paléographiques, les professeurs Pfister et Roumy nous ont évité l'écueil d'un programme construit par des bibliothécaires, à partir de leurs propres centres d'intérêt, mais qui n'aurait pas répondu aux attentes de son public cible.

En son état actuel, la bibliothèque numérique Cujasnum ${ }^{2}$ permet de croiser des recherches sur les métadonnées, sur le plein texte (les documents postérieurs à 1830 sont océrisés), ou dans les tables des matières, et la navigation par listes. Une indexation par facettes géographiques, chronologiques et thématiques a été mise au point, en reprenant le vocabulaire de la liste d'autorités Rameau. Les tables des matières sont intégralement ressaisies à la main, de manière à permettre un accès direct au contenu des documents non océrisés. Une feuilleteuse, en cours de développement, permettra d'ici quelques mois à l'utilisateur d'afficher directement une ou plusieurs pages sans télécharger tout le document.

Le partenariat que nous a proposé la $\mathrm{BnF}$ en 2008, pour assurer un pilotage commun de l'initiative concertée pour la numérisation en sciences juridiques qu'elle souhaitait mettre en place, et le moissonnage de nos

2. < http://cujasweb.univ-paris1.fr/search >. 
données dans Gallica, effectif depuis 2010, ont donné à ce programme de numérisation la visibilité qui lui était indispensable. Grâce à ce partenariat privilégié, des documents appartenant aux collections de la bibliothèque Cujas sont également numérisés sur les chaînes de la BnF et mis en ligne sur Gallica : c'est le cas, notamment, du Bulletin des arrêts de la Cour de cassation.

\section{LA DIVINE SURPRISE : LES TRAVAUX DE MISE EN SÉCURITÉ}

\section{Les années 2000}

La seconde étape fut plus inattendue. Grâce à deux rapports favorables rendus, l'un par la mission hygiène et sécurité de l'université Paris 1 , l'autre par l'IGB, la bibliothèque a obtenu en 2009 les crédits qui lui ont permis de créer, dans le cadre des travaux de mise en sécurité incendie des magasins de la réserve, une petite salle de lecture dédiée au patrimoine, et d'installer des rayonnages mobiles dans deux niveaux de soussols équipés d'un contrôle climatique*. Le déblocage inattendu de ces crédits en cours d'année nous a conduits à organiser, dans des délais très restreints, une mise en conservation sommaire (dépoussiérage, cirage, petites consolidations, banderolage et mise en boîtes) des documents les plus abîmés, grâce au recrutement, à l'été 2009, de trois vacataires relieuses formées à la conservation préventive, et la réalisation, en un temps record, de constats d'état sommaires, avant un déménagement qui impliquait une année de mise au garde-meubles, avec possibilité de consultation sur rendez-vous dans les locaux du prestataire. Le déménagement fut confié à une société spécialisée dans le déplacement des œuvres d'art, le cahier des charges imposant un emballage individuel de chaque document sous le contrôle de la bibliothèque. Ainsi fut réalisé, sous la pression et en quelques semaines, un travail de conservation que l'on aurait à peine envisagé de mener sur plusieurs années...

$\mathrm{Au}$ retour des documents, plusieurs mois furent encore nécessaires à l'aménagement de la salle de lecture et aux réglages de l'installation climatique. Ce n'est qu'à l'automne 2011 que la salle du Patrimoine ouvrit ses portes au public, avec des horaires élargis à une matinée supplémentaire. Confortable et tranquille, cette petite salle, dotée d'un accès informatique 
au portail de la bibliothèque et de quelques usuels qui commencent à trouver leur public, est très appréciée de ses utilisateurs. À cette occasion ont été réalisés, pour servir d'outils de communication, deux jolis signets et un reprint très soigné d'une des raretés du fonds, l'avant-projet de la Constitution de $1791^{3}$. Depuis sa réouverture, la salle du Patrimoine connaît une fréquentation en nette hausse : avec une moyenne de 10 demandes de consultation par semaine sur les trois derniers mois de l'année, elle est déjà très au-delà des 145 lecteurs accueillis en 2008, dernière année de plein fonctionnement sous l'ancien système.

\section{REVISITER SON FONDS PATRIMONIAL}

\section{ENRICHIR LE CATALOGUE}

Restait à déterminer comment seraient utilisés les métrages linéaires gagnés par l'installation des rayonnages mobiles. Après une étude approfondie du catalogue de la bibliothèque - dont la conversion rétrospective venait de s'achever - il a été décidé d'étendre le fonds patrimonial aux documents des deux premiers tiers du XIX ${ }^{e}$ siècle, sans s'interdire d'y introduire ponctuellement des ouvrages postérieurs. Les 15000 volumes antérieurs à 1870 sont progressivement transférés à la réserve. Afin de faciliter l'accès à leur contenu, des liens vers la version numérique, lorsqu'elle existe sur Gallica ou sur toute autre bibliothèque numérique, sont saisis manuellement dans le catalogue local, en attendant la réinformatisation ${ }^{4}$ toute proche de la bibliothèque qui devrait permettre la recherche fédérée sur plusieurs bases. On espère pouvoir, grâce à ce nouvel outil, valoriser le fonds patrimonial en créant une " vue » spécifique sur son catalogue, et en l'enrichissant de liens vers des images numérisées. Par ailleurs, un petit groupe de travail transversal a été créé pour enrichir la base des 4000 signets signalés sur le portail de la bibliothèque ${ }^{5}$ par la création

3. La Constitution française, projet présenté à l'Assemblée nationale par les Comités de constitution et de révision, Paris, Imprimerie nationale, 1791.

4. Le système choisi est Aleph de la société Ex Libris.

5. Onglet « Annuaire des sites » sur le portail de la bibliothèque Cujas : < http://cujas-front. univ-paris1.fr/repons/portal/bookmark?Global=1\&Body=5\&Search_5=2\&MenuItemSelected= cujastheque\&MenuItemPosition $=0 \&$ ShowHttp $=>$. 
d'un onglet spécifique, recensant des sites Web utiles à l'histoire du droit. Grâce aux recherches menées dans ce cadre, et pour améliorer l'indexation de la bibliothèque numérique, des membres de l'équipe acquièrent progressivement une expertise sur les sources de l'histoire du droit.

\section{CONNAÎTRE SON FONDS}

En parallèle, notre connaissance globale du fonds s'enrichit. Les heures passées à explorer systématiquement, année par année, le catalogue de la bibliothèque pour définir les limites chronologiques du fonds patrimonial, puis repérer les ouvrages antérieurs à 1870, ont révélé la richesse inattendue de ses collections dans des disciplines non juridiques, telles que l'histoire générale ou l'économie politique, pour le XVIII ${ }^{\mathrm{e}}$ et la première moitié du XIX ${ }^{\mathrm{e}}$ siècle. Ces fonds jusque-là inexploités, issus d'une politique d'acquisitions beaucoup plus large qu'aujourd'hui, et représentatifs de l'état de l'enseignement dans les Facultés de Droit au XIX ${ }^{e}$ siècle, sont porteurs d'un potentiel de valorisation susceptible de dépasser le cercle restreint des seuls historiens du droit. D'autre part, les quelques fonds d'archives et de manuscrits que conserve la bibliothèque commencent à être inventoriés en vue de leur intégration dans Calames.

\section{SUSCITER L'INTÉRÊT DES ENSEIGNANTS CHERCHEURS}

L'intérêt des équipes de recherche pour les collections patrimoniales de la bibliothèque se développe sensiblement depuis quelques mois. À l'occasion des visites proposées aux professeurs des deux universités de tutelle pour l'ouverture de la salle du Patrimoine, des contacts fructueux ont été noués avec quelques enseignants chercheurs. De manière très inattendue, un fonds rare de 7000 thèses allemandes des $\mathrm{XVI}^{\mathrm{e}}-\mathrm{XVIII}{ }^{\mathrm{e}}$ siècles, jusque-là totalement inexploré, a suscité l'intérêt d'une équipe de recherche, ce qui nous encourage à en entreprendre enfin l'inventaire et le catalogage. Le conservateur a également été sollicité pour participer à une publication consacrée à l'histoire de l'université Paris 1 . À la demande de leurs professeurs, des présentations des ressources numériques utiles à l'histoire du droit sont dispensées depuis deux ans, à chaque rentrée universitaire, aux étudiants du master en histoire du droit de Paris 2, et rencontrent un grand succès. 
La participation d'un conservateur de la bibliothèque aux travaux du groupe Corpus des Enseignants en Droit, Répertoire archivistique et Enquête prosopographique (CEDRE) ${ }^{6}$, qui se consacre à la prosopographie des professeurs de droit, a aussi permis d'éveiller l'intérêt d'un maître de conférences, qui se propose d'étudier, pour un colloque qui se tiendra en mars 2013, l'évolution des manuels de droit à travers les collections de la bibliothèque. Celle-ci est aussi régulièrement sollicitée pour apporter son soutien technique à des programmes de recherche centrés sur l'exploitation de sources numérisées : le programme Droitpol $^{7}$ s'inscrit dans $^{\prime}$ une démarche de recherche en droit constitutionnel, associant la mise en ligne de sources et des séminaires de travail, centrés sur les grands moments de l'histoire constitutionnelle de la France. Le projet Mémoloi, porté par le Centre d'études pour la coopération juridique du CNRS (CECOJI) ${ }^{8}$, sera axé sur l'élaboration des grandes lois sur la sauvegarde du patrimoine. Dans les deux cas, la bibliothèque contribue au projet en mettant ses collections à la disposition du groupe projet, en fournissant les fichiers numériques et en assurant la relecture des OCR.

\section{QUELQUES LEÇONS DICTÉES PAR L'EXPÉRIENCE}

\section{ASSOCIER LES CHERCHEURS EN PRIORITÉ}

Quels enseignements tirer de ces années riches en événements?

D'abord, que le patrimoine a plus que jamais besoin des chercheurs. Seul un enseignant expérimenté pouvait orienter notre programme de numérisation dans un sens qui soit vraiment utile à des étudiants novices. Si le regard du bibliothécaire est irremplaçable pour acquérir une vision synthétique des collections ou résumer un ouvrage en quelques mots clés, la connaissance détaillée des contenus appartient aux chercheurs, qui connaissent nos documents de l'intérieur, puisqu'ils prennent le temps de les dépouiller, et sont à même de poser sur eux un regard réellement

\footnotetext{
6. CEDRE : < http://www.sciences-sociales.ens.fr/-Centre-de-Theorie-et-Analyse-du-.html >.

7. Projet soutenu par l'Agence nationale de la recherche : projet ANR-09-BLAN-0359. Voir le site Web < http://www.droitpolitique.com $>$.

8. CECOJI $:<$ http://www.cecoji.cnrs.fr $>$.
} 
critique. Et force est d'admettre que l'écart entre le socle de connaissances du grand public (et des bibliothécaires...) et ces documents écrits dans des langues anciennes dont nous commençons à perdre les codes d'accès, nécessitera de plus en plus la médiation de spécialistes : l'appropriation du patrimoine écrit par le grand public devra, demain plus que jamais, s'appuyer sur le décodage offert aux bibliothécaires par le travail de la recherche.

\section{MULTIPLIER LES PARTENARIATS}

Ensuite, qu'il faut savoir tirer parti de toutes les occasions et multiplier les partenariats. Nul n'aurait pu prédire en 2008 que le fonds patrimonial de Cujas connaîtrait en trois ans de telles avancées. Le partenariat avec la $\mathrm{BnF}$ nous a permis de sortir notre bibliothèque numérique d'un manque de visibilité qui, compte tenu des programmes de numérisation de masse alors en plein développement, lui aurait sans nul doute porté un grand tort. L'obligation de mettre en sécurité nos magasins a permis la création de la salle du Patrimoine, et l'accession au statut patrimonial des monographies du XIX ${ }^{\mathrm{e}}$ siècle. On sait bien que la numérisation, en particulier, est un levier puissant, qui suscite un vif intérêt (en même temps que beaucoup de fantasmes...) de la part des décideurs, et permet bien souvent d'obtenir enfin les moyens de signaler un fonds ou de restaurer un document. C'est aussi le moyen, pour le bibliothécaire averti, d'apparaître aux yeux du chercheur comme un expert technique dont non seulement la connaissance des fonds, mais aussi les compétences en numérisation, en indexation, en gestion de bases de données, en propriété intellectuelle, seront utiles à son projet.

\section{MAÎTRISER SON FONDS PAR GRANDES ÉTAPES}

Enfin, qu'il faut absolument prendre le temps de très bien connaître son fonds patrimonial, en commençant par les grandes masses, sans se lancer d'emblée dans des chantiers infinis de catalogage fin qui, sans objectif précis, seront abandonnés au bout de quelques mois pour être remplacés par d'autres priorités. Privilégier la vision globale du fonds, acquise par sondages sur les rayonnages et dans le catalogue, avant de passer à l'analyse 
fine de chaque document : mieux vaut une série complète de constats d'état très simplifiés, exploitables informatiquement ${ }^{9}$, que quelques dossiers de restauration minutieusement dressés, sans vision d'ensemble des besoins ni plan pluriannuel. Recueillir soigneusement la connaissance du fonds acquise, au fil des années, par les anciens de la bibliothèque, ces « fureteurs » qui n'ont pas leur pareil pour dénicher des pépites au milieu des rayonnages. Respecter avant tout l'objet livre, comprendre comment il a été fabriqué, pourquoi il s'est dégradé, et pour cela dialoguer longuement avec les relieurs, les restaurateurs, les collègues plus expérimentés. Écouter ses lecteurs, se passionner pour leurs trouvailles, bondir sur leurs suggestions, rebondir sur leurs critiques. Et surtout, prendre le temps de se promener longuement dans ses magasins, de repérer les tuyaux et les taches sur les murs, d'ouvrir les boîtes, mais aussi - c'est complémentaire - d'explorer très minutieusement son catalogue, en multipliant les recherches croisées, par tranche chronologique, par thématique, par pays, par langue. Cet exercice nous a été, de loin, le plus utile pour acquérir en si peu de temps une meilleure maîtrise de ce fonds que, finalement, nous ne connaissions jusque-là que par ouï-dire.

\section{SAVOIR RACONTER LA COLLECTION PATRIMONIALE}

Dernière leçon, le patrimoine, même le plus austère en apparence, n'est pas un milieu hostile. Il suscite l'intérêt d'un public, souvent passionnant, toujours passionné... et aussi, si l'on sait s'y prendre, celui d'interlocuteurs qui peuvent s'avérer fort utiles au bibliothécaire : une visite guidée, personnalisée, offerte au service des marchés de la collectivité, à l'agent comptable, au responsable de la sécurité, aux pompiers, à l'architecte, aux artisans qui travaillent sur notre chantier, peut faire de ces interlocuteurs incontournables des alliés de notre travail, et les rendre bien plus réceptifs à nos demandes forcément saugrenues. Plutôt que de simplement l'exposer dans des vitrines, il faut avant tout savoir raconter son patrimoine et le mettre en scène : quelle que soit la distance qui nous sépare des contenus,

9. La norme AFNOR NF Z 40-011, Méthode d'évaluation de l'état physique des fonds d'archives et de bibliothèques, propose une méthode très simple, utilisable aussi bien pour des sondages que pour un examen systématique des documents. 
tout le monde peut se montrer sensible à l'émotion que suscite le contact physique d'un livre vieux de plusieurs siècles, fabriqué entièrement à la main, dont on vous laisse, avec une solennité voulue, tourner les pages comme un rare privilège.

\section{Et maintenant ?}

Le programme de numérisation de Cujas se poursuit toujours, en partenariat étroit avec la BnF. Les constats d'état réalisés dans la précipitation avant le déménagement sont progressivement saisis sous tableur, de manière à mettre en place une politique de conservation raisonnée. Dès maintenant, les grands formats, dépoussiérés, consolidés et rangés dans des boîtes sur mesures, sont mieux protégés des dégradations. Les archives et manuscrits sont peu à peu catalogués, en attendant leur reconditionnement. Les thèses allemandes sont en train de sortir de leur long sommeil. La décision a été prise de surseoir provisoirement aux acquisitions en antiquariat, de manière à prendre le temps de vraiment connaître les points forts du fonds, ses lacunes, et de définir une vraie politique documentaire. Quelques acquisitions exceptionnelles sont effectuées ponctuellement, la priorité étant donnée, pour l'instant, au rachat de volumes disparus. La bibliothèque reçoit régulièrement des propositions de dons, qu'elle évalue au cas par cas, mais pour l'instant, la priorité est donnée au traitement des dons anciens, en souffrance depuis des années. Il reste à mener un gros travail de correction des notices issues de la conversion rétrospective, à envisager la mise en place d'un service de numérisation à la demande, et à poser enfin les premiers jalons d'un indispensable plan d'urgence.

Chaque jour, des étudiants et des chercheurs confirmés fréquentent la salle du Patrimoine, et de nouveaux projets de coopération nous sont proposés régulièrement. Cela valait bien dix années de conversion rétrospective, un été entier de corrections d'OCR, 7357 constats d'état réalisés en six semaines, 1000 heures de dépoussiérage et de conditionnement, 92 jours/homme d'emballage, deux années de travaux, une réunion de chantier par semaine, une plongée en apnée dans le catalogue, et même la perspective de 7000 thèses en allemand gothique à cataloguer... 


\section{3}

INSCRIRE SON PROJET DANS par Thierry Claerr LES POLITIQUES PATRIMONIALES NATIONALES ET RÉGIONALES

Depuis la publication du rapport Le Patrimoine des bibliothèques de Louis Desgraves en 1982, l'État, les collectivités territoriales et les professionnels du livre ont souhaité inscrire l'écrit dans les politiques patrimoniales nationales, régionales et locales. Cette démarche a rencontré l'intérêt du public et a permis d'affirmer la nécessité des mesures de sauvegarde, de restauration et de valorisation de l'écrit ; les objectifs fixés, notamment en matière de signalement des collections, demeurent néanmoins loin d'être atteints tandis que de nouveaux défis liés aux nouvelles technologies (collections audiovisuelles, informatisation, numérisation et internet) se posent désormais aux bibliothèques. Engagé en avril 2004, le Plan d'action pour le patrimoine écrit (PAPE) mis en œuvre par la Direction du livre et de la lecture a pour objectif de promouvoir l'ensemble du patrimoine conservé dans les bibliothèques publiques.

Ce contexte favorable et ces différents niveaux territoriaux doivent être pris en compte par tout projet patrimonial afin de bénéficier de tous les accompagnements et de toutes les aides aujourd'hui disponibles.

\section{LES POLITIQUES PATRIMONIALES AU NIVEAU NATIONAL}

\section{LE MINISTĖRE DE LA CULTURE ET DE LA COMMUNICATION}

Dans un contexte décentralisé où la très grande majorité des bibliothèques publiques (bibliothèques municipales, bibliothèques départementales de prêt) relèvent des collectivités territoriales, le ministère de la Culture et de la Communication exerce les principales compétences de l'État en matière de conservation et de valorisation du patrimoine écrit : définition du cadre législatif et réglementaire, évaluation statistique de l'activité des bibliothèques publiques, contrôle technique prévu par le Code du patrimoine, soutien technique et financier à travers différents dispositifs. Pour mettre en œuvre sa politique, il s'appuie fortement sur les DRAC et sur la BnF. 
En matière de patrimoine écrit, l'objectif de l'État est d'améliorer et de moderniser les conditions de conservation et de valorisation des collections dont l'État est propriétaire, d'une part celles de la BnF, d'autre part celles provenant des confiscations révolutionnaires et consécutives à la loi de 1905 de séparation de l'Église et de l'État, conservées dans plus de 500 bibliothèques relevant de collectivités territoriales, dont les 54 bibliothèques municipales classées.

Suite à la réorganisation de l'administration centrale du ministère intervenue ces dernières années, le Service du livre et de la lecture (SLL) est rattaché en 2010 à la Direction générale des médias et des industries culturelles et comprend depuis mars 2012 un département des bibliothèques composé de deux bureaux : le Bureau de la lecture publique et le Bureau du patrimoine qui est plus spécifiquement chargé de toutes les questions concernant le patrimoine des bibliothèques placées sous la tutelle du ministère de la Culture et de la Communication. Chargé d'animer le CTR, il est amené à conseiller les bibliothécaires, notamment en matière d'acquisitions patrimoniales : il dépouille les catalogues de vente et de libraires et signale aux bibliothèques les documents pouvant les intéresser. Il peut représenter les établissements lors des ventes aux enchères parisiennes, et exercer éventuellement pour eux le droit de préemption.

\section{UN CADRE D'ACTION NATIONAL : LE PLAN D'ACTION POUR LE PATRIMOINE ÉCRIT}

Depuis avril 2004, l'action du SLL est conçue autour du PAPE qui propose un cadre stratégique et opérationnel, décliné au niveau national, régional et local, pour mobiliser les collectivités territoriales et les bibliothèques en région, face à une situation difficile en matière de conservation et de signalement des fonds patrimoniaux, en particulier ceux conservés par les bibliothèques territoriales, qui sont estimés à plus de 30 millions de documents.

À travers l'Observatoire du patrimoine écrit en région (OPER, voir plus loin p. 155), créé en 2008, le PAPE s'efforce de dresser un nouvel état des lieux des fonds. De plus, il permet d'identifier des priorités : améliorer la conservation préventive, le signalement, la valorisation des collections 
patrimoniales des bibliothèques relevant des collectivités territoriales ainsi que la formation des professionnels.

\section{LE SITE PATRIMOINE ÉCRIT ${ }^{1}$ ET L' OBSERVATOIRE DU PATRIMOINE ÉCRIT EN RÉGION}

Le $1^{\text {er }}$ juillet 2008, le Service du livre et de la lecture a lancé le site «Patrimoine écrit ». Consacré au patrimoine des bibliothèques et à l'action de l'État en région, ce nouveau site Web a deux grands objectifs :

- mieux faire connaître les actions patrimoniales des bibliothèques, programmes de fond comme actions ponctuelles, au premier rang desquelles les Journées Patrimoine écrit que le SLL organise chaque année en région ;

- renseigner sur les missions et l'appui des services de l'État: cadre réglementaire, expertise technique ou aide financière.

Sont disponibles directement sur la page d'accueil une rubrique "Actualités » ainsi qu'un accès cartographique à l'OPER.

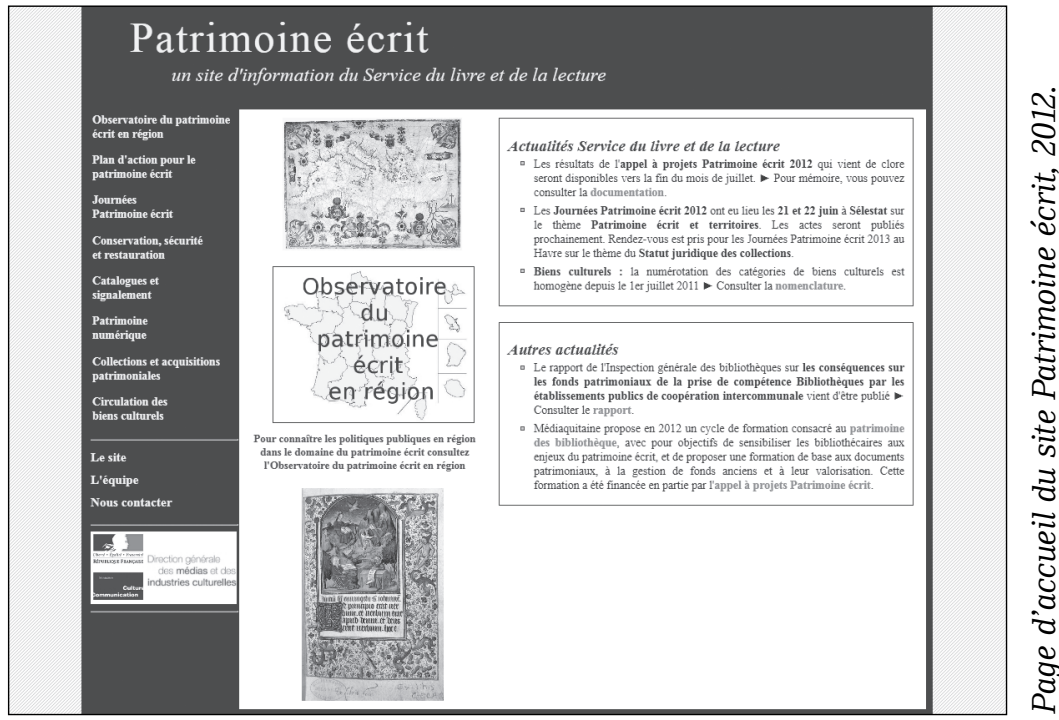

1. < http://www.patrimoineecrit.culture.gouv.fr >. 
Outil de connaissance des politiques publiques de l'État et des principales réalisations en région, l'OPER a pour mission de présenter des synthèses des politiques régionales sur le patrimoine écrit : les acteurs, les priorités, les moyens, les projets en cours, les réalisations. Ces synthèses sont élaborées en collaboration avec les conseillers pour le livre en DRAC. Elles bénéficient d'une grande variété de sources d'information que le SLL s'efforce de rassembler tout au long de l'année : programmes des services de l'État en région (DRAC), partenariats des pôles associés de la BnF, rapports de l'IGB, actions des structures régionales pour le livre, projets des bibliothèques.

L'Observatoire est conçu autour de fiches régionales organisées en dix chapitres thématiques : stratégies régionales, réseaux documentaires, signalement et numérisation, conservation et restauration, acquisitions patrimoniales aidées par l'État, formation, valorisation, structures régionales pour le livre, bibliothèques et fonds patrimoniaux, sources d'information et bibliographie.

\section{L'APPEL À PROJETS PATRIMOINE ÉCRIT}

Depuis 2007, le ministère de la Culture et de la Communication a mis en place un appel à projets Patrimoine écrit, destiné à soutenir des projets exemplaires concernant les collections patrimoniales des bibliothèques relevant des collectivités territoriales. Dispositif de cofinancement des projets patrimoniaux les plus remarquables, cet appel est organisé conjointement par le SLL et les DRAC.

Les projets d'un montant global d'au moins $10000 €$ doivent porter prioritairement sur des opérations de signalement de collections autres qu'imprimées, ou de conservation, en particulier les inventaires de fonds et de collections. Le financement de l'État peut atteindre jusqu'à $80 \%$ du coût du projet et les dépenses éligibles vont des crédits de vacation aux achats de matériels en passant par des prestations diverses.

\section{LA BNF, OPÉRATEUR NATIONAL DU PAPE}

$\mathrm{Au}$ sein de la $\mathrm{BnF}$, c'est le département de la coopération qui est spécifiquement chargé d'accompagner le PAPE, en s'appuyant sur ses pôles associés, le CCFr, la bibliothèque numérique Gallica, ainsi qu'une aide 
technique et financière aux opérations de conversion rétrospective et à la numérisation. Il organise également des stages de formation continue, ainsi que les Journées nationales des pôles associés et de la coopération, et gère la liste de diffusion poles.associes@bnf.fr.

Depuis 2006, il met en place de nouveaux " pôles régionaux » s'appuyant sur les DRAC, les structures de coopération et les bibliothèques de dépôt légal imprimeur. Plusieurs conventions ont ainsi été signées ces dernières années (Aquitaine, Basse-Normandie, Bourgogne, Champagne-Ardenne, Franche-Comté, Guadeloupe, Guyane, Haute-Normandie, LanguedocRoussillon, Limousin, Martinique, Midi-Pyrénées, Picardie, Rhône-Alpes), d'autres sont en cours de signature.

\section{LE MINISTÈre DE L'ENSEIGNEMENT SUPÉRIEUR ET DE LA RECHERCHE}

C'est la Mission de l'information scientifique et du réseau documentaire (MISTRD) qui suit plus particulièrement le patrimoine des bibliothèques universitaires et spécialisées placées sous la tutelle du ministère de l’Éducation nationale, de l'Enseignement supérieur et de la Recherche. Ses missions dans ce domaine concernent la conservation des collections, les acquisitions (suivi des préemptions en lien avec le Bureau du patrimoine du SLL) ainsi que la valorisation - essentiellement la numérisation. La MISTRD s'appuie sur des établissements nationaux placés sous sa tutelle, au premier lieu desquelles pour le patrimoine écrit :

- l'ABES chargée de la gestion et du développement du Sudoc, et de Calames ${ }^{2}$, d'outils de référencement, comme le portail national des thèses, et du signalement des fonds, y compris numérisés ;

- l'École nationale supérieure des sciences de l'information et des bibliothèques (enssib), chargée de la formation initiale et continue, qui héberge également la liste de diffusion Bibliopat et organise chaque année les Rencontres HenriJean Martin ;

2. Voir plus haut l'article de Florent Palluault, pp. 125-128. 
- le Centre technique du livre de l'enseignement supérieur (CTLes) pour les collections « d'intérêt patrimonial et scientifique » des bibliothèques universitaires d'Île-de-France relevant de l'enseignement supérieur.

\section{L'INSPECTION GÉNÉRALE DES BIBLIOTHĖQUES}

L'IGB est un service de contrôle, d'évaluation et de conseil placé sous l'autorité du ministre de l'Éducation nationale, de l'Enseignement supérieur et de la Recherche et mis à la disposition du ministre de la Culture et de la Communication pour les bibliothèques qui relèvent de sa compétence. Le suivi des régions est réparti entre les membres de l'IGB selon une liste consultable sur le site de l'IGB ${ }^{3}$ et dans l'OPER.

\section{LES POLITIQUES PATRIMONIALES AU NIVEAU RÉGIONAL}

++++++++++++++++++++++++++++++++++++++++++++++++++++++++++++++++++++++++

À l'échelon régional, la mise en réseau des bibliothèques peut jouer un rôle essentiel dans l'accompagnement des politiques patrimoniales des établissements implantés sur ce territoire.

\section{LES INTERLOCUTEURS EN RÉGION : LES CONSEILLERS LIVRE ET LECTURE AU SEIN DES DRAC}

$\mathrm{Au}$ sein des DRAC, les conseillers livre et lecture sont, avec les conservateurs d'État des bibliothèques municipales classées ou des bibliothèques universitaires conservant un fonds patrimonial important, des interlocuteurs importants pour un établissement souhaitant obtenir un conseil, une aide ou une information, en particulier pour la gestion d'un fonds patrimonial. Ils disposent de crédits déconcentrés et ont vu leur rôle renforcé par le PAPE, chaque DRAC étant amenée à structurer la dynamique régionale autour du signalement, de la conservation, de la valorisation du patrimoine écrit ou de la formation des agents, par les Centres régionaux de formation aux carrières de bibliothèques (CRFCB).

3. < http://www.enseignementsup-recherche.gouv.fr/pid24765/inspection-generale-desbibliotheques.html >. 
La dynamique de gestion et de valorisation du patrimoine écrit peut être relayée par l'agence régionale de coopération lorsqu'elle existe et qu'elle a acquis la compétence patrimoniale. Des commissions patrimoine et des catalogues collectifs peuvent alors être mis en œuvre, comme en Rhône-Alpes avec l'Agence Rhône-Alpes pour le livre et la documentation (ARALD) ou en Franche-Comté avec l'Agence comtoise de coopération pour la lecture, l'audiovisuel et la documentation (ACCOLAD).

\section{Dans le cadre du PAPE, les plans d'action régionaux pilotés par les DRAC}

À partir des orientations nationales, les conseillers livre et lecture au sein des DRAC ont développé des plans d'action régionaux, feuilles de route fixant des priorités pour chaque région (plan de numérisation concertée, de conservation partagée, opérations de formation, d'informatisation, d'inventaire, expositions, etc.) et proposant une répartition des tâches entre les acteurs locaux (les bibliothèques, parfois les services d'archives, les musées...), régionaux (les DRAC, les agences de coopération, les centres de formation, les universités...) et nationaux (BnF). Une quinzaine de régions sont dotées de tels dispositifs, aujourd'hui bien implantés.

\section{Les dispositifs État-Région : fonds régionaux d'acquisition des bibliothèques (FRAB), contrats de projet État-Région (CPER) et conventions spécifiques}

Créés par convention entre l'État et les conseils régionaux, les FRAB participent à l'acquisition de collections patrimoniales. Toutes les régions n'en sont pas dotées. Il en existe onze : Aquitaine, Auvergne, Basse-Normandie, Bretagne, Centre, Champagne-Ardenne, Haute-Normandie, Lorraine, Midi-Pyrénées, Pays-de-la-Loire, Rhône-Alpes. Certains FRAB ont élargi leurs compétences et ont ajouté le « $\mathrm{r}$ » de restauration à leur nom pour devenir des FRRAB. D'autres ont même rendu éligibles les dépenses pour la numérisation et la valorisation.

Dans une région non pourvue de FRAB, comme la Franche-Comté, le conseil régional finance néanmoins des opérations de restauration et de 
numérisation. Dans cette région comme en Lorraine, le soutien à la numérisation du patrimoine écrit est inscrit dans le CPER.

D'autres projets patrimoniaux régionaux font l'objet d'une convention spécifique entre une collectivité, la DRAC et la Région, comme le catalogue collectif de Bretagne (fonds patrimoniaux et locaux des bibliothèques de Bretagne) géré par la Bibliothèque de Rennes Métropole.

\section{QUELS SONT LES PROGRAMMES NATIONAUX DE SIGNALEMENT ?}

La mise en place en 2001 du CCFr a permis de réunir sous un portail commun les catalogues d'imprimés de la $\mathrm{BnF}$, des bibliothèques universitaires (Sudoc) et ceux des fonds anciens ou spécialisés de bibliothèques municipales (Base Patrimoine) : en tout plus de 30 millions de documents recensés. L'équipe du CCFr tient également à jour le Répertoire national des bibliothèques et des fonds documentaires (RNBFD) qui recense toutes les bibliothèques et décrit les principaux fonds qui y sont conservés.

Dans le cadre du CCFr, à côté de l'accompagnement de nombreuses opérations d'informatisation rétrospective (ou rétroconversion) des catalogues des bibliothèques en région, sont menés de vastes chantiers bibliographiques et scientifiques, comme l'actualisation du Catalogue général des manuscrits (CGM) auquel a été joint le Répertoire des manuscrits littéraires français $d u X^{e}$ siècle (PALME), l'informatisation du Patrimoine musical en région (PMR) et de la Bibliographie de la presse française politique et d'information générale (BIPFPIG). D’autres projets sont en cours, comme celui de l'informatisation des Catalogues régionaux des incunables. Malgré ces efforts, de nombreuses collections sont encore mal signalées : dans certaines régions, plus de $40 \%$ des fonds ne sont pas catalogués, voire inventoriés. 


\section{ENCADRÉ 1}

\section{DISPOSITIFS SPÉCIFIQUES DE FINANCEMENTS DU SIGNALEMENT DES COLLECTIONS}

\section{Pour les institutions relevant du ministère de l'Enseignement supérieur et de la Recherche}

La conversion rétrospective des catalogues des bibliothèques universitaires a bénéficié d'un programme continu de la part du ministère de l'Enseignement supérieur et de la Recherche, pour la période 2000 à 2010, sous la forme de deux marchés nationaux (Sudoc) et, au sein des contrats d'établissement, de financements pour des opérations de conversion rétrospective spécifiques ou ponctuelles (Sudoc ou Calames). Par ailleurs, sur la même période (2000-2010), les bibliothèques membres du réseau Sudoc ont mené des chantiers d'informatisation rétrospective (ou rétroconversion) sur leur propre budget.

À partir de 2010, la mission « rétroconversion » a été transférée du ministère
(ex-SDBIS) à l'ABES :

- l'ABES est chargée d'élaborer une politique de signalement rétrospectif, en définissant des priorités pour le Sudoc et pour Calames ;

- l'ABES apporte une aide financière directe aux établissements dont les projets de rétroconversion ont été retenus dans le cadre des appels à projets.

Par ailleurs, les établissements d'enseignement supérieur demeurent libres de financer sur leur propre budget toute opération de conversion rétrospective qui leur sera nécessaire dans le catalogue Sudoc ou dans Calames.

Pour en savoir plus : < http://www.abes. $\mathrm{fr} / S u d o c / S e r v i c e s-d i s p o n i b l e s-a u t o u r-$ $\mathrm{du}$-Sudoc/Retroconversions > $<$ http://www.abes.fr/Calames/Servicesdisponibles-pour-Calames/Retroconversions >

\section{Pour les institutions relevant du ministère de la culture et de la communication}

- La politique de coopération de la BnF pour des opérations de rétroconversion des fonds anciens et des fonds locaux et spécialisés (sans limite de date) dans le cadre d'une convention de pôle associé ; Financement : de $50 \%$ à $70 \%$ (financement à $100 \%$ pour les extractions).

Type d'opération financée : marché de catalogage privilégié au financement de vacations.
Contact : service CCFr du département de la coopération de la BnF.

Dans certaines régions (Haute-Normandie, Picardie...), des programmes spécifiques sont inscrits dans la dynamique des Pôles associés régionaux.

- L'appel à projets " Patrimoine écrit " du SLL pour des opérations de signalement de collections autres qu'imprimées ou pour des inventaires de fonds et de collections. 


\section{QUELS SONT LES PROGRAMMES NATIONAUX DE NUMÉRISATION?}

La reproduction des collections qui assure leur conservation et la communication sans risque des originaux, facilite leur valorisation et bénéficie de l'évolution des techniques de numérisation et des supports multimédias et interactifs. La numérisation se développe en France avec près de 500 opérations de numérisation recensées dans plus de 150 bibliothèques, avec l'enrichissement de Gallica ${ }^{4}$ et avec la réalisation de programmes nationaux, comme celui mené en partenariat avec l'IRHT, laboratoire du CNRS spécialisé dans la reproduction et l'étude des manuscrits médiévaux ${ }^{5}$.

\section{LE PANORAMA DE LA NUMÉRISATION EN BIBLIOTHĖQUE}

Mis en œuvre depuis 2001 par le ministère de la Culture et de la Communication, le catalogue des fonds culturels numérisés Patrimoine numérique ${ }^{6}$ recense les opérations de numérisation du patrimoine culturel en France et participe au programme Michael qui a pour objectif de signaler les projets au niveau européen, en fédérant les catalogues nationaux. Réalisé en concertation avec Patrimoine numérique, le catalogue Numes ${ }^{7}$ géré par l'ABES, signale les fonds numérisés par les institutions françaises de l'enseignement supérieur et de la recherche.

\section{QUELQUES SITES UTILES EN MATIÈRE DE NUMÉRISATION}

Plusieurs sites institutionnels fournissent des recommandations en matière de numérisation, des modèles de cahiers des charges et des normes techniques pour les différents supports à numériser, que ce soit le site « Numérisation du patrimoine culturel » du ministère de la Culture et de la Communication $^{8}$, le site des Archives de France ${ }^{9}$ ou les sites de la $\mathrm{BnF}^{10}$.

\footnotetext{
4. < http://gallica.bnf.fr >.

5. < http://www.irht.cnrs.fr >.

6. < http://www.numerique.culture.fr >.

7. < http://www.numes.fr >.

8. < http://www.culture.gouv.fr/culture/mrt/numerisation/index.html >.

9. < http://www.archivesdefrance.culture.gouv.fr >, rubrique e-archives.

10. < http://www.bnf.fr >, rubrique Professionnels, numérisation ; < http://bibnum.bnf.fr >.
} 


\section{LE PLAN NATIONAL DE NUMÉRISATION DU MINISTĖRE DE LA CULTURE ET DE LA COMMUNICATION}

Le ministère de la Culture et de la Communication encourage depuis 1996 la numérisation des collections patrimoniales conservées en bibliothèques, dans le cadre d'un plan national de numérisation géré par le Département de la recherche, de l'enseignement supérieur et de la technologie (DREST), avec deux objectifs clairement affichés :

- augmenter significativement l'offre de ressources numériques culturelles constituée d'ensembles cohérents et de taille critique ;

- promouvoir une consultation libre et ouverte des ressources numériques culturelles pour tous les internautes.

En s'appuyant sur les recommandations du Schéma numérique des bibliothèques et du rapport sur la numérisation du patrimoine écrit remis en janvier 2010 par Marc Tessier, il favorise la concertation et l'interopérabilité entre les projets de numérisation et souhaite donner davantage de visibilité aux réalisations, tout en veillant à l'utilisation des normes et des bonnes pratiques en matière de numérisation et de conservation numérique. Depuis 2005, la mise en ligne et la valorisation des fonds numérisés sont ainsi devenues obligatoires pour les projets retenus.

\section{LA POLITIQUE DE COOPÉRATION NUMÉRIQUE DE LA BHF}

Le ministère de la Culture et de la Communication soutient depuis 2007, sur financement du Centre national du livre, le développement de la bibliothèque numérique Gallica, la numérisation intensive et la diffusion par ce portail des collections d'imprimés patrimoniaux conservés par la BnF et, depuis 2011, par des bibliothèques partenaires.

À côté de l'ouverture de ses chaînes de numérisation à des bibliothèques partenaires, la $\mathrm{BnF}$ a mis en œuvre des programmes de numérisation concertée, que ce soit en sciences juridiques avec la Bibliothèque interuniversitaire Cujas ou en histoire de l'art avec la bibliothèque de l'Institut national d'histoire de l'art (INHA), par le lancement d'appels à projets. 


\section{L'ÉVOLUTION DU CONCOURS PARTICULIER POUR LES BIBLIOTHĖQUES VERS UN CONTRAT NUMÉRIQUE POUR LES BIBLIOTHĖQUES}

Le concours particulier pour les bibliothèques au sein de la dotation générale de décentralisation (DGD) qui peut financer la construction, l'aménagement et l'équipement des bibliothèques territoriales, y compris les magasins et le matériel de conservation, soutient également l'informatisation et la numérisation (production et diffusion) selon deux axes :

1) aménagement d'un atelier technique et acquisition de matériels de numérisation et de conservation numérique ;

2) actions de numérisation, signalement et diffusion des collections.

Ce dispositif permet l'échelonnement sur plusieurs exercices budgétaires d'une opération de numérisation d'ampleur. Depuis 2010, dans le cadre des propositions pour le développement de la lecture du ministère de la Culture et de la Communication, il participe également au financement du programme des « bibliothèques numériques de référence ».

Les programmes d'aide aux bibliothèques en faveur des fonds patrimoniaux se sont développés dans le cadre du PAPE. À côté des outils traditionnels, sont apparus de nouveaux programmes pour susciter des dynamiques auprès des institutions patrimoniales (Plan national de numérisation, appel à projets Patrimoine écrit, pôles associés régionaux, évolution des FRAB en fonds régional pour le patrimoine) et rendre accessibles leurs fonds aux chercheurs et aux lecteurs. Dans la construction de son projet, il est important de pouvoir s'appuyer sur ces différents dispositifs d'accompagnement politique, financier et technique. 


\section{ENCADRÉ 2 DISPOSITIFS SPÉCIFIQUES DE FINANCEMENTS DE LA NUMÉRISATION}

\section{Pour les institutions relevant du ministère de l'enseignement supérieur et de la Recherche}

La politique contractuelle est le principal outil de financement de la numérisation :

- opérations de numérisation des bibliothèques universitaires via les contrats des établissements ;

- programmes de constitution de corpus scientifiques via les contrats des organismes de recherche.

Le financement au titre des très grandes infrastructures de recherche et par les appels à projets de l'Agence nationale de la recherche (ANR).

Les programmes de numérisation concertée mis en place par le département de la coopération de la BnF.

\section{Aides :}

- les contrats : passage au nouveau modèle d'allocation des moyens depuis janvier 2009 ;

- les conventions de pôles associés de la $\mathrm{BnF}$;

- la Bibliothèque scientifique numérique (BSN) et ANR-Corpus ;

- l'appel aux opérateurs (Persée, Centre informatique national de l'enseignement supérieur-CINES, Agence bibliographique de l'enseignement supérieurABES, CNRS-TGE Adonis).

\section{Pour les institutions relevant du ministère de la culture et de la communication}

- Le plan national de numérisation du ministère

Fonctionnement par appel à projets

Objectif : numériser les contenus culturels pour les diffuser gratuitement

Financement : jusqu'à $50 \%$

Projets d'une durée d'un an maximum ; définition de six programmes thématiques ; priorité aux partenariats régionaux.

\section{Pour les bibliothèques des} collectivités territoriales

- les financements locaux : municipalité, intercommunalité, conseil général, région...;

- les contrats de projet État-Région (CPER) dans certaines régions ;

- certains FRAB (ex. Basse-Normandie et Midi-Pyrénées) ;

- les crédits déconcentrés en région auprès de la DRAC ;

- les programmes de numérisation concertée de la BnF.

Financement spécifique aux bibliothèques des collectivités territoriales : le concours particulier pour les bibliothèques au sein de la DGD et programme des bibliothèques numériques de référence.

Contact : conseiller livre et lecture à la DRAC 


\section{MÉMENTO}

par Dominique Coq

\section{COMPRENDRE LA COLLECTION}

- Votre collection ne s'est pas constituée au hasard : elle a son histoire, celle des fonds qui la constituent, des libéralités (dons, legs...) qui l'ont éventuellement enrichie.

- Il faut connaître son statut, la réglementation qui la régit. Sa compréhension passe par la connaissance des choix de vos prédécesseurs, de leur "politique documentaire ».

- Le patrimoine doit être choisi, non subi, et ce choix s'opère dans la concertation.

- Une collection n'est pas figée (sauf celles qui n'ont pas vocation à s'accroître), elle a évolué avant vous et elle continuera après vous. Vous avez le devoir de la transmettre, éventuellement de l'enrichir selon des critères dont vous devrez rendre compte et en vous entourant de compétences extérieures à la bibliothèque. Car il vaut mieux bien conserver une collection en l'état que l'augmenter n'importe comment ou pour se faire plaisir.

\section{PRENDRE DU TEMPS POUR CONNAÎTRE SA COLLECTION}

$++++++++++++++++++++++++++++++++++++++++++++++++++++++++++++++++++$

Lorsqu'on est confronté à une collection qu'on ne connaît pas, il faut d'abord la faire sienne (intellectuellement, s'entend !). Cela demande du temps, même pour les professionnels familiers des questions patrimoniales. Beaucoup de temps à passer dans les magasins, beaucoup de temps à discuter avec les chercheurs, avec les collègues responsables d'autres collections patrimoniales. Cette familiarité avec la collection et les éléments qui la composent s'acquiert par une sorte de lente percolation. Rien d'étonnant : les documents, de nature variée, qui constituent votre collection ne sont pas spécialement simples à « décoder » et il faut accepter de passer du temps à leur contact pour en percer les mystères (la façon dont 
ils sont fabriqués, les informations qu'ils contiennent, les manières de les décrire, les instruments de travail à utiliser, imprimés ou en ligne). Il n'y a pas que des documents précieux dans une collection patrimoniale. Ce n'est pas une raison pour négliger les pièces les plus modestes et ne décrire que les plus spectaculaires. Tous les documents doivent être décrits sans exclusive. Votre collection n'est pas seule au monde et s'inscrit dans un paysage documentaire plus général. Ainsi tel document qui peut paraître mineur, pris isolément, prend sens avec d'autres conservés ailleurs, dans une perspective sérielle qui peut s'avérer très riche pour l'historien. Et même d'un point de vue plus local, les informations particulières (provenances, mentions manuscrites) que contient éventuellement un exemplaire d'une édition quelconque peuvent se révéler parfois d'un grand intérêt.

\section{PROTÉGER LA COLLECTION}

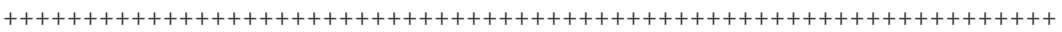

Bien conserver - ou plutôt préserver dans les meilleures conditions - sa collection afin de la transmettre la moins dégradée possible aux générations suivantes. Cela peut paraître simpliste ou secondaire comme mission. Ce ne l'est pas. Tout un ensemble de savoirs, de savoir-faire est à acquérir pour y parvenir et à faire comprendre à vos collègues, aux utilisateurs, aux lecteurs, à votre tutelle. Mais entre le désir de communiquer le plus possible, de multiplier les expositions et la nécessité de restreindre l'accès au document pour le préserver de la lumière ou des manipulations parfois hasardeuses des lecteurs, où placer le curseur ? Contrairement à ce que l'on croit, les livres les plus anciens ne sont pas les plus fragiles, et on a souvent la surprise de constater en les ouvrant leur état de fraîcheur alors qu'ils ont traversé les siècles dans des conditions de conservation qui étaient loin d'être les meilleures. Au reste, tout n'est pas à protéger avec la même inquiète sollicitude. Repérer les éléments les plus précieux et les plus fragiles à sauvegarder spécialement est une des premières tâches du responsable patrimonial pour établir un plan d'urgence en cas de sinistre. 


\section{FAIRE CONNAÎTRE LA COLLECTION}

C'est le point essentiel. S'approprier, comprendre sa collection, bien la conserver, c'est parfait, mais le plus important est de la faire connaître. On estime à plus de $40 \%$ des collections patrimoniales qui ne sont pas signalées ${ }^{1}$. C'est-à-dire qu'elles sont là, prennent de la place mais n'existent pour personne : une sorte d'équivalent de la matière noire pour les astrophysiciens.

Signaler, encore signaler, toujours signaler : avant de songer à faire des acquisitions ou à organiser des expositions, avant d'entreprendre des opérations de numérisation ou de valorisation, il faut d'abord faire savoir ce qui est conservé sur les rayons et le décrire afin de le verser dans les réservoirs bibliographiques les plus larges et les plus consultés. Cela passe par quelques opérations indispensables, qui peuvent d'ailleurs bénéficier d'un soutien financier et d'une expertise scientifique de la part des acteurs nationaux et régionaux de signalement :

- signalement des différents fonds tout d'abord dans le RNBFD du CCFr ;

- informatisation des anciens catalogues (rétroconversion) afin d'en verser les notices dans le CCFr ou le Sudoc.

Le signalement des documents patrimoniaux qui n'ont pas encore été catalogués met en jeu des connaissances spécifiques qu'il convient d'acquérir dans des stages de formation si on ne les possède pas encore :

- pour décrire les différents types de document (manuscrit, gravure, photo, etc.) ;

- pour relever les particularités de l'exemplaire considéré (histoire, anciens possesseurs, reliure, rareté, etc.) ;

- pour apprendre et utiliser le format bibliographique recommandé selon le type de document.

1. Plan d'action pour le patrimoine écrit et graphique, Synthèse nationale des enquêtes régionales, 2007, [en ligne] : < http://www.patrimoineecrit.culture.gouv.fr/PAPE.html >, p. 10. 


\section{TROUVER DES PARTENAIRES POUR UN CADRE D'ACTION}

Votre travail ne sera utile que s'il se fait en concertation et coopération, à l'intérieur de la bibliothèque et à l'extérieur de l'établissement. Des partenariats scientifiques et techniques sont à chercher au niveau local (autres établissements de conservation, universités, sociétés savantes), au niveau régional (DRAC, structures régionales de coopération), au niveau national (BnF, SLL) :

- pour les choix documentaires qui devront être opérés pour la constitution de votre collection patrimoniale et éventuellement pour la constitution d'une réserve en son sein ;

- pour déterminer les projets à mettre en œuvre pour exploiter et valoriser cette collection (appels à projets, coopération avec la BnF).

\section{À VOUS DE JOUER MAINTENANT}

Vous savez désormais comment vous situer vis-à-vis de votre fonds : il ne s'agit pas pour vous de devenir un spécialiste du livre ancien, de la gravure, ou de la reliure mais, d'une part, de vous débrouiller suffisamment avec ce type de document pour les faire connaître à ceux qui sont le plus à même de les exploiter et, d'autre part, de faire bénéficier des richesses de votre collection patrimoniale au plus grand nombre. Les pages qui précèdent ont tenté de vous expliquer quelques règles de base.

D'ailleurs, sachez que vous n'êtes pas seul-e. Des ressources existent en ligne. D'abord la liste de discussion BiblioPat ${ }^{2}$ qui regroupe de nombreux responsables de fonds patrimoniaux auxquels vous pourrez avoir recours en cas de besoin et qui répondront à vos interrogations même si elles vous paraissent ingénues. Et n'oubliez pas de mettre dans vos favoris l'URL du site Patrimoine écrit ${ }^{3}$ du SLL et celui des fiches destinées aux professionnels sur le site de la $\mathrm{BnF}^{4}$. Vous y trouverez les réponses à beaucoup de questions que vous pourrez vous poser.

2. < http://listes.enssib.fr/listes/info/bibliopat >.

3. < http://www.patrimoineecrit.culture.gouv.fr/index.html >.

4. < http://www.bnf.fr/fr/professionnels.html >. 


\section{SIGLES ET ACRONYMES}

ANR

Agence nationale de la recherche

BIPFPIG

Bibliographie de la presse française politique et d'information générale

$\mathrm{BnF}$

Bibliothèque nationale de France

BSN

Bibliothèque scientifique numérique

\section{Calames}

Catalogue en ligne des archives et des manuscrits de l'enseignement supérieur

\section{$\mathrm{CCFr}$}

Catalogue collectif de France

CGM

Catalogue général des manuscrits des bibliothèques publiques de France

\section{CPER}

Contrat de projet État-Région

CRCC

Centre de recherche et de conservation des collections, laboratoire du CNRS à Paris

\section{CRFCB}

Centre régional de formation aux carrières de bibliothèques

\section{CTLes}

Centre technique du livre de

l'enseignement supérieur

\section{CTR}

Comité technique restauration

DGD

Dotation générale de décentralisation

\section{DRAC}

Direction régionale des affaires culturelles

\section{DREST}

Département de la recherche, de l'enseignement supérieur et de la technologie au secrétariat général du ministère de la Culture et de la Communication

DTD

Document Type Definition (Définition de type de document)

\section{EAD}

Encoded Archival Description

(Description archivistique encodée)

\section{FRBR}

Functional Requirements of Bibliographic Records (fonctionnalités requises des notices bibliographiques)

\section{FR(R)AB}

Fonds régional d'acquisition (et de restauration) des bibliothèques 
HR

Humidité relative

INHA

Institut national de l'histoire de l'art

IRHT

Institut de recherche et d'histoire des textes, laboratoire du CNRS à Paris et Orléans

ISBD

International Standard Bibliographic Description

MARC

MAchine-Readable Cataloging

MISTRD

Mission de l'information scientifique et du réseau documentaire (ministère de l'Enseignement supérieur et de la Recherche)

\section{OCR}

Optical Character Recognition (reconnaissance optique de caractères)

\section{OPER}

Observatoire du patrimoine écrit en région, sur le site Patrimoine écrit du Service du livre et de la lecture

\section{PALME}

Répertoire des manuscrits littéraires français du $\mathrm{xx}^{\mathrm{e}}$ siècle, accessible via le CCFr

\section{PAPE}

Plan d'action pour le patrimoine écrit et graphique

\section{PMR}

Patrimoine musical en région

\section{RFID}

Radio Frequency IDentification

(Identification par radio fréquence)

\section{RISM}

Répertoire international des sources musicales

\section{RNBFD}

Répertoire national des bibliothèques et des fonds documentaires

\section{SLL}

Service du livre et de la lecture à la Direction générale des médias et des industries culturelles (ministère de la Culture et de la Communication)

\section{SUD0C}

Système universitaire de documentation

\section{TEI}

Text Encoding Initiative

\section{XML}

Extensible Markup Language (langage de balisage étendu) 


\section{GLOSSAIRE}

Anoxie

Traitement de désinfection d'insectes par absorption de dyoxygène sous atmosphère d'azote.

\section{Bandeau}

Ornement rectangulaire situé en tête de la page d'un livre. Au début gravé en bois, il se présente ensuite sous forme de vignette en fonte ou en taille-douce.

\section{Banderolage}

Opération de conditionnement visant au maintien de parties détachées d'un volume relié (dos, plat...) à l'aide de bandes de papier neutre.

\section{Bifeuillet}

Pièce rectangulaire de parchemin ou de papier pliée en son milieu pour former deux feuillets. C'est l'élément de base du cahier.

\section{Bloc de cahiers}

Ensemble des cahiers formant un volume, assemblés dans l'ordre. On dit aussi « corps du volume » ou « bloctexte ».

\section{Boulon ou bouillon}

Clou de métal à grosse tête fixé sur le plat du volume et destiné à éviter l'usure du plat par frottement sur le pupitre.

\section{Cahier}

Ensemble de bifeuillets emboîtés les uns dans les autres et cousus par leur pli. C'est l'élément de base du livre qui est un ensemble de cahiers.

\section{Chant ou champ (d'une reliure)}

Épaisseur du plat.

\section{Coiffe}

Repli ou bourrelet formé par la couvrure, en tête et en queue du dos, pour protéger la tranchefile.

\section{Collection}

Ensemble ordonné de documents, ou de documents et d'objets.

\section{Comité technique restauration (CTR)} Instance d'expertise et de conseil fournissant des éléments d'appréciation au préfet de région chargé d'émettre un avis, dans le cadre du Contrôle technique de l'État, sur les projets de restauration élaborés par les collectivités territoriales pour les collections dont elles ont la charge. Placé auprès du Service du livre et de la lecture au ministère de la Culture et de la Communication, le CTR est composé de 12 membres (conservateurs, restaurateurs, historiens du livre). 


\section{Conditionnement}

Mode de protection des documents permettant de les isoler des agressions externes.

\section{Contrôle climatique}

Prise en compte des conditions climatiques (chaleur, humidité, isolation, ventilation, filtrage de l'air, etc.) pour la conservation des documents.

\section{Contrôle technique de l'État}

Ensemble de dispositions réglementaires s'appliquant aux collections conservées dans les bibliothèques de collectivités territoriales ou d'établissements publics de coopération intercommunale (Code du patrimoine, articles R. 310-2 à R. 310-13).

\section{Couvrure}

Garnissage des plats et du dos du volume d'un matériau souple tel que peau, étoffe...

\section{Cul-de-lampe}

Ornement typographique, souvent de forme triangulaire, placé au bas d'un chapitre.

\section{Cuvette}

Empreinte laissée par l'épaisseur de la plaque gravée en métal dans le papier d'une estampe en taille-douce.

\section{Dation}

Offre à l'État d'une œuvre d'art ou d'un document patrimonial majeur en échange de certaines obligations fiscales

\section{Défet}

Feuillet dépareillé d'un livre qui peut servir à compléter un exemplaire défectueux. Les défets sont souvent utilisés par le relieur pour constituer les plats ou renforcer le dos.

\section{Dépoussiérage}

Opération de nettoyage des magasins et des documents, visant à les débarrasser des particules ténues qui s'y déposent et s'y insèrent.

\section{Dermeste}

Un des insectes destructeurs des documents de bibliothèque en $\mathrm{y}$ creusant des galeries sinueuses.

\section{Désinfection}

Traitement des documents et des locaux contre les champignons, insectes et bactéries.

\section{Domanialité publique}

Régime juridique des biens composant le domaine public.

\section{Dublin Core}

Ensemble de recommandations pour la description bibliographique minimale de documents ou objets sur le Web.

\section{Encarter}

Insérer dans un cahier.

\section{Ex-dono}

Formule notifiant le don d'un objet ou d'un livre par une personne (morale ou physique) à une autre. 


\section{Ex-libris}

Formule manuscrite ou étiquette gravée notifiant l'appartenance d'un livre à une personne physique ou morale.

\section{Feuillet}

Chacune des deux moitiés d'un bifeuillet. Chacune des deux faces d'un feuillet s'appelle une page.

\section{Filtre absolu}

Dispositif permettant une efficacité maximale des aspirateurs, en évitant toute redispersion des poussières les plus fines.

\section{Fleuron}

Ornement typographique à motif floral, ornant le titre ou les blancs du texte.

\section{Fonds}

Collection de documents, ou de documents et d'objets, considérés du point de vue de leur unité d'intérêt, d'histoire ou de provenance.

\section{Futon modulable de soutien}

Rectangle de tissu rembourré de laine de polyester (type dacron) et maintenu enroulé grâce à un système d'attache par Velcro, formant ainsi un support modulable pour la consultation des volumes fragiles.

\section{Humidité relative}

Rapport entre la quantité de vapeur d'eau dans un volume d'air par la capacité d'absorption de l'air à la même température.

\section{Hygrométrie}

Teneur en eau de l'air environnant.

\section{Incunables}

Premiers livres imprimés, depuis l'invention de Gutenberg (milieu $x V^{\mathrm{e}}$ siècle) jusqu'à la date purement conventionnelle du 31 décembre 1500.

\section{Lépisme}

Insecte polyphage (appelé aussi poisson d'argent) contribuant à la dégradation des documents de bibliothèque en creusant des galeries sinueuses.

\section{Lettrine}

Lettre initiale majuscule décorée placée en tête d'un texte ou d'un chapitre et occupant une hauteur supérieure à la ligne courante. En usage dans les manuscrits, elles ont été très tôt utilisées dans la typographie. On dit aussi « lettre ornée ».

\section{Lux}

Quantité de lumière reçue par une surface donnée.

\section{Mors}

Partie de la couvrure qui fait charnière entre le dos et les plats d'une reliure.

\section{Neutre (matériau)}

Sans acide. 


\section{Océriser}

Transformer automatiquement un fichier contenant l'image d'un document en fichier texte, grâce à un logiciel de reconnaissance optique de caractères (OCR).

\section{Plan d'urgence}

Plan d'intervention concerté à

l'avance visant à prévenir tout risque pour les collections patrimoniales et à minimiser le sinistre s'il intervient quand même.

\section{Plan d'action pour le patrimoine} écrit

Mis en œuvre depuis 2004 par la Direction du livre et de la lecture pour inventorier et promouvoir l'ensemble du patrimoine conservé dans les bibliothèques publiques.

\section{Point de rosée}

Point où l'humidité relative est égale à $100 \%$ à une température donnée.

\section{RFID}

Système d'identification de documents comprenant une étiquette électronique pour mémoriser des informations et un lecteur.

Le transfert d'information $\mathrm{du}$ composant électronique vers le lecteur s'effectue par radiofréquence.

\section{Taille-douce}

Procédé de gravure en creux (burin, eau-forte, pointe sèche, etc.)

\section{Thermo-hygromètre}

Matériel électronique mesurant à la fois la température (généralement en ${ }^{\circ} \mathrm{C}$ ) et l'humidité (en \% du seuil de saturation).

\section{Tranchefile}

Élément qui renforce ou soutient les deux extrémités du dos, au niveau des coiffes.

\section{Vignette}

Motif ornemental souvent xylographié, illustrant les pages de textes imprimés, notamment la page de titre.

\section{Vrillette}

Insecte xylophage parmi les plus redoutables agents de destruction $\mathrm{du}$ papier (petits trous ronds). 


\section{BIBLIOGRAPHIE}

\section{LE PATRIMOINE. GÉNÉRALITÉS}

Louis Desgraves et Jean-Luc

Gautier, Le patrimoine des

bibliothèques : rapport à Monsieur le Directeur du Livre et de la Lecture. Paris, ministère de la Culture, 1982.

Pascal Fouché (dir.), Dictionnaire encyclopédique du livre. 3 volumes + 1 index. Paris, Éditions du Cercle de la Librairie, 2002-2011.

Histoire des bibliothèques françaises, 4 vol. Paris, Promodis-Éditions du Cercle de la Librairie, 1988-1992.

Raphaële Mouren (dir.), Manuel du patrimoine en bibliothèque. Paris, Éditions du Cercle de la Librairie, 2007.

Patrimoine des bibliothèques de France : un guide des régions. 10 volumes +1 index. Paris, Banques CIC pour le livre ; ministère de la Culture et de la Communication; Payot, 1995.

Patrimoine écrit, Patrimoine vivant. Règles élémentaires pour la conservation et la valorisation des documents patrimoniaux. [Paris,] FFCB, 1999.
Hélène Richard, La formation aux questions patrimoniales dans les bibliothèques : rapport à $M$. le ministre de la Culture et de la Communication, Inspection générale des bibliothèques, septembre $2010\left(\mathrm{n}^{\circ} 2010\right.$ 016). [En ligne] : < http://media. enseignementsup-recherche.gouv.fr/ file/2010/52/1/Formation_aux_ questions_patrim_def_166521.pdf >.

\section{CONSERVATION ET RESTAURATION}

$++++++++++++++++++++++++++++$

Claude Adam, Restauration des manuscrits et des livres anciens. Puteaux, Erec, 1984.

Jean-Marie Arnoult (dir.), Protection et mise en valeur du patrimoine des bibliothèques : recommandations techniques. Paris, Direction du livre et de la lecture, 1998. [En ligne] : < http:// www.patrimoineecrit.culture.gouv.fr/ ConsRest/conservation_dll.html >.

Bibliothèque nationale de France, Fiches pratiques : la conservation préventive. [En ligne]:

< http://www.bnf.fr/fr/professionnels/

conservation_fiches_pratiques/ s.conservation_fiches_prevention. html >. 


\section{IDENTIFIER ET SIGNALER LES DOCUMENTS}

André Béguin, Dictionnaire technique de l'estampe. Nouv. éd. augmentée. Paris, l'auteur, 1998. Voir son « Petit dictionnaire technique de l'estampe ». [En ligne] : < http://www.presseestampe.com/index.php?page $=$ dico $>$.

Anne Cartier-Bresson (dir.), Le vocabulaire technique de la photographie. Paris, Marval, Paris-Musée, 2008.

Michel Frizot (dir.), Nouvelle histoire de la photographie, Paris, LarousseAdam Biro, 2001.

Alìs Krejèa, Les techniques de la gravure. Guide des techniques et de l'histoire de la gravure d'art originale. Trad. Fr. Paris, Gründ, 1983.

Bertrand Lavédrine, (re)Connaître et conserver les photographies anciennes. Paris, Éd. du CTHS, 2007.

Denis Muzerelle, Vocabulaire codicologique, Répertoire méthodique des termes français relatifs aux manuscrits. Paris, Ed. CEMI, 1985. Une version adaptée au Web est disponible en ligne : < http://vocabulaire.irht. cnrs.fr/ $>$.
Jean Toulet, Introduction à l'histoire de la reliure française, $X V^{e}-X V I I I^{e}$ siècles, Paris, Bibliothèque nationale, 1973. [En ligne] : < http://www. patrimoineecrit.culture.gouv.fr/Cata/ toulet_reliure.pdf >.

Dominique Varry, Introduction à la bibliographie matérielle. Archéologie du livre imprimé (1454 - vers 1830). Ouvrage évolutif mis en ligne pour la première fois le 15 juin 2011 : < http:// dominique-varry.enssib.fr/bibliographie\%20materielle $>$.

\section{LISTE DE DIFFUSION}

$++++++++++++++++++++++++++++$

Liste Patrimoine-bibliothèques

(Service du livre et de la lecture).

Contact : patrimoine-bibliotheques@

culture.gouv.fr

\section{LISTE DE DISCUSSION}

$++++++++++++++++++++++++++++++$

Liste de l'association BiblioPat hébergée par l'enssib : < http://listes.enssib. fr/wws/info/bibliopat $>$. 


\section{LISTE DES ILLUSTRATIONS}

Encadré. Quelques bibliothèques publiques avant 1789

p. 14

Encadré. La Révolution et les bibliothèques en 7 dates p. 15

Encadré. De quoi l'État se mêle-t-il ? p. 19

Encadré. Les fonds d’État p. 21

Encadré. Les documents à mettre en réserve pp. $41-42$

Schéma. L'humidité relative p. 47

Tableau. Les conséquences de la température et de l'humidité sur les documents p. 48

Tableaux. Actions possibles pour améliorer les conditions climatiques .. p. 49

Tableau. Effets et conséquences des radiations sur les documents p. 50

Tableau. Les effets de la pollution p. 51

Tableau. Sensibilité des types de document à la lumière p. 61

Encadré. Les interventions en cas d'inondation

(et en phase post-incendie) pp. 64-65

Tableau. Les formats de base p. 72

Schéma. Pliage : les formats p. 75

Schéma. Les parties constitutives d'une reliure p. 81

Tableaux. Typologie des reliures soignées et courantes :
Mi $x v^{\mathrm{e}}-\mathrm{XVI}^{\mathrm{e}}$ siècle pp. $88-89$
$\mathrm{XVII}^{\mathrm{e}}$ siècle pp. $90-91$
XVIII ${ }^{\mathrm{e}}$ siècle pp. $92-93$
$\mathrm{XIX}^{\mathrm{e}}$ siècle p. 94 
Schéma. Techniques de l'estampe

Encadré. Données à signaler dans une notice d'estampe p. 106

Encadré. Identification et support des négatifs monochromes p. 110

Tableau. Identification des différents types de négatifs sur verre p. 112

Encadré. Principales altérations du support p. 114

Tableau. Principaux procédés photographiques p. 115

Schéma. Représentation des épreuves photographiques sur papier p. 116

Focus. Soutien financier aux opérations d'informatisation p. 119

Focus. Le XML p. 120

Focus. Le format MARC - Livre ancien p. 121

Focus. La TEI p. 123

Focus. Décrire des manuscrits et archives : ISAD(G) et DeMarch p. 124

Focus. Fonctionnalités XML p. 126

Focus. Dublin Core p. 129

Page d'accueil du site Patrimoine écrit p. 154

Encadré. Dispositifs spécifiques de financements du signalement des collections p. 160

Encadré. Dispositifs spécifiques de financements de la numérisation .. p. 164 


\section{LISTE DES AUTEURS}

Noëlle Balley

Chef du Département des

monographies,

Bibliothèque Cujas, Paris

\section{Thierry Claerr}

Adjoint au chef du Département des bibliothèques, Service du livre et de la lecture, ministère de la Culture et de la Communication

\section{Gérard Cohen}

Conservateur général au

Service du livre et de la lecture, ministère de la Culture et de la Communication

\section{Dominique Coq}

Chef du Bureau du patrimoine, Service du livre et de la lecture, ministère de la Culture et de la Communication

\section{Jocelyne Deschaux}

Responsable du service

Patrimoine écrit, Bibliothèque d'étude et du patrimoine de Toulouse

\section{Bernard Huchet}

Chef du service de la documentation normande \& patrimoine de la Bibliothèque de Caen

\section{Carole Jacquet}

Responsable des ressources

documentaires,

Centre culturel irlandais (Paris)

\section{Laure Jestaz}

Adjointe à la Directrice de la Bibliothèque historique de la Ville de Paris

\section{Patrick Lamotte}

Responsable de l'atelier de restauration au Département des estampes et de la photographie, Bibliothèque nationale de France

\section{Fabienne Le Bars}

Conservateur en chef au

Département de la réserve des livres rares, Bibliothèque nationale de France

\section{Séverine Lepape}

Responsable du service de l'estampe ancienne et de la réserve au Département des estampes et de la photographie, Bibliothèque nationale de France

\section{Florent Palluault}

Chargé de mission au Bureau du patrimoine, Service du livre et de la lecture, ministère de la Culture et de la Communication 
Secrétariat d'édition:

Silvia Ceccani

Mise en page:

Alexandre Bocquier

Conception graphique:

atelier Perluette, 69001 Lyon.

< http://www.perluette-atelier.com >

Achevé d'imprimer en octobre 2012 imprimerie forézienne

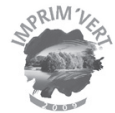

dépôt légal: $2^{\text {nd }}$ semestre 2012 


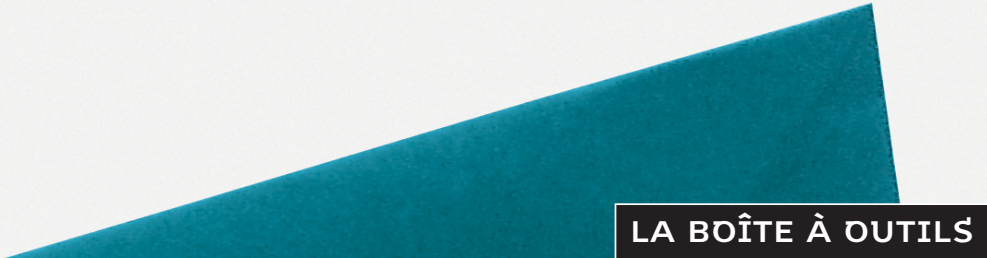

\section{APPRENDRE À GÉRER DES COLLECTIONS PATRIMONIALES EN BIBLIOTHĖQUE}

Vous venez d'être nommé-e responsable de la collection patrimoniale d'une bibliothèque mais vous n'êtes pas spécialiste ou vos connaissances sont lointaines. D'où viennent ces vieux livres qui dorment dans la réserve? Qu'en faire? A-t-on le droit de s'en débarrasser s'ils paraissent ne servir à personne? Y en a-t-il qui sont rares et qu'il faudrait mettre en valeur? Quelques-uns sont catalogués mais c'est loin d'être le cas de tous, comment faire? Et puis il y a des gravures dans des portefeuilles, des photographies anciennes ici et là. Comment savoir si elles ont quelque intérêt ? Dans un coin des magasins, des moisissures sont brusquement apparues et semblent contaminer peu à peu les ouvrages. Comment s'en débarrasser ? Qui prévenir ? Si vous vous posez parfois ces délicates questions, ce manuel est pour vous.

(Dominique Coq, responsable scientifique du volume)

Éclairé par des retours d'expériences de spécialistes, ce manuel réunit un ensemble raisonné d’informations de base, qui présente la marche à suivre pour appréhender, traiter, signaler et valoriser ces fonds patrimoniaux ; il permet d'en comprendre l'environnement juridique, administratif et documentaire.

Un document indispensable pour les professionnels peu formés dans le domaine, confrontés pour la première fois à une collection patrimoniale, ou qui s'y destinent, et désireux d'acquérir les rudiments nécessaires pour la gérer. 